\title{
Petrological and geochemical evolution of the Tolbachik volcanic massif, Kamchatka, Russia
}

\author{
Tatiana G. Churikova ${ }^{\mathrm{a}, *}$, Boris N. Gordeychik ${ }^{\mathrm{b}}$, Hikaru Iwamori ${ }^{\text {c,e }}$, Hitomi Nakamura ${ }^{\text {c,e }}$, Osamu Ishizuka ${ }^{\mathrm{d}}$, \\ Tatsuji Nishizawa $^{\mathrm{e}}$, Satoru Haraguchi ${ }^{\mathrm{c}}$, Takashi Miyazaki ${ }^{\mathrm{c}}$, Bogdan S. Vaglarov ${ }^{\mathrm{c}}$ \\ a Institute of Volcanology and Seismology, Far East Branch, Russian Academy of Sciences, 9 Piip Boulevard, Petropavlovsk-Kamchatsky, 683006, Russia \\ b Institute of Experimental Mineralogy, Russian Academy of Sciences, Academica Osypyana ul., 4, Chernogolovka, Moscow Region, 142432, Russia \\ ' Department of Solid Earth Geochemistry, Japan Agency for Marine-Earth Science and Technology, 2-15 Natsushima-cho, Yokosuka, Kanagawa 237-0061, Japan \\ d Research Institute of Earthquake and Volcano Geology, Geological Survey of Japan/AIST, Central 7, 1-1-1 Higashi, Tsukuba, Ibaraki 305-8567, Japan \\ e Department of Earth and Planetary Sciences, Tokyo Institute of Technology, 2-12-1 Ookayama, Meguro, Tokyo 152-8550, Japan
}

\section{A R T I C L E I N F O}

\section{Article history:}

Received 15 February 2015

Accepted 30 October 2015

Available online 6 November 2015

\section{Keywords:}

Central Kamchatka Depression

Klyuchevskaya group

Tolbachik volcanic massif

Subduction

Geochemistry

Fractional crystallization

\begin{abstract}
A B S T R A C T
Data on the geology, petrography, and geochemistry of Middle-Late-Pleistocene rocks from the Tolbachik volcanic massif (Kamchatka, Klyuchevskaya group of volcanoes) are presented and compared with rocks from the neighboring Mount Povorotnaya, Klyuchevskaya group basement, and Holocene-historical Tolbachik monogenetic cones. Two volcanic series of lavas, middle- $\mathrm{K}$ and high-K, are found in the Tolbachik massif. The results of our data analysis and computer modeling of crystallization at different $\mathrm{P}-\mathrm{T}-\mathrm{H}_{2} \mathrm{O}-\mathrm{fO}_{2}$ conditions allow us to reconstruct the geochemical history of the massif. The Tolbachik volcanic massif started to form earlier than $86 \mathrm{ka}$ based on K-Ar dating. During the formation of the pedestal and the lower parts of the stratovolcanoes, the middle-K melts, depleted relative to NMORB, fractionated in water-rich conditions (about $3 \%$ of $\mathrm{H}_{2} \mathrm{O}$ ). At the Late Pleistocene-Holocene boundary, a large fissure zone was initiated and the geodynamical regime changed. Upwelling associated with intra-arc rifting generated melting from the same mantle source that produced magmas more enriched in incompatible trace elements and subduction components; these magmas are high-K, not depleted relative to N-MORB melts with island arc signatures and rift-like characteristics. The fissure opening caused degassing during magma ascent, and the high-K melts fractionated at anhydrous conditions. These high-K rocks contributed to the formation of the upper parts of stratovolcanoes. At the beginning of Holocene, the high-K rocks became prevalent and formed cinder cones and associated lava fields along the fissure zone. However, some features, including 1975-1976 Northern Breakthrough, are represented by middle-K high-Mg rocks, suggesting that both middle-K and high-K melts still exist in the Tolbachik system. Our results show that fractional crystallization at different water conditions and a variably depleted upper mantle source are responsible for all observed variations in rocks within the Tolbachik volcanic massif. Sr-Nd isotopes are consistent with $2-4 \%$ crustal assimilation during formation of the pedestal and stratovolcanoes, while the young lava fields do not show evidence of crustal assimilation. Major and trace element data coupled with $\mathrm{K}-\mathrm{Ar}$ dating provide strong evidence that Mount Povorotnaya, located in $8 \mathrm{~km}$ northeast of Plosky Tolbachik, is an old block of the Tolbachik massif pedestal and for the moment it is the oldest (306 ka) known object in Klyuchevskaya group of volcanoes.
\end{abstract}

(c) 2015 Elsevier B.V. All rights reserved.

\section{Introduction}

Most Holocene volcanic activity on the Kamchatka peninsula has occurred within the Klyuchevskaya group of volcanoes (KGV), located in the northern part of the Central Kamchatka Depression (CKD). The tectonic setting of this area is dominated by a triple junction of lithospheric plates: Pacific, Bering, and Okhotsk. Presently, the origin of the

\footnotetext{
* Corresponding author.

E-mail address: tchurikova@mail.ru (T.G. Churikova).
}

geochemical diversity of the volcanic rocks in this area is the most discussed and critical one among the issues of magma origin at the Kamchatka subduction zone. Although a number of published papers have provided data on KGV volcanoes (Khrenov et al., 1991; Kersting and Arculus, 1994, 1995; Pineau et al., 1999; Dorendorf et al., 2000; Ozerov, 2000; Churikova et al., 2001, 2007, 2013; Mironov et al., 2001; Portnyagin et al., 2007a,b; Turner et al., 2007), most of them address only the products of recent and historical eruptions. And yet, it is impossible to understand the time-spatial evolution of the KGV without considering the pre-historical volcanism of each individual volcano and the 
entire area in question. Systematic studies of many KGV volcanoes were not conducted until recently and their geochemical and age correlation remains poorly constrained.

These unstudied volcanoes include not only such presently extinct volcanoes as Udina, Zimina, and Gorny Zub, but also Ostry Tolbachik and Plosky Tolbachik stratovolcanoes, on the slopes of which new monogenic cones were actively developing during the last $10 \mathrm{ka}$, erupting lavas of different compositions (Fig. 1). Numerous national and international publications were dedicated to Plosky Tolbachik volcano eruptions and adjacent monogenetic cones, which erupted repeatedly during the Holocene, including historical times (i.e. Vlodavets, 1937; Popkov, 1946; Piip, 1946, 1954; Menyailov, 1953; Sirin and Farberov, 1963; Kirsanov and Ponomarev, 1974; Ivanov and Khrenov, 1979; Fedotov and Markhinin, 1983; Fedotov, 1984; Krivenko, 1990; Kersting, 1995; Tatsumi et al., 1995; Hochstaedter et al., 1996; Kepezhinskas et al., 1997; Turner et al., 1998; Pineau et al., 1999; Volynets et al., 2000; Churikova et al., 2001; Münker et al., 2004; Portnyagin et al., 2007a; Volynets et al., 2013; see also Churikova et al., 2015). However, the referenced data mainly relate to monogenetic cones, whereas the information specifically on stratovolcanoes is practically nonexistent. Tolbachik massif was studied on a reconnaissance basis only in the 1970s. During that time, a geological map of this volcano was developed and the first petrographical and mineralogical data were obtained from its rocks (Ermakov, 1977). Presently, only a few papers have been published on the geology and petrography of Ostry and Plosky Tolbachik stratovolcanoes (Ermakov and Vazheevskaya, 1973) together with a few papers addressing to a limited extent the petrochemistry of the rocks, and mineral compositions (Ermakov, 1977; Flerov and Melekestsev, 2013; Flerov et al., 2015). Even though the data published in the 20th century showed that the cinder cones of the Tolbachik volcanic massif erupted distinct magmas ranging from high-Mg basalts to high-K trachybasalts, modern geochemical and isotope studies of the stratovolcanoes were not conducted, therefore, the evolution of those rocks was not clearly understood.

To determine the rock variations within the Tolbachik volcanic massif and to investigate the petrogenetic relationships between the rocks of Plosky and Ostry Tolbachik stratovolcanoes, dike complex, lavas of the adjacent zone of lava fields, and adjacent centers of the KGV, in this paper we present geological, petrographical, petrochemical, geochemical, isotopic and some $\mathrm{K}-\mathrm{Ar}$ data on the rocks of Tolbachik volcanic massif. The present paper is based on the study of a representative collection of 155 samples from stratovolcanoes, dikes and monogenetic cones of different ages, including the 2012-2013 eruption (Fig. 2). Additionally, our study included samples from the separate edifice of Mount Povorotnaya and the KGV basement located
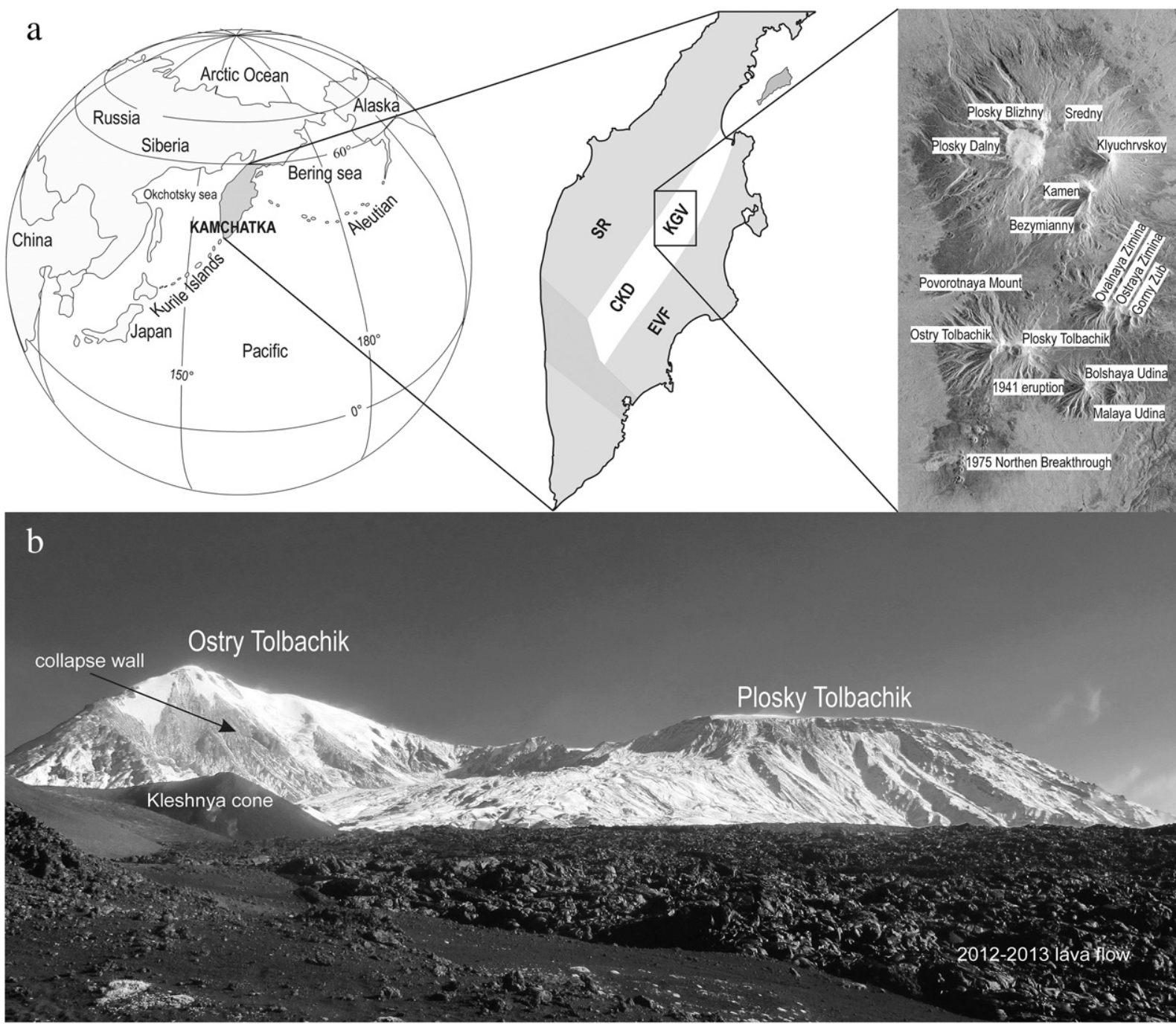

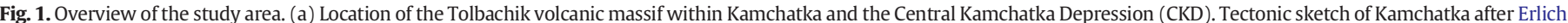

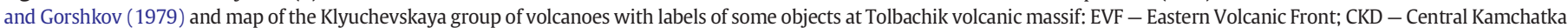

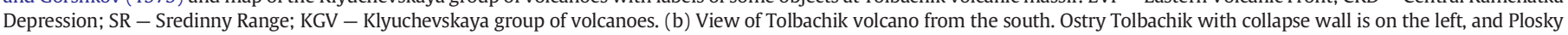
Tolbachik is on the right. The lava flow of 2012-2013 eruption is in the foreground. 


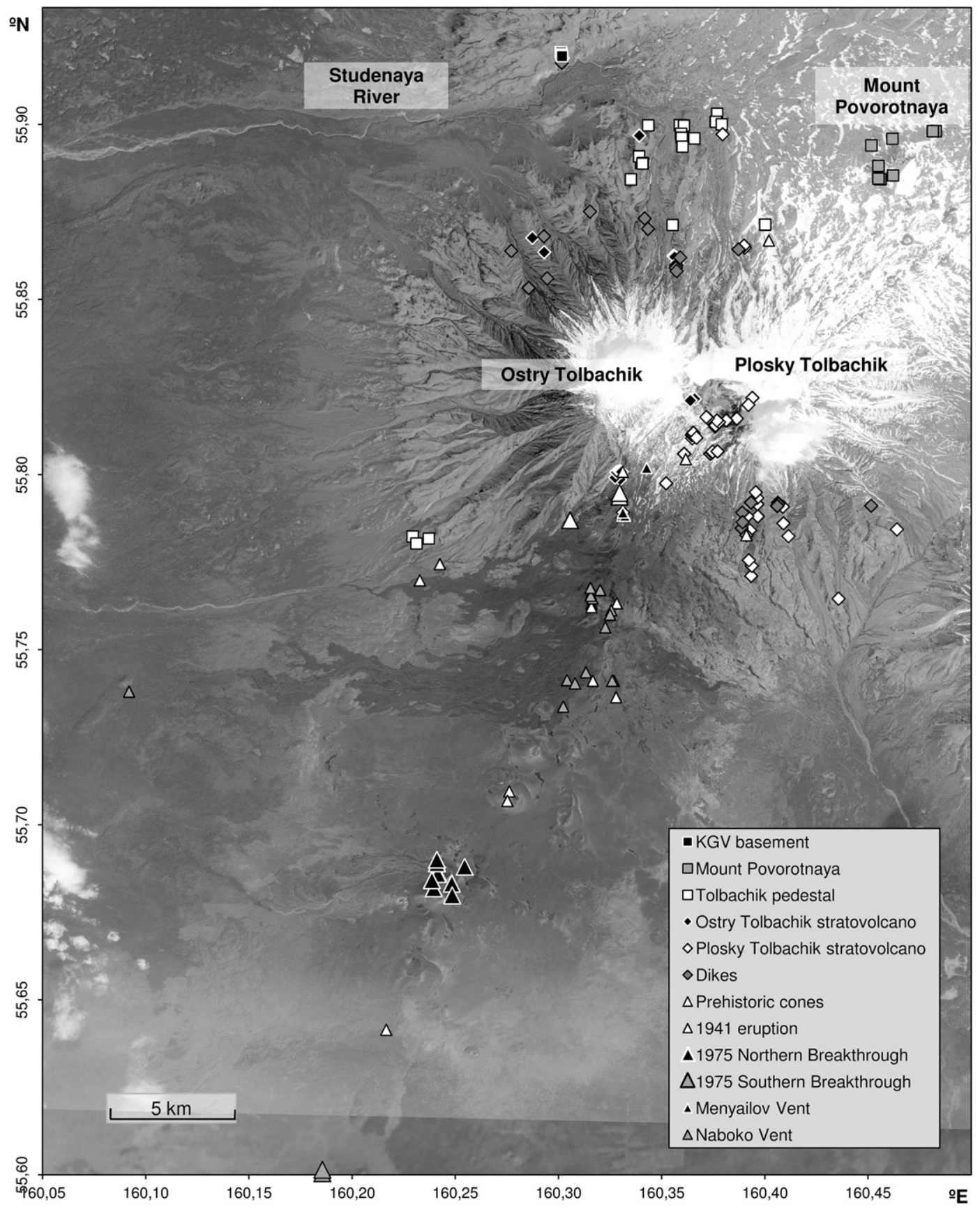

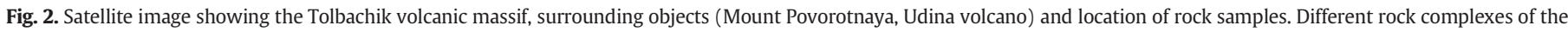

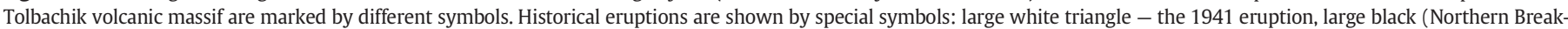

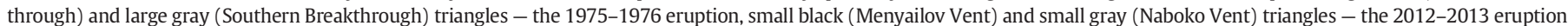

just to the north of Tolbachik massif. Major and trace element whole rock data show that consistent differences exist in rocks of different ages.

\section{Geology of the Tolbachik volcanic massif}

The KGV basement centered underneath Ushkovsky volcano (Flerov and Ovsyannikov, 1991) with the age based on Ar-Ar dating at 262-274 ka (Calkins, 2004) was formed in Early- to MiddlePleistocene times and was divided by a series of faults into several tectonic blocks in Late-Pleistocene (Melekestsev et al., 1974). The Tolbachik volcanic massif is located on the tectonically unstable southwestern block, which shifted down relative to the tectonically stable northern block underlying the Klyuchevskoy, Kamen,
Ushkovsky, Krestovsky, Bezymianny and Sredny volcanoes and the southeastern blocks by about $500 \mathrm{~m}$. The Tolbachik volcanic massif and neighboring volcanoes Bolshaya Udina, Malaya Udina, and, probably, Ovalnaya Zimina, Ostraya Zimina, and Gorny Zub are situated above the bounding faults (Timerbaeva, 1967; Melekestsev et al., 1974).

The Tolbachik volcanic massif is located in the southwestern part of KGV (Fig. 1). It occupies a large area extending from the Studenaya River in the north to the Tolbachik River in the south. According to previous geological mapping (Ermakov and Vazheevskaya, 1973) and based on our study (Fig. 2), the geological history of the Tolbachik massif consists of four consecutive periods of volcanic activity: (1) pedestal formation; (2) nearly simultaneous growth of two stratovolcanoes; (3) development 
of a dike complex; and (4) formation of numerous cinder cones and monogenetic lava fields.

Substantially forest-covered and eroded, the pedestal of the volcano (period 1), which has a diameter of more than $20 \mathrm{~km}$ and an inclination angle not exceeding $5^{\circ}$, is exposed in northern, northwestern, and southwestern sectors of the Tolbachik volcanic massif, and is represented by single blocks, outliers, necks, and spurs. At present, the pedestal is partially split by deep glacial valleys on the northern and western

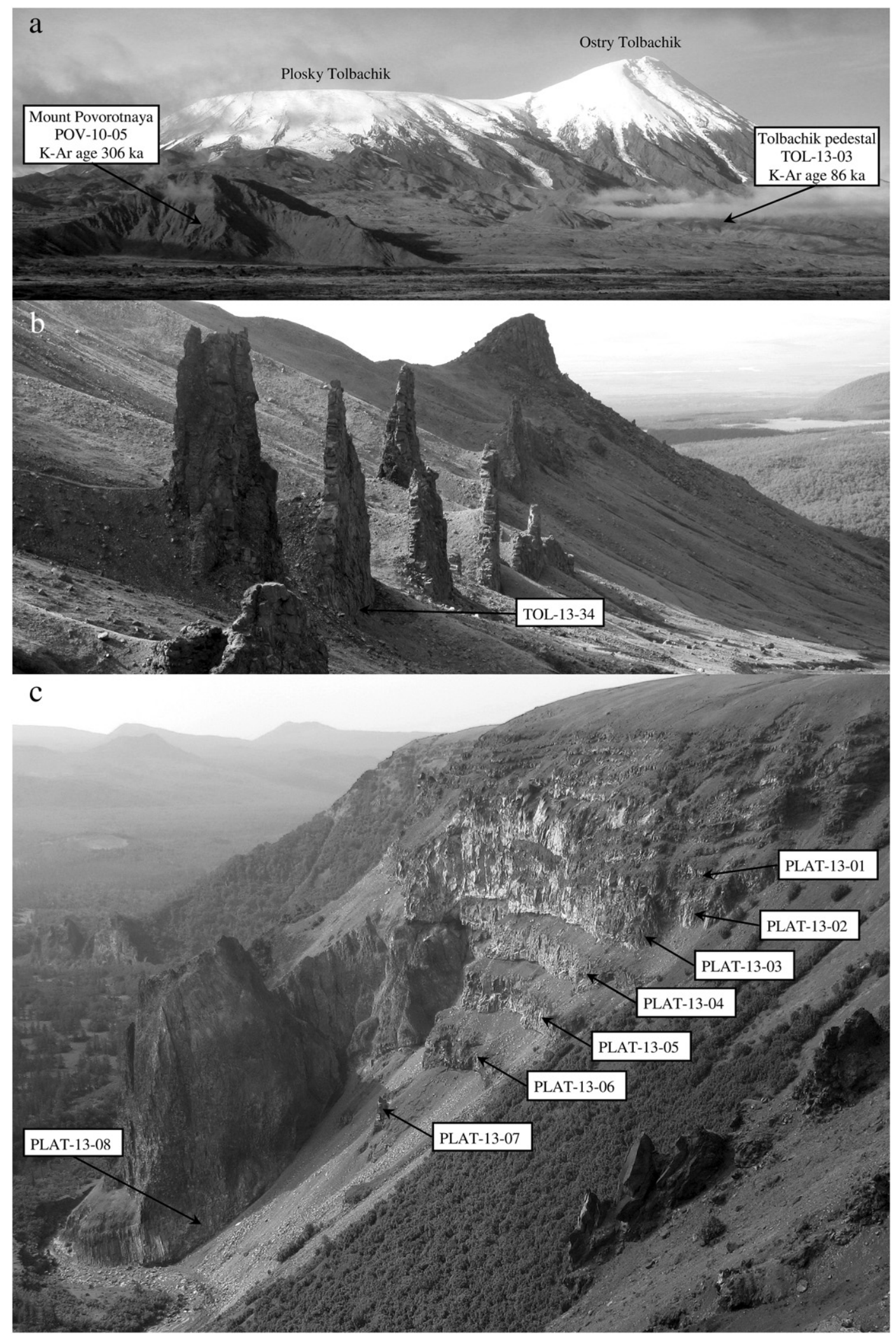

Fig. 3. Plate of field images showing features of different complexes of the Tolbachik volcanic massif. (a) View of Tolbachik volcano and Mount Povorotnaya from the north. Sample location of rocks that were dated by K-Ar method shown by errors. (b) Large continuous dike at the NW slope of the Ostry Tolbachik volcano. (c) KGV basement outcrop at the Studenaya River valley. The sample PLAT-13-08 is the neck nearby this outcrop. The level difference between samples PLAT-13-01 and PLAT-13-08 is $225 \mathrm{~m}$ (e-Table 1). 
slopes, and is mainly covered by volcanic products (lavas and cinder) of subsequent eruptions, as well as by the deposits of edifice collapses at the southern, eastern and southeastern slopes. So-called augite-phyric rocks (after Piip, 1954, who first described these rare rocks), with up to $30 \%$ augite as the only phenocryst, are the main mineralogical feature of the pedestal complex, and such rocks were not observed in any other complexes of the Tolbachik massif.

Ostry and Plosky Tolbachik stratovolcanoes (period 2) differ from the pedestal morphologically as they have higher, up to $20-30^{\circ}$, angles of inclination (Figs. 1b, 3a). According to geological observations, both stratovolcanoes were developing simultaneously (Ermakov and Vazheevskaya, 1973; Flerov et al., 2015). Ostry Tolbachik volcano is extinct now, while the summit eruptions at Plosky Tolbachik volcano have been repeatedly occurring during the Holocene and historical time, in some instances simultaneously with flank fissure eruptions, in others, without (see also Churikova et al., 2015).

Similar to other large volcanic edifices in the KGV (e.g. Khrenov et al., 1991; Melekestsev et al., 1991; Churikova et al., 2013), the inner parts of the stratovolcanoes comprise thick pyroclastic deposits, which is consistent with mainly explosive eruptions during the first periods of the development. The later periods were dominated by extensive lava effusion. The thickness of the lava flows is normally less than 5-7 m and in some instances is only $0.5-1 \mathrm{~m}$. Such thin lavas could flow down the slopes of the volcano to a distance of several kilometers, consistent with a very low viscosity. The upper parts of both edifices are covered by thick glaciers that descend in multiple ice streams into the majority of the flank valleys.

Macroscopically, stratovolcano rocks are represented by massive or flagstone lavas with porosities that vary from dense to cinder-like varieties enriched with various phenocrysts. Textures of the rocks vary from subaphyric to serioporphyritic and megaporphyritic. The main rock forming minerals are olivine $(\mathrm{Ol})$, clinopyroxene $(\mathrm{Cpx})$, plagioclase $(\mathrm{Pl})$, magnetite (Mt), and, to a lesser extent, orthopyroxene (Opx).

A dike complex (period 3) was developed during pedestal and stratovolcano formation. The numerous dikes that crosscut the older edifices form a radial pattern as well as ring structures in all sectors of both edifices. Their widths range from 1 to $10 \mathrm{~m}$ and the lengths reach up to $2 \mathrm{~km}$ (Fig. 3b). Dike petrology varies from rocks similar to those of the stratovolcanoes, Ol-Px bearing lavas with 25-30\% mafic minerals on the NE flank, to megaplagiophyric and fine-banded samples on the SSE sides. The large variations in chemical composition of dikes suggest several periods of intrusions.

Subsequent geological processes, including the development of a fissure eruption zone that crosses the top of Plosky Tolbachik volcano, led to the destruction of the Plosky Tolbachik summit resulting in formation of a $3 \mathrm{~km}$ diameter Hawaiian-type pit crater/caldera. Ostry Tolbachik stratovolcano was impacted by a series of landslides (Ponomareva et al., 2006), with the largest directed to SE (Figs. 1, 2).

The final and currently ongoing stage of the Tolbachik massif activity (period 4) started about $10 \mathrm{ka}$ ago with the formation of monogenetic cones and lava flows along a S-SW to NE fissure system. The cones are composed mainly of scoria, and frequently are the vents to lava flows as long as $10 \mathrm{~km}$. During the period dating $10-2 \mathrm{ka}$, only subalkaline, high-K, mainly megaplagiophyric (with Pl phenocrysts up to $2 \mathrm{~cm}$ ) basalts were erupted. But starting c. 2 ka eruptions of high-Mg basalts began as the high-K eruptions continued (Flerov and Melekestsev, 2013; Flerov et al., 2015). During the 1975-1976 eruption four types of basalts were observed: high-Mg, high-K (and high-Al) and two intermediate types (Flerov et al., 1984). The high-Mg lavas occurred only during the first two months of the Northern Breakthrough and are referred to as "High-Mg basalts of 1975-1976 Northern Breakthrough" throughout this paper.

We sampled lavas in N-NW and SW-S-SE sectors of the Tolbachik volcanic massif to insure complete coverage. The NE and SW sectors, even though densely covered by products of recent eruptions and the older outcrops, are not exposed at the surface and sampling of the pedestal rocks in these sectors is either very difficult or impossible. For comparison, we also sampled Mount Povorotnaya, situated $8 \mathrm{~km}$ to NE of the Plosky Tolbachik caldera (Fig. 3a), and the old plateau lavas underlying the KGV, which are located to the north of the Tolbachik volcanic massif with the outcrops in Studenaya River valley (Fig. 3c). All samples included in this study were taken from natural outcrops, are unaltered samples, and represent lavas, volcanic bombs and cinderlike blocks.

Modern geochemical studies have only been completed on the youngest Holocene complex (4); periods 1-3 have not been geochemically characterized and their interrelation and petrogenesis, as opposed to the cinder cone lavas and other KGV volcanoes, are unknown. Furthermore, the formation of the pedestal and stratovolcanoes of the Tolbachik volcanic massif is a blank page of the pre-Holocene KGV history.

\section{Analytical techniques}

Major elements of whole rock samples were analyzed at University of Tokyo (Japan) (Table 1 and e-Table 1). Major elements for 155 samples, including 8 lavas of Mount Povorotnaya and 6 samples from the KGV basaltic andesite plateau to the north of the Tolbachik massif were determined by standard XRF analysis on glass disks, prepared as a mixture of $400 \mathrm{mg}$ whole rock powders with a lithium tetraborate flux. Based on repeat analyses of the standards JA-2, JB-2 and JB-3, analytical errors for $\mathrm{SiO}_{2}, \mathrm{Al}_{2} \mathrm{O}_{3}, \mathrm{CaO}$ and $\mathrm{Na}_{2} \mathrm{O}$ are better than $1 \%$, and are within $2 \%$ for other elements.

Trace elements for 102 samples were analyzed by inductively coupled plasma mass spectrometry (ICPMS) at the Tokyo Institute of Technology (TITECH, Japan) (Table 1 and e-Table 1). About $50 \mathrm{mg}$ whole rock powders was dissolved in Teflon beakers with a mixture of $\mathrm{HF}$ and $\mathrm{HClO}_{4}$, and, following evaporation, was redissolved in $\mathrm{HNO}_{3}$ for the measurement. The international standards JA-1, JB-2 and JB-3 were analyzed repeatedly together with samples to check the reproducibility. From this we estimate the error for Cs, Sr, Nd, Zr, Hf, Sm, Eu, Gd, $\mathrm{Tb}, \mathrm{Er}, \mathrm{Cu}, \mathrm{La}, \mathrm{Ce}$ to be around $10 \%$, for all other trace elements the error is around $20 \%$, with $\mathrm{Nb}$ and Ta yielding only semi-quantitative results.

Strontium and Nd isotope analyses were conducted at D-SEG JAMSTEC. The analytical procedure used for the chemical separation and mass spectrometry for $\mathrm{Sr}$ and $\mathrm{Nd}$ isotope determinations is outlined in Hirahara et al. (2009), Takahashi et al. (2009), and Miyazaki et al. (2012). Total procedural blanks for $\mathrm{Sr}$ and Nd during the measurement period were less than $177 \mathrm{pg}$ and $5 \mathrm{pg}$, respectively. Sr and $\mathrm{Nd}$ isotope ratios were measured by thermal ionization mass spectrometry (TIMS: Triton; Thermo-Finnigan). The $\mathrm{Sr}$ and Nd isotope ratios were normalized to ${ }^{86} \mathrm{Sr} /{ }^{88} \mathrm{Sr}=0.1194$ and ${ }^{146} \mathrm{Nd} /{ }^{144} \mathrm{Nd}=0.7219$, respectively, to correct for mass fractionation. The mean ${ }^{87} \mathrm{Sr} /{ }^{86} \mathrm{Sr}$ value in the NIST 987 standard was $0.710220( \pm 0.000012 ; 2 \sigma, \mathrm{n}=13)$ and the mean ${ }^{143} \mathrm{Nd} /{ }^{144} \mathrm{Nd}$ value in the JNdi-1 standard was 0.512098 $( \pm 0.000013 ; 2 \sigma, \mathrm{n}=15)$.

Potassium-Ar dating (Table 2) was carried out at the Institute of Geology and Geoinformation (Tsukuba, Japan). For sample preparation chips of c. $50 \mathrm{~g}$ weight were taken from the unaltered and wellcrystallized parts of a sample with a water-cooled saw. The chips were crushed to 250-500 $\mu \mathrm{m}$ using an Fe pestle. Crushed samples were flushed with deionized water in an ultrasonic bath. Some phenocrysts such as olivine and pyroxene might host extraneous Ar derived from mantle that has higher ${ }^{40} \mathrm{Ar} /{ }^{36} \mathrm{Ar}$ ratios compared to atmospheric $\mathrm{Ar}$. Inclusion of these phenocrysts potentially gives erroneously older ages. To avoid this possibility, large phenocrysts (size $>0.5 \mathrm{~mm}$ ) were removed prior to the analysis by using: (1) a magnetic separator; (2) heavy liquids; and then (3) hand-picking under a binocular microscope. For $\mathrm{K}$ analysis, the 3-5 $\mathrm{g}$ samples for $\mathrm{Ar}$ isotope analysis were further pulverized using an agate mortar.

Argon isotopic ratios were determined by a conventional isotope dilution method using a ${ }^{38} \mathrm{Ar}$ spike. Argon extraction from samples was 
Table 1

Major and trace element abundances in representative set of the volcanic rocks of Tolbachik massif, Mount Povorotnaya and KGV basement.

\begin{tabular}{|c|c|c|c|c|c|c|}
\hline Sample & TOL-12-01 & TOL-12-02 & TOL-12-18 & TOL-12-15 & TOL-12-21 & TOL-12-37 \\
\hline Complex & PT strato & PT strato & PT strato & PT strato & PT strato & PT strato \\
\hline Trend & 2 & 2 & 2 & 2 & 2 & 1 \\
\hline Lat (N) & $55^{\circ} 49^{\prime} 19^{\prime \prime}$ & $55^{\circ} 49^{\prime} 11.7^{\prime \prime}$ & $55^{\circ} 48^{\prime} 58^{\prime \prime}$ & $55^{\circ} 48^{\prime} 54.1^{\prime \prime}$ & $55^{\circ} 48^{\prime} 41.6^{\prime \prime}$ & $55^{\circ} 46^{\prime} 32^{\prime \prime}$ \\
\hline Long (E) & $160^{\circ} 23^{\prime} 38^{\prime \prime}$ & $160^{\circ} 23^{\prime} 31.2^{\prime \prime}$ & $160^{\circ} 22^{\prime} 20.5^{\prime \prime}$ & $160^{\circ} 22^{\prime} 39.8^{\prime \prime}$ & $160^{\circ} 21^{\prime} 53.6^{\prime \prime}$ & $160^{\circ} 23^{\prime} 32^{\prime \prime}$ \\
\hline $\mathrm{SiO}_{2}$ & 51.38 & 51.81 & 51.59 & 51.96 & 50.42 & 53.00 \\
\hline $\mathrm{TiO}_{2}$ & 1.29 & 1.36 & 1.64 & 1.59 & 1.62 & 1.16 \\
\hline $\mathrm{Al}_{2} \mathrm{O}_{3}$ & 16.85 & 17.51 & 16.36 & 17.64 & 18.19 & 17.33 \\
\hline $\mathrm{FeO}$ & 10.22 & 9.60 & 10.44 & 9.88 & 10.17 & 9.35 \\
\hline $\mathrm{MnO}$ & 0.16 & 0.14 & 0.17 & 0.14 & 0.16 & 0.16 \\
\hline $\mathrm{MgO}$ & 5.63 & 4.90 & 5.39 & 4.44 & 5.23 & 5.21 \\
\hline $\mathrm{CaO}$ & 9.65 & 9.39 & 9.00 & 8.57 & 8.78 & 9.10 \\
\hline $\mathrm{Na}_{2} \mathrm{O}$ & 3.01 & 3.29 & 3.08 & 3.28 & 3.10 & 3.09 \\
\hline $\mathrm{K}_{2} \mathrm{O}$ & 1.41 & 1.57 & 1.79 & 1.92 & 1.79 & 1.32 \\
\hline $\mathrm{P}_{2} \mathrm{O}_{5}$ & 0.40 & 0.43 & 0.54 & 0.58 & 0.54 & 0.28 \\
\hline $\mathrm{Cr}$ & 163 & 113 & 109 & 73 & 92 & 62 \\
\hline $\mathrm{Ni}$ & 55 & 47 & 39 & 33 & 38 & 21 \\
\hline $\mathrm{Cu}$ & 159 & 170 & 176 & 159 & 133 & 69 \\
\hline $\mathrm{Zn}$ & 90 & 87 & 100 & 99 & 106 & 86 \\
\hline $\mathrm{Rb}$ & 39.33 & 42.23 & 53.18 & 55.59 & 56.63 & 26.32 \\
\hline $\mathrm{Sr}$ & 397 & 383 & 367 & 367 & 385 & 416 \\
\hline $\mathrm{Y}$ & 30.11 & 29.98 & 38.35 & 37.17 & 39.97 & 27.51 \\
\hline $\mathrm{Zr}$ & 173 & 188 & 234 & 243 & 246 & 114 \\
\hline $\mathrm{Nb}$ & 4.35 & 4.78 & 5.96 & 6.29 & 5.50 & 3.06 \\
\hline $\mathrm{Cd}$ & 0.12 & 0.13 & 0.15 & 0.15 & 0.15 & 0.10 \\
\hline Sn & 1.37 & 1.24 & 1.32 & 1.28 & 1.77 & 0.69 \\
\hline Cs & 1.12 & 1.32 & 1.50 & 1.61 & 1.48 & 0.75 \\
\hline $\mathrm{Ba}$ & 384 & 456 & 434 & 476 & 435 & 483 \\
\hline $\mathrm{La}$ & 13.75 & 14.77 & 16.38 & 16.90 & 16.58 & 10.58 \\
\hline $\mathrm{Ce}$ & 33.66 & 36.00 & 40.62 & 41.52 & 40.94 & 25.56 \\
\hline $\mathrm{Pr}$ & 4.89 & 5.16 & 5.73 & 5.84 & 5.78 & 3.75 \\
\hline $\mathrm{Nd}$ & 23.67 & 24.63 & 27.48 & 27.87 & 27.60 & 18.52 \\
\hline $\mathrm{Sm}$ & 5.94 & 6.14 & 6.90 & 6.94 & 6.93 & 4.98 \\
\hline $\mathrm{Eu}$ & 1.75 & 1.81 & 1.93 & 1.95 & 1.93 & 1.56 \\
\hline $\mathrm{Gd}$ & 6.25 & 6.43 & 7.30 & 7.23 & 7.31 & 5.35 \\
\hline $\mathrm{Tb}$ & 0.93 & 0.96 & 1.10 & 1.08 & 1.10 & 0.81 \\
\hline Dy & 5.66 & 5.80 & 6.77 & 6.55 & 6.74 & 4.98 \\
\hline Ho & 0.99 & 1.02 & 1.19 & 1.15 & 1.19 & 0.87 \\
\hline $\mathrm{Er}$ & 3.13 & 3.21 & 3.78 & 3.63 & 3.77 & 2.73 \\
\hline $\mathrm{Tm}$ & 0.53 & 0.55 & 0.64 & 0.61 & 0.64 & 0.46 \\
\hline $\mathrm{Yb}$ & 3.30 & 3.39 & 3.96 & 3.80 & 3.96 & 2.83 \\
\hline $\mathrm{Lu}$ & 0.51 & 0.52 & 0.61 & 0.59 & 0.61 & 0.43 \\
\hline $\mathrm{Hf}$ & 4.05 & 4.42 & 4.91 & 5.14 & 4.92 & 3.13 \\
\hline Та & 0.31 & 0.34 & 0.39 & 0.42 & 0.32 & 0.20 \\
\hline $\mathrm{Pb}$ & 4.71 & 5.38 & 5.07 & 5.65 & 5.26 & 3.91 \\
\hline Th & 1.56 & 1.75 & 1.80 & 2.02 & 1.85 & 0.94 \\
\hline $\mathrm{U}$ & 1.00 & 1.12 & 1.16 & 1.27 & 1.18 & 0.63 \\
\hline Sample & TOL-12-38 & TOL-12-41 & TOL-12-42 & TOL-12-46 & TOL-12-53 & TOL-13-42 \\
\hline Complex & PT strato & PT strato & PT strato & PT strato & PT strato & PT strato \\
\hline Trend & 2 & 1 & 1 & 2 & 1 & 1 \\
\hline Lat (N) & $55^{\circ} 47^{\prime} 2.8^{\prime \prime}$ & $55^{\circ} 47^{\prime} 36.1^{\prime \prime}$ & $55^{\circ} 47^{\prime} 41.9^{\prime \prime}$ & $55^{\circ} 46^{\prime} 56.7^{\prime \prime}$ & $55^{\circ} 45^{\prime} 52.5^{\prime \prime}$ & $55^{\circ} 51^{\prime} 55.4^{\prime \prime}$ \\
\hline Long (E) & $160^{\circ} 23^{\prime} 33.8^{\prime \prime}$ & $160^{\circ} 23^{\prime} 46.5^{\prime \prime}$ & $160^{\circ} 23^{\prime} 44^{\prime \prime}$ & $160^{\circ} 24^{\prime} 41^{\prime \prime}$ & $160^{\circ} 26^{\prime} 8.5^{\prime \prime}$ & $160^{\circ} 23^{\prime} 23.5^{\prime \prime}$ \\
\hline $\mathrm{SiO}_{2}$ & 51.16 & 50.15 & 50.57 & 51.60 & 56.72 & 50.60 \\
\hline $\mathrm{TiO}_{2}$ & 1.51 & 1.21 & 1.16 & 1.61 & 0.97 & 1.17 \\
\hline $\mathrm{Al}_{2} \mathrm{O}_{3}$ & 16.71 & 19.22 & 17.78 & 16.94 & 19.95 & 16.77 \\
\hline $\mathrm{FeO}$ & 11.14 & 10.16 & 10.51 & 10.13 & 6.58 & 10.00 \\
\hline $\mathrm{MnO}$ & 0.18 & 0.17 & 0.18 & 0.16 & 0.12 & 0.21 \\
\hline $\mathrm{MgO}$ & 5.39 & 4.57 & 5.43 & 4.87 & 2.15 & 6.97 \\
\hline $\mathrm{CaO}$ & 8.88 & 10.03 & 10.00 & 8.86 & 7.07 & 10.22 \\
\hline $\mathrm{Na}_{2} \mathrm{O}$ & 3.09 & 3.07 & 2.94 & 3.38 & 4.24 & 2.87 \\
\hline $\mathrm{K}_{2} \mathrm{O}$ & 1.54 & 1.13 & 1.16 & 1.91 & 1.83 & 0.97 \\
\hline $\mathrm{P}_{2} \mathrm{O}_{5}$ & 0.40 & 0.28 & 0.28 & 0.54 & 0.37 & 0.23 \\
\hline $\mathrm{Cr}$ & 94 & 18 & 39 & 92 & 10 & 146 \\
\hline $\mathrm{Ni}$ & 44 & 18 & 23 & 37 & 7 & 54 \\
\hline $\mathrm{Cu}$ & 163 & 108 & 96 & 212 & 40 & 55 \\
\hline $\mathrm{Zn}$ & 103 & 94 & 88 & 104 & 70 & 85 \\
\hline $\mathrm{Rb}$ & 38.46 & 22.85 & 22.57 & 57.54 & 30.47 & 14.53 \\
\hline $\mathrm{Sr}$ & 391 & 503 & 432 & 419 & 416 & 358 \\
\hline $\mathrm{Y}$ & 38.36 & 28.63 & 27.43 & 41.03 & 29.62 & 21.37 \\
\hline $\mathrm{Zr}$ & 195 & 95 & 94 & 257 & 186 & 81 \\
\hline $\mathrm{Nb}$ & 4.66 & 2.53 & 2.39 & 6.18 & 4.02 & 2.14 \\
\hline $\mathrm{Cd}$ & 0.14 & 0.10 & 0.10 & 0.15 & 0.11 & 0.08 \\
\hline Sn & 0.83 & 0.69 & 0.59 & 1.09 & 0.45 & 0.48 \\
\hline
\end{tabular}


Table 1 (continued)

\begin{tabular}{|c|c|c|c|c|c|c|}
\hline Sample & TOL-12-38 & TOL-12-41 & TOL-12-42 & TOL-12-46 & TOL-12-53 & TOL-13-42 \\
\hline Complex & PT strato & PT strato & PT strato & PT strato & PT strato & PT strato \\
\hline Cs & 1.04 & 0.52 & 0.54 & 1.48 & 0.53 & 0.36 \\
\hline $\mathrm{Ba}$ & 497 & 331 & 359 & 508 & 518 & 264 \\
\hline $\mathrm{La}$ & 14.25 & 8.95 & 9.23 & 18.09 & 11.57 & 6.53 \\
\hline $\mathrm{Ce}$ & 35.36 & 22.44 & 22.88 & 44.09 & 28.45 & 17.16 \\
\hline $\operatorname{Pr}$ & 5.12 & 3.36 & 3.41 & 6.16 & 4.02 & 2.73 \\
\hline $\mathrm{Nd}$ & 25.27 & 17.11 & 17.16 & 29.29 & 19.35 & 14.11 \\
\hline $\mathrm{Sm}$ & 6.63 & 4.72 & 4.71 & 7.31 & 4.93 & 3.83 \\
\hline $\mathrm{Eu}$ & 1.93 & 1.55 & 1.51 & 2.07 & 1.54 & 1.28 \\
\hline Gd & 7.23 & 5.13 & 5.08 & 7.73 & 5.22 & 4.35 \\
\hline $\mathrm{Tb}$ & 1.09 & 0.78 & 0.78 & 1.13 & 0.79 & 0.68 \\
\hline Dy & 6.67 & 4.88 & 4.82 & 6.81 & 4.85 & 4.34 \\
\hline Ho & 1.17 & 0.85 & 0.85 & 1.19 & 0.86 & 0.89 \\
\hline $\mathrm{Er}$ & 3.71 & 2.68 & 2.66 & 3.76 & 2.79 & 2.54 \\
\hline $\mathrm{Tm}$ & 0.62 & 0.45 & 0.45 & 0.63 & 0.48 & 0.36 \\
\hline $\mathrm{Yb}$ & 3.87 & 2.76 & 2.75 & 3.92 & 2.98 & 2.36 \\
\hline $\mathrm{Lu}$ & 0.59 & 0.42 & 0.42 & 0.60 & 0.47 & 0.36 \\
\hline $\mathrm{Hf}$ & 4.21 & 2.56 & 2.62 & 4.95 & 3.73 & 2.23 \\
\hline Ta & 0.30 & 0.15 & 0.15 & 0.33 & 0.24 & 0.15 \\
\hline $\mathrm{Pb}$ & 4.79 & 2.76 & 3.15 & 5.59 & 4.56 & 1.81 \\
\hline Th & 1.40 & 0.68 & 0.73 & 1.94 & 1.27 & 0.60 \\
\hline $\mathrm{U}$ & 0.88 & 0.46 & 0.49 & 1.22 & 0.78 & 0.38 \\
\hline Sample & TOL-12-52 & TOL-12-61 & TOL-12-63 & TOL-12-64 & TOL-12-66 & TOL-13-13 \\
\hline$\overline{\text { Complex }}$ & PT strato & OT strato & OT strato & OT strato & OT strato & OT strato \\
\hline Trend & 1 & 2 & 2 & 2 & 2 & 2 \\
\hline Lat $(\mathrm{N})$ & $55^{\circ} 47^{\prime} 9.9^{\prime \prime}$ & $55^{\circ} 47^{\prime} 57^{\prime \prime}$ & $55^{\circ} 48^{\prime} 3.8^{\prime \prime}$ & $55^{\circ} 48^{\prime} 3.1^{\prime \prime}$ & $55^{\circ} 48^{\prime} 2.8^{\prime \prime}$ & $55^{\circ} 53^{\prime} 48.5^{\prime \prime}$ \\
\hline Long (E) & $160^{\circ} 24^{\prime} 32.4^{\prime \prime}$ & $160^{\circ} 19^{\prime} 38.6^{\prime \prime}$ & $160^{\circ} 19^{\prime} 45.8^{\prime \prime}$ & $160^{\circ} 19^{\prime} 48^{\prime \prime}$ & $160^{\circ} 19^{\prime} 50.1^{\prime \prime}$ & $160^{\circ} 20^{\prime} 20.8^{\prime \prime}$ \\
\hline $\mathrm{SiO}_{2}$ & 55.69 & 52.14 & 52.45 & 52.49 & 52.14 & 51.69 \\
\hline $\mathrm{TiO}_{2}$ & 1.00 & 1.47 & 1.54 & 1.57 & 1.47 & 1.84 \\
\hline $\mathrm{Al}_{2} \mathrm{O}_{3}$ & 18.08 & 16.93 & 17.82 & 17.99 & 16.98 & 16.72 \\
\hline $\mathrm{FeO}$ & 8.48 & 9.94 & 9.18 & 9.21 & 9.76 & 10.24 \\
\hline $\mathrm{MnO}$ & 0.14 & 0.15 & 0.14 & 0.16 & 0.14 & 0.18 \\
\hline $\mathrm{MgO}$ & 3.72 & 4.90 & 4.27 & 4.03 & 5.02 & 4.63 \\
\hline $\mathrm{CaO}$ & 7.46 & 8.68 & 8.47 & 8.31 & 8.70 & 8.38 \\
\hline $\mathrm{Na}_{2} \mathrm{O}$ & 3.68 & 3.28 & 3.54 & 3.63 & 3.28 & 3.40 \\
\hline $\mathrm{K}_{2} \mathrm{O}$ & 1.46 & 1.93 & 1.99 & 2.03 & 1.93 & 2.25 \\
\hline $\mathrm{P}_{2} \mathrm{O}_{5}$ & 0.29 & 0.58 & 0.59 & 0.59 & 0.58 & 0.68 \\
\hline $\mathrm{Cr}$ & 28 & 78 & 37 & 22 & 69 & 77 \\
\hline $\mathrm{Ni}$ & 18 & 40 & 33 & 25 & 37 & 35 \\
\hline $\mathrm{Cu}$ & 83 & 274 & 176 & 209 & 226 & 233 \\
\hline $\mathrm{Zn}$ & 97 & 104 & 93 & 98 & 95 & 122 \\
\hline $\mathrm{Rb}$ & 32.63 & 65.48 & 57.35 & 60.63 & 60.34 & 52.42 \\
\hline $\mathrm{Sr}$ & 450 & 399 & 369 & 382 & 374 & 371 \\
\hline $\mathrm{Y}$ & 29.47 & 39.28 & 37.10 & 38.75 & 36.81 & 34.68 \\
\hline $\mathrm{Zr}$ & 155 & 273 & 253 & 273 & 256 & 238 \\
\hline $\mathrm{Nb}$ & 3.58 & 4.50 & 5.10 & 6.10 & 4.63 & 5.71 \\
\hline $\mathrm{Cd}$ & 0.12 & 0.15 & 0.16 & 0.17 & 0.15 & 0.15 \\
\hline Sn & 0.66 & 0.97 & 0.91 & 0.79 & 0.95 & 0.85 \\
\hline Cs & 0.59 & 1.81 & 0.96 & 1.82 & 1.85 & 1.66 \\
\hline $\mathrm{Ba}$ & 558 & 507 & 516 & 542 & 530 & 524 \\
\hline $\mathrm{La}$ & 12.39 & 19.12 & 18.71 & 19.76 & 19.60 & 19.61 \\
\hline $\mathrm{Ce}$ & 28.92 & 45.76 & 45.00 & 47.38 & 47.18 & 46.66 \\
\hline $\mathrm{Pr}$ & 4.07 & 6.25 & 6.18 & 6.46 & 6.42 & 7.29 \\
\hline $\mathrm{Nd}$ & 19.36 & 29.23 & 28.91 & 30.16 & 30.12 & 34.43 \\
\hline $\mathrm{Sm}$ & 4.90 & 7.09 & 7.03 & 7.32 & 7.33 & 7.89 \\
\hline $\mathrm{Eu}$ & 1.55 & 1.93 & 1.99 & 2.07 & 2.00 & 2.12 \\
\hline Gd & 5.21 & 7.32 & 7.46 & 7.81 & 7.58 & 8.40 \\
\hline $\mathrm{Tb}$ & 0.78 & 1.06 & 1.09 & 1.13 & 1.10 & 1.20 \\
\hline Dy & 4.81 & 6.34 & 6.56 & 6.80 & 6.53 & 7.16 \\
\hline Ho & 0.85 & 1.11 & 1.15 & 1.19 & 1.13 & 1.44 \\
\hline $\mathrm{Er}$ & 2.73 & 3.50 & 3.63 & 3.80 & 3.60 & 4.10 \\
\hline $\mathrm{Tm}$ & 0.47 & 0.59 & 0.61 & 0.64 & 0.60 & 0.58 \\
\hline $\mathrm{Yb}$ & 2.92 & 3.63 & 3.80 & 3.98 & 3.76 & 3.80 \\
\hline $\mathrm{Lu}$ & 0.46 & 0.56 & 0.59 & 0.61 & 0.58 & 0.58 \\
\hline $\mathrm{Hf}$ & 3.49 & 4.98 & 4.95 & 5.34 & 5.05 & 5.63 \\
\hline $\mathrm{Ta}$ & 0.23 & 0.18 & 0.23 & 0.32 & 0.23 & 0.29 \\
\hline $\mathrm{Pb}$ & 4.94 & 6.23 & 6.30 & 6.44 & 6.37 & 5.52 \\
\hline Th & 1.24 & 2.26 & 2.14 & 2.25 & 2.27 & 2.23 \\
\hline $\mathrm{U}$ & 0.80 & 1.38 & 1.36 & 1.43 & 1.41 & 1.30 \\
\hline Sample & TOL-13-24 & TOL-13-25 & TOL-13-38 & TOL-12-24 & TOL-12-26 & TOL-12-28 \\
\hline$\overline{\text { Complex }}$ & OT strato & OT strato & OT strato & OT strato & OT strato & OT strato \\
\hline Trend & 1 & 1 & 1 & 1 & 2 & 1 \\
\hline Lat $(\mathrm{N})$ & $55^{\circ} 51^{\prime} 44.8^{\prime \prime}$ & $55^{\circ} 51^{\prime} 43.9^{\prime \prime}$ & $55^{\circ} 51^{\prime} 48.7^{\prime \prime}$ & $55^{\circ} 49^{\prime} 17.7^{\prime \prime}$ & $55^{\circ} 49^{\prime} 16.5^{\prime \prime}$ & $55^{\circ} 49^{\prime} 16.1 "$ \\
\hline
\end{tabular}


Table 1 (continued)

\begin{tabular}{|c|c|c|c|c|c|c|}
\hline Sample & TOL-13-24 & TOL-13-25 & TOL-13-38 & TOL-12-24 & TOL-12-26 & TOL-12-28 \\
\hline Complex & OT strato & OT strato & OT strato & OT strato & OT strato & OT strato \\
\hline Long (E) & $160^{\circ} 21^{\prime} 21.8^{\prime \prime}$ & $160^{\circ} 21^{\prime} 22.4^{\prime \prime}$ & $160^{\circ} 17^{\prime} 34.9^{\prime \prime}$ & $160^{\circ} 21^{\prime} 57.8^{\prime \prime}$ & $160^{\circ} 21^{\prime} 50.2^{\prime \prime}$ & $160^{\circ} 21^{\prime} 50^{\prime \prime}$ \\
\hline $\mathrm{SiO}_{2}$ & 54.41 & 52.33 & 55.94 & 53.07 & 52.25 & 49.98 \\
\hline $\mathrm{TiO}_{2}$ & 1.05 & 1.14 & 1.05 & 1.08 & 1.47 & 1.08 \\
\hline $\mathrm{Al}_{2} \mathrm{O}_{3}$ & 17.56 & 18.39 & 18.63 & 18.31 & 18.52 & 21.58 \\
\hline $\mathrm{FeO}$ & 8.72 & 9.58 & 7.82 & 9.02 & 9.02 & 8.92 \\
\hline $\mathrm{MnO}$ & 0.18 & 0.20 & 0.16 & 0.16 & 0.14 & 0.14 \\
\hline $\mathrm{MgO}$ & 4.89 & 4.69 & 3.41 & 5.08 & 3.93 & 5.08 \\
\hline $\mathrm{CaO}$ & 8.43 & 8.87 & 7.76 & 8.62 & 8.94 & 8.77 \\
\hline $\mathrm{Na}_{2} \mathrm{O}$ & 3.18 & 3.29 & 3.60 & 3.11 & 3.50 & 3.05 \\
\hline $\mathrm{K}_{2} \mathrm{O}$ & 1.31 & 1.23 & 1.35 & 1.28 & 1.73 & 1.15 \\
\hline $\mathrm{P}_{2} \mathrm{O}_{5}$ & 0.27 & 0.28 & 0.27 & 0.27 & 0.50 & 0.26 \\
\hline $\mathrm{Cr}$ & 52 & 28 & 15 & 33 & 64 & 52 \\
\hline $\mathrm{Ni}$ & 18 & 20 & 10 & 23 & 30 & 29 \\
\hline $\mathrm{Cu}$ & 42 & 103 & 42 & 41 & 325 & 131 \\
\hline $\mathrm{Zn}$ & 98 & 106 & 98 & 83 & 112 & 77 \\
\hline $\mathrm{Rb}$ & 22.64 & 22.73 & 25.07 & 26.72 & 88.90 & 18.72 \\
\hline $\mathrm{Sr}$ & 471 & 445 & 422 & 440 & 409 & 455 \\
\hline $\mathrm{Y}$ & 19.79 & 23.69 & 23.53 & 25.10 & 50.65 & 22.96 \\
\hline $\mathrm{Zr}$ & 96 & 104 & 128 & 97 & 384 & 100 \\
\hline $\mathrm{Nb}$ & 2.74 & 2.68 & 3.78 & 2.88 & 8.45 & 2.99 \\
\hline $\mathrm{Cd}$ & 0.09 & 0.10 & 0.11 & 0.10 & 0.16 & 0.13 \\
\hline Sn & 0.61 & 0.95 & 0.51 & 0.72 & 1.59 & 0.24 \\
\hline Cs & 0.59 & 0.61 & 0.73 & 0.66 & 1.82 & 0.50 \\
\hline $\mathrm{Ba}$ & 397 & 346 & 410 & 406 & 415 & 405 \\
\hline $\mathrm{La}$ & 8.48 & 8.02 & 9.88 & 10.65 & 20.65 & 8.97 \\
\hline $\mathrm{Ce}$ & 21.11 & 20.57 & 23.55 & 25.36 & 50.90 & 21.15 \\
\hline $\mathrm{Pr}$ & 3.13 & 3.16 & 3.55 & 3.62 & 7.15 & 3.13 \\
\hline $\mathrm{Nd}$ & 15.23 & 15.83 & 17.05 & 17.53 & 33.96 & 15.32 \\
\hline $\mathrm{Sm}$ & 3.70 & 4.10 & 4.19 & 4.45 & 8.45 & 4.08 \\
\hline $\mathrm{Eu}$ & 1.22 & 1.37 & 1.38 & 1.41 & 2.32 & 1.38 \\
\hline $\mathrm{Gd}$ & 4.05 & 4.64 & 4.74 & 4.69 & 8.86 & 4.35 \\
\hline $\mathrm{Tb}$ & 0.61 & 0.71 & 0.72 & 0.70 & 1.31 & 0.68 \\
\hline Dy & 3.84 & 4.47 & 4.52 & 4.32 & 8.00 & 4.07 \\
\hline Ho & 0.78 & 0.91 & 0.93 & 0.76 & 1.40 & 0.71 \\
\hline $\mathrm{Er}$ & 2.25 & 2.64 & 2.73 & 2.43 & 4.43 & 2.23 \\
\hline $\mathrm{Tm}$ & 0.32 & 0.38 & 0.39 & 0.41 & 0.75 & 0.38 \\
\hline $\mathrm{Yb}$ & 2.14 & 2.50 & 2.66 & 2.58 & 4.58 & 2.31 \\
\hline $\mathrm{Lu}$ & 0.33 & 0.38 & 0.41 & 0.40 & 0.71 & 0.36 \\
\hline $\mathrm{Hf}$ & 2.45 & 2.61 & 3.17 & 2.60 & 6.62 & 2.65 \\
\hline $\mathrm{Ta}$ & 0.17 & 0.18 & 0.25 & 0.18 & 0.40 & 0.19 \\
\hline $\mathrm{Pb}$ & 3.22 & 2.66 & 3.78 & 3.95 & 5.82 & 3.33 \\
\hline Th & 0.84 & 0.69 & 1.12 & 1.12 & 2.42 & 0.80 \\
\hline $\mathrm{U}$ & 0.52 & 0.45 & 0.69 & 0.61 & 1.72 & 0.55 \\
\hline Sample & TOL-13-40 & TOL-12-43 & TOL-12-48 & TOL-12-50 & TOL-13-22 & TOL-13-28 \\
\hline$\overline{\text { Complex }}$ & Dike & Dike & Dike & Dike & Dike & Dike \\
\hline Trend & 1 & 1 & 2 & 2 & 1 & 1 \\
\hline Lat $(\mathrm{N})$ & $55^{\circ} 51^{\prime} 51.5^{\prime \prime}$ & $55^{\circ} 47^{\prime} 31.3^{\prime \prime}$ & $55^{\circ} 47^{\prime} 29.8^{\prime \prime}$ & $55^{\circ} 47^{\prime} 27.8^{\prime \prime}$ & $55^{\circ} 52^{\prime} 12.7^{\prime \prime}$ & $55^{\circ} 51^{\prime} 32.5^{\prime \prime}$ \\
\hline Long (E) & $160^{\circ} 23^{\prime} 13.9^{\prime \prime}$ & $160^{\circ} 23^{\prime} 35.6^{\prime \prime}$ & $160^{\circ} 24^{\prime} 23.2^{\prime \prime}$ & $160^{\circ} 24^{\prime} 21.5^{\prime \prime}$ & $160^{\circ} 20^{\prime} 36^{\prime \prime}$ & $160^{\circ} 21^{\prime} 24.4^{\prime \prime}$ \\
\hline $\mathrm{SiO}_{2}$ & 52.92 & 54.52 & 52.37 & 52.44 & 54.04 & 55.19 \\
\hline $\mathrm{TiO}_{2}$ & 1.12 & 1.03 & 1.77 & 1.70 & 1.07 & 1.00 \\
\hline $\mathrm{Al}_{2} \mathrm{O}_{3}$ & 20.26 & 18.20 & 17.87 & 18.31 & 18.00 & 19.06 \\
\hline $\mathrm{FeO}$ & 8.40 & 8.92 & 9.72 & 9.40 & 8.49 & 7.94 \\
\hline $\mathrm{MnO}$ & 0.19 & 0.15 & 0.13 & 0.13 & 0.18 & 0.16 \\
\hline $\mathrm{MgO}$ & 3.21 & 4.10 & 3.43 & 3.20 & 4.72 & 3.53 \\
\hline $\mathrm{CaO}$ & 8.83 & 8.18 & 8.32 & 8.53 & 8.18 & 7.98 \\
\hline $\mathrm{Na}_{2} \mathrm{O}$ & 3.57 & 3.34 & 3.64 & 3.62 & 3.49 & 3.45 \\
\hline $\mathrm{K}_{2} \mathrm{O}$ & 1.21 & 1.29 & 2.15 & 2.08 & 1.55 & 1.42 \\
\hline $\mathrm{P}_{2} \mathrm{O}_{5}$ & 0.29 & 0.26 & 0.60 & 0.58 & 0.29 & 0.28 \\
\hline $\mathrm{Cr}$ & 12 & 20 & 36 & 33 & 58 & 15 \\
\hline $\mathrm{Ni}$ & 11 & 12 & 20 & 21 & 30 & 10 \\
\hline $\mathrm{Cu}$ & 95 & 47 & 219 & 232 & 97 & 51 \\
\hline $\mathrm{Zn}$ & 92 & 83 & 99 & 109 & 98 & 97 \\
\hline $\mathrm{Rb}$ & 20.62 & 26.20 & 52.58 & 59.18 & 30.51 & 28.44 \\
\hline $\mathrm{Sr}$ & 469 & 434 & 400 & 473 & 454 & 458 \\
\hline $\mathrm{Y}$ & 23.84 & 24.81 & 39.65 & 40.08 & 21.05 & 21.56 \\
\hline $\mathrm{Zr}$ & 108 & 112 & 266 & 277 & 110 & 112 \\
\hline $\mathrm{Nb}$ & 2.89 & 2.80 & 6.70 & 6.70 & 3.01 & 3.55 \\
\hline $\mathrm{Cd}$ & 0.10 & 0.11 & 0.15 & 0.16 & 0.10 & 0.10 \\
\hline Sn & 0.72 & 0.67 & 0.86 & 1.09 & 0.33 & 0.64 \\
\hline Cs & 0.63 & 0.79 & 1.41 & 1.48 & 0.85 & 0.88 \\
\hline $\mathrm{Ba}$ & 356 & 460 & 596 & 605 & 426 & 388 \\
\hline $\mathrm{La}$ & 8.75 & 10.88 & 21.17 & 19.82 & 9.31 & 9.99 \\
\hline
\end{tabular}


Table 1 (continued)

\begin{tabular}{|c|c|c|c|c|c|c|}
\hline Sample & TOL-13-40 & TOL-12-43 & TOL-12-48 & TOL-12-50 & TOL-13-22 & TOL-13-28 \\
\hline Complex & Dike & Dike & Dike & Dike & Dike & Dike \\
\hline $\mathrm{Ce}$ & 22.81 & 26.03 & 50.13 & 47.87 & 23.15 & 23.22 \\
\hline $\mathrm{Pr}$ & 3.49 & 3.74 & 6.90 & 6.63 & 3.43 & 3.49 \\
\hline $\mathrm{Nd}$ & 17.36 & 18.09 & 32.22 & 31.14 & 16.54 & 16.53 \\
\hline $\mathrm{Sm}$ & 4.43 & 4.69 & 7.68 & 7.48 & 4.02 & 3.98 \\
\hline $\mathrm{Eu}$ & 1.47 & 1.50 & 2.15 & 2.14 & 1.32 & 1.30 \\
\hline Gd & 4.93 & 4.98 & 7.88 & 7.70 & 4.46 & 4.49 \\
\hline $\mathrm{Tb}$ & 0.75 & 0.74 & 1.13 & 1.10 & 0.66 & 0.66 \\
\hline Dy & 4.68 & 4.50 & 6.69 & 6.48 & 4.09 & 4.11 \\
\hline Ho & 0.96 & 0.79 & 1.16 & 1.12 & 0.83 & 0.84 \\
\hline $\mathrm{Er}$ & 2.74 & 2.48 & 3.66 & 3.53 & 2.40 & 2.44 \\
\hline $\mathrm{Tm}$ & 0.39 & 0.43 & 0.61 & 0.59 & 0.34 & 0.35 \\
\hline $\mathrm{Yb}$ & 2.62 & 2.66 & 3.76 & 3.67 & 2.27 & 2.36 \\
\hline $\mathrm{Lu}$ & 0.40 & 0.41 & 0.58 & 0.56 & 0.35 & 0.36 \\
\hline $\mathrm{Hf}$ & 2.75 & 3.03 & 5.21 & 5.07 & 2.74 & 2.77 \\
\hline $\mathrm{Ta}$ & 0.19 & 0.19 & 0.37 & 0.35 & 0.20 & 0.23 \\
\hline $\mathrm{Pb}$ & 2.82 & 4.47 & 6.30 & 6.16 & 3.76 & 3.91 \\
\hline Th & 0.81 & 1.10 & 2.07 & 1.99 & 1.00 & 1.30 \\
\hline $\mathrm{U}$ & 0.52 & 0.70 & 1.31 & 1.29 & 0.67 & 0.62 \\
\hline Sample & TOL-13-34 & TOL-13-37 & TOL-13-43 & TOL-13-75 & TOL-12-29 & TOL-13-56 \\
\hline Complex & Dike & Dike & Cone & Cone Kleshnya & Cone 1941 & Menyailov Vent \\
\hline Trend & 1 & 1 & 1 & 2 & 2 & 2 \\
\hline Lat $(\mathrm{N})$ & $55^{\circ} 51^{\prime} 50^{\prime \prime}$ & $55^{\circ} 51^{\prime} 21.8^{\prime \prime}$ & $55^{\circ} 52^{\prime} 0.7^{\prime \prime}$ & $55^{\circ} 44^{\prime} 28.1^{\prime \prime}$ & $55^{\circ} 47^{\prime} 13.2^{\prime \prime}$ & $55^{\circ} 48^{\prime} 7^{\prime \prime}$ \\
\hline Long (E) & $160^{\circ} 16^{\prime} 37.5^{\prime \prime}$ & $160^{\circ} 17^{\prime} 40.1^{\prime \prime}$ & $160^{\circ} 24^{\prime} 7^{\prime \prime}$ & $160^{\circ} 18^{\prime} 59.8^{\prime \prime}$ & $160^{\circ} 18^{\prime} 20.1^{\prime \prime}$ & $160^{\circ} 20^{\prime} 33.6^{\prime \prime}$ \\
\hline $\mathrm{SiO}_{2}$ & 52.46 & 50.10 & 51.64 & 52.00 & 50.53 & 55.01 \\
\hline $\mathrm{TiO}_{2}$ & 1.02 & 1.08 & 1.30 & 1.38 & 1.27 & 1.77 \\
\hline $\mathrm{Al}_{2} \mathrm{O}_{3}$ & 15.63 & 15.68 & 18.89 & 17.71 & 14.64 & 16.68 \\
\hline $\mathrm{FeO}$ & 9.06 & 9.85 & 9.82 & 9.29 & 9.87 & 8.92 \\
\hline $\mathrm{MnO}$ & 0.19 & 0.20 & 0.20 & 0.17 & 0.18 & 0.21 \\
\hline $\mathrm{MgO}$ & 7.74 & 7.93 & 4.51 & 4.95 & 8.89 & 3.01 \\
\hline $\mathrm{CaO}$ & 9.79 & 11.41 & 9.06 & 9.12 & 10.05 & 6.89 \\
\hline $\mathrm{Na}_{2} \mathrm{O}$ & 2.81 & 2.54 & 3.35 & 3.38 & 2.80 & 4.00 \\
\hline $\mathrm{K}_{2} \mathrm{O}$ & 1.07 & 0.97 & 0.96 & 1.56 & 1.39 & 2.71 \\
\hline $\mathrm{P}_{2} \mathrm{O}_{5}$ & 0.22 & 0.23 & 0.28 & 0.42 & 0.38 & 0.79 \\
\hline $\mathrm{Cr}$ & 199 & 181 & 22 & 56 & 745 & 17 \\
\hline $\mathrm{Ni}$ & 58 & 57 & 16 & 41 & 231 & 10 \\
\hline $\mathrm{Cu}$ & 51 & 83 & 69 & 194 & 281 & 203 \\
\hline $\mathrm{Zn}$ & 93 & 91 & 101 & 93 & 72 & 110 \\
\hline $\mathrm{Rb}$ & 17.92 & 15.78 & 14.89 & 39.38 & 41.52 & 70.28 \\
\hline $\mathrm{Sr}$ & 375 & 363 & 395 & 400 & & 348.38 \\
\hline $\mathrm{Y}$ & 18.96 & 19.33 & 26.56 & 25.83 & 28.42 & 39.43 \\
\hline $\mathrm{Zr}$ & 86 & 75 & 115 & 162 & 209 & 291 \\
\hline $\mathrm{Nb}$ & 2.30 & 1.88 & 3.14 & 5.00 & 4.93 & 6.59 \\
\hline $\mathrm{Cd}$ & 0.08 & 0.08 & 0.11 & 0.11 & 0.10 & 0.18 \\
\hline Sn & 0.59 & 1.12 & 0.21 & 0.73 & 1.48 & 1.93 \\
\hline Cs & 0.26 & 0.39 & 0.32 & 1.20 & 1.16 & 2.44 \\
\hline $\mathrm{Ba}$ & 316 & 255 & 284 & 388 & 252 & 627 \\
\hline $\mathrm{La}$ & 7.28 & 6.33 & 7.82 & 12.88 & 12.92 & 19.93 \\
\hline $\mathrm{Ce}$ & 18.51 & 16.53 & 20.95 & 30.59 & 31.66 & 53.41 \\
\hline $\operatorname{Pr}$ & 2.84 & 2.62 & 3.23 & 4.61 & 4.57 & 8.05 \\
\hline $\mathrm{Nd}$ & 14.30 & 13.58 & 16.36 & 21.81 & 22.21 & 37.32 \\
\hline $\mathrm{Sm}$ & 3.70 & 3.64 & 4.33 & 5.13 & 5.62 & 8.42 \\
\hline $\mathrm{Eu}$ & 1.21 & 1.20 & 1.44 & 1.54 & 1.54 & 2.30 \\
\hline $\mathrm{Gd}$ & 4.11 & 4.13 & 4.95 & 5.62 & 5.87 & 9.16 \\
\hline $\mathrm{Tb}$ & 0.62 & 0.64 & 0.78 & 0.82 & 0.87 & 1.32 \\
\hline Dy & 3.80 & 4.01 & 5.00 & 4.95 & 5.16 & 7.92 \\
\hline Ho & 0.76 & 0.82 & 1.03 & 1.00 & 0.89 & 1.60 \\
\hline $\mathrm{Er}$ & 2.18 & 2.32 & 2.99 & 2.87 & 2.76 & 4.60 \\
\hline $\mathrm{Tm}$ & 0.31 & 0.33 & 0.43 & 0.41 & 0.46 & 0.65 \\
\hline $\mathrm{Yb}$ & 2.02 & 2.12 & 2.87 & 2.69 & 2.77 & 4.31 \\
\hline $\mathrm{Lu}$ & 0.31 & 0.32 & 0.44 & 0.41 & 0.43 & 0.65 \\
\hline $\mathrm{Hf}$ & 2.25 & 2.04 & 2.81 & 3.66 & 4.15 & 6.36 \\
\hline $\mathrm{Ta}$ & 0.16 & 0.13 & 0.20 & 0.31 & 0.31 & 0.36 \\
\hline $\mathrm{Pb}$ & 2.05 & 1.83 & 2.11 & 3.84 & 3.45 & 7.07 \\
\hline Th & 0.75 & 0.55 & 0.61 & 1.49 & 1.36 & 2.96 \\
\hline $\mathrm{U}$ & 0.45 & 0.37 & 0.37 & 0.87 & 0.92 & 1.72 \\
\hline Sample & TOL-13-45 & TOL-13-59 & TOL-12-32 & TOL-12-33 & TOL-13-03 & TOL-13-05 \\
\hline$\overline{\text { Complex }}$ & $\overline{\text { Naboko Vent }}$ & $\overline{\text { Naboko Vent }}$ & $\overline{\text { Tolbachik pedestal }}$ & $\overline{\text { Tolbachik pedestal }}$ & $\overline{\text { Tolbachik pedestal }}$ & $\overline{\text { Tolbachik pedestal }}$ \\
\hline Trend & 2 & 2 & 1 & 1 & 1 & 1 \\
\hline Lat $(\mathrm{N})$ & $55^{\circ} 44^{\prime} 16.9^{\prime \prime}$ & $55^{\circ} 44^{\prime} 28.8^{\prime \prime}$ & $55^{\circ} 46^{\prime} 54.3^{\prime \prime}$ & $55^{\circ} 46^{\prime} 56.4^{\prime \prime}$ & $55^{\circ} 54^{\prime} 0.1^{\prime \prime}$ & $55^{\circ} 53^{\prime} 59.2^{\prime \prime}$ \\
\hline Long (E) & $160^{\circ} 5^{\prime} 30.7^{\prime \prime}$ & $160^{\circ} 18^{\prime} 14.8^{\prime \prime}$ & $160^{\circ} 14^{\prime} 13.7^{\prime \prime}$ & $160^{\circ} 13^{\prime} 45.4^{\prime \prime}$ & $160^{\circ} 22^{\prime} 44.7^{\prime \prime}$ & $160^{\circ} 21^{\prime} 31.6^{\prime \prime}$ \\
\hline $\mathrm{SiO}_{2}$ & 52.82 & 52.13 & 51.69 & 51.84 & 53.17 & 52.67 \\
\hline $\mathrm{TiO}_{2}$ & 1.99 & 2.02 & 1.08 & 1.07 & 1.25 & 0.97 \\
\hline
\end{tabular}


Table 1 (continued)

\begin{tabular}{|c|c|c|c|c|c|c|}
\hline Sample & TOL-13-45 & TOL-13-59 & TOL-12-32 & TOL-12-33 & TOL-13-03 & TOL-13-05 \\
\hline Complex & Naboko Vent & Naboko Vent & Tolbachik pedestal & Tolbachik pedestal & Tolbachik pedestal & Tolbachik pedestal \\
\hline $\mathrm{Al}_{2} \mathrm{O}_{3}$ & 16.22 & 16.33 & 14.97 & 14.89 & 18.74 & 13.80 \\
\hline $\mathrm{FeO}$ & 10.36 & 10.68 & 9.61 & 9.49 & 8.76 & 9.12 \\
\hline $\mathrm{MnO}$ & 0.20 & 0.19 & 0.16 & 0.16 & 0.18 & 0.19 \\
\hline $\mathrm{MgO}$ & 3.89 & 4.20 & 8.55 & 8.61 & 4.22 & 9.10 \\
\hline $\mathrm{CaO}$ & 7.61 & 7.58 & 10.27 & 10.26 & 8.70 & 10.64 \\
\hline $\mathrm{Na}_{2} \mathrm{O}$ & 3.69 & 3.64 & 2.65 & 2.65 & 3.49 & 2.44 \\
\hline $\mathrm{K}_{2} \mathrm{O}$ & 2.49 & 2.47 & 0.84 & 0.84 & 1.20 & 0.88 \\
\hline $\mathrm{P}_{2} \mathrm{O}_{5}$ & 0.73 & 0.75 & 0.19 & 0.18 & 0.27 & 0.20 \\
\hline $\mathrm{Cr}$ & 21 & 45 & 338 & 326 & 41 & 257 \\
\hline $\mathrm{Ni}$ & 22 & 34 & 84 & 81 & 21 & 67 \\
\hline $\mathrm{Cu}$ & 240 & 267 & 47 & 45 & 77 & 41 \\
\hline $\mathrm{Zn}$ & 100 & 118 & 74 & 73 & 105 & 84 \\
\hline $\mathrm{Rb}$ & 55.11 & 64.77 & 13.48 & 13.85 & 21.08 & 14.73 \\
\hline $\mathrm{Sr}$ & 287 & 338 & 296 & 296 & 455 & 344 \\
\hline $\mathrm{Y}$ & 32.91 & 37.72 & 21.28 & 21.36 & 23.24 & 17.40 \\
\hline $\mathrm{Zr}$ & 235 & 276 & 75 & 78 & 108 & 72 \\
\hline $\mathrm{Nb}$ & 5.44 & 6.59 & 1.86 & 2.04 & 3.20 & 1.54 \\
\hline $\mathrm{Cd}$ & 0.14 & 0.17 & 0.09 & 0.08 & 0.10 & 0.07 \\
\hline Sn & 2.03 & 0.70 & 0.83 & 0.86 & 0.68 & 0.90 \\
\hline Cs & 1.87 & 2.16 & 0.21 & 0.30 & 0.41 & 0.32 \\
\hline $\mathrm{Ba}$ & 485 & 560 & 278 & 278 & 374 & 271 \\
\hline $\mathrm{La}$ & 15.99 & 15.14 & 6.53 & 6.57 & 8.49 & 6.29 \\
\hline $\mathrm{Ce}$ & 43.20 & 49.97 & 16.16 & 16.20 & 21.83 & 16.17 \\
\hline $\operatorname{Pr}$ & 6.52 & 7.63 & 2.40 & 2.40 & 3.37 & 2.55 \\
\hline $\mathrm{Nd}$ & 30.57 & 35.64 & 12.18 & 12.15 & 16.78 & 13.10 \\
\hline $\mathrm{Sm}$ & 6.96 & 8.09 & 3.50 & 3.47 & 4.33 & 3.48 \\
\hline $\mathrm{Eu}$ & 1.88 & 2.21 & 1.15 & 1.14 & 1.45 & 1.15 \\
\hline Gd & 7.47 & 8.85 & 3.90 & 3.84 & 4.92 & 3.95 \\
\hline $\mathrm{Tb}$ & 1.08 & 1.26 & 0.61 & 0.61 & 0.75 & 0.61 \\
\hline Dy & 6.53 & 7.56 & 3.88 & 3.86 & 4.68 & 3.79 \\
\hline Ho & 1.32 & 1.52 & 0.68 & 0.68 & 0.95 & 0.77 \\
\hline $\mathrm{Er}$ & 3.76 & 4.36 & 2.13 & 2.12 & 2.70 & 2.17 \\
\hline $\mathrm{Tm}$ & 0.53 & 0.61 & 0.36 & 0.36 & 0.38 & 0.31 \\
\hline $\mathrm{Yb}$ & 3.51 & 4.07 & 2.18 & 2.18 & 2.53 & 2.01 \\
\hline $\mathrm{Lu}$ & 0.53 & 0.61 & 0.33 & 0.33 & 0.38 & 0.31 \\
\hline $\mathrm{Hf}$ & 5.04 & 6.03 & 2.16 & 2.20 & 2.90 & 2.07 \\
\hline $\mathrm{Ta}$ & 0.28 & 0.33 & 0.09 & 0.13 & 0.22 & 0.07 \\
\hline $\mathrm{Pb}$ & 5.57 & 6.28 & 2.39 & 2.83 & 3.10 & 1.83 \\
\hline Th & 2.34 & 2.61 & 0.65 & 0.66 & 0.79 & 0.64 \\
\hline $\mathrm{U}$ & 1.35 & 1.54 & 0.39 & 0.40 & 0.54 & 0.42 \\
\hline Sample & TOL-13-07 & TOL-13-11 & TOL-13-14 & POV-10-01 & POV-10-02 & POV-10-03 \\
\hline Complex & Tolbachik pedestal & Tolbachik pedestal & Tolbachik pedestal & Mount Povorotnaya & Mount Povorotnaya & Mount Povorotnaya \\
\hline Trend & 1 & 1 & 1 & 1 & 1 & 1 \\
\hline Lat $(\mathrm{N})$ & $55^{\circ} 53^{\prime} 58.9^{\prime \prime}$ & $55^{\circ} 53^{\prime} 45.6^{\prime \prime}$ & $55^{\circ} 53^{\prime} 3.5^{\prime \prime}$ & $55^{\circ} 53^{\prime} 7.6^{\prime \prime}$ & $55^{\circ} 53^{\prime} 3.6^{\prime \prime}$ & $55^{\circ} 53^{\prime} 38.4^{\prime \prime}$ \\
\hline Long (E) & $160^{\circ} 21^{\prime} 38.6^{\prime \prime}$ & $160^{\circ} 21^{\prime} 56.6^{\prime \prime}$ & $160^{\circ} 20^{\prime} 6.1^{\prime \prime}$ & $160^{\circ} 27^{\prime} 43.5^{\prime \prime}$ & $160^{\circ} 27^{\prime} 21.4^{\prime \prime}$ & $160^{\circ} 27^{\prime} 5.7^{\prime \prime}$ \\
\hline $\mathrm{SiO}_{2}$ & 52.26 & 53.89 & 56.73 & 56.16 & 54.21 & 53.24 \\
\hline $\mathrm{TiO}_{2}$ & 0.98 & 0.97 & 0.87 & 0.98 & 0.96 & 0.96 \\
\hline $\mathrm{Al}_{2} \mathrm{O}_{3}$ & 13.88 & 14.30 & 18.93 & 18.23 & 16.15 & 14.56 \\
\hline $\mathrm{FeO}$ & 9.19 & 8.83 & 7.18 & 7.40 & 8.25 & 8.46 \\
\hline $\mathrm{MnO}$ & 0.19 & 0.18 & 0.15 & 0.14 & 0.15 & 0.15 \\
\hline $\mathrm{MgO}$ & 9.22 & 8.14 & 2.98 & 3.76 & 6.63 & 8.85 \\
\hline $\mathrm{CaO}$ & 10.78 & 9.90 & 7.67 & 7.28 & 8.94 & 9.38 \\
\hline $\mathrm{Na}_{2} \mathrm{O}$ & 2.42 & 2.60 & 3.74 & 3.94 & 3.15 & 2.89 \\
\hline $\mathrm{K}_{2} \mathrm{O}$ & 0.88 & 0.99 & 1.44 & 1.79 & 1.33 & 1.25 \\
\hline $\mathrm{P}_{2} \mathrm{O}_{5}$ & 0.20 & 0.20 & 0.30 & 0.32 & 0.23 & 0.26 \\
\hline $\mathrm{Cr}$ & 271 & 254 & 16 & 11 & 147 & 384 \\
\hline $\mathrm{Ni}$ & 68 & 64 & 12 & 10 & 32 & 85 \\
\hline $\mathrm{Cu}$ & 50 & 49 & 54 & 196 & 41 & 66 \\
\hline $\mathrm{Zn}$ & 85 & 86 & 98 & 76 & 69 & 70 \\
\hline $\mathrm{Rb}$ & 14.99 & 15.47 & 28.67 & 32.67 & 20.80 & 16.17 \\
\hline $\mathrm{Sr}$ & 345 & 363 & 478 & 419 & 420 & 468 \\
\hline $\mathrm{Y}$ & 17.71 & 17.36 & 22.77 & 24.80 & 19.56 & 19.40 \\
\hline $\mathrm{Zr}$ & 74 & 76 & 132 & 123 & 92 & 89 \\
\hline $\mathrm{Nb}$ & 1.49 & 1.85 & 3.89 & 3.33 & 2.52 & 1.87 \\
\hline $\mathrm{Cd}$ & 0.08 & 0.08 & 0.11 & 0.10 & 0.09 & 0.08 \\
\hline Sn & 1.52 & 0.89 & 0.33 & 0.79 & 0.89 & 1.01 \\
\hline Cs & 0.38 & 0.34 & 0.61 & 0.94 & 0.57 & 0.43 \\
\hline $\mathrm{Ba}$ & 272 & 283 & 439 & 753 & 570 & 587 \\
\hline $\mathrm{La}$ & 6.37 & 6.51 & 11.53 & 11.99 & 10.12 & 10.83 \\
\hline $\mathrm{Ce}$ & 16.36 & 16.52 & 27.38 & 28.28 & 23.40 & 25.38 \\
\hline $\operatorname{Pr}$ & 2.60 & 2.59 & 4.04 & 4.00 & 3.30 & 3.62 \\
\hline Nd & 13.36 & 13.23 & 18.78 & 19.26 & 15.77 & 17.32 \\
\hline
\end{tabular}


Table 1 (continued)

\begin{tabular}{|c|c|c|c|c|c|c|}
\hline \multirow{2}{*}{$\frac{\text { Sample }}{\text { Complex }}$} & \multirow{2}{*}{$\frac{\text { TOL-13-07 }}{\text { Tolbachik pedestal }}$} & \multirow{2}{*}{$\frac{\text { TOL-13-11 }}{\text { Tolbachik pedestal }}$} & \multirow{2}{*}{$\frac{\text { TOL-13-14 }}{\text { Tolbachik pedestal }}$} & POV-10-01 & POV-10-02 & POV-10-03 \\
\hline & & & & Mount Povorotnaya & Mount Povorotnaya & Mount Povorotnaya \\
\hline $\mathrm{Sm}$ & 3.57 & 3.47 & 4.34 & 4.91 & 3.98 & 4.20 \\
\hline $\mathrm{Eu}$ & 1.17 & 1.14 & 1.40 & 1.55 & 1.27 & 1.30 \\
\hline Gd & 4.05 & 3.93 & 4.79 & 5.14 & 4.10 & 4.23 \\
\hline $\mathrm{Tb}$ & 0.62 & 0.60 & 0.71 & 0.75 & 0.59 & 0.58 \\
\hline Dy & 3.89 & 3.71 & 4.34 & 4.49 & 3.54 & 3.32 \\
\hline Ho & 0.79 & 0.75 & 0.89 & 0.79 & 0.62 & 0.57 \\
\hline $\mathrm{Er}$ & 2.22 & 2.12 & 2.61 & 2.47 & 1.95 & 1.77 \\
\hline $\mathrm{Tm}$ & 0.31 & 0.30 & 0.38 & 0.41 & 0.32 & 0.29 \\
\hline $\mathrm{Yb}$ & 2.06 & 1.96 & 2.59 & 2.55 & 1.99 & 1.78 \\
\hline Lu & 0.31 & 0.30 & 0.40 & 0.40 & 0.31 & 0.27 \\
\hline $\mathrm{Hf}$ & 2.17 & 2.12 & 3.29 & 3.35 & 2.54 & 2.29 \\
\hline Ta & 0.06 & 0.09 & 0.26 & 0.22 & 0.16 & 0.12 \\
\hline $\mathrm{Pb}$ & 2.34 & 2.10 & 3.90 & 5.86 & 4.19 & 3.75 \\
\hline Th & 0.66 & 0.65 & 1.26 & 1.53 & 1.10 & 1.05 \\
\hline $\mathrm{U}$ & 0.43 & 0.42 & 0.76 & 0.94 & 0.78 & 0.67 \\
\hline Sample & POV-10-04 & POV-10-06 & POV-10-07 & PLAT-13-03 & PLAT-13-04 & PLAT-13-06 \\
\hline Complex & Mount Povorotnaya & Mount Povorotnaya & Mount Povorotnaya & KGV basement & KGV basement & KGV basement \\
\hline Trend & 1 & 1 & 1 & & & \\
\hline Lat (N) & $55^{\circ} 53^{\prime} 17.3^{\prime \prime}$ & $55^{\circ} 53^{\prime} 45.3^{\prime \prime}$ & $55^{\circ} 53^{\prime} 53.1^{\prime \prime}$ & $55^{\circ} 55^{\prime} 14.2^{\prime \prime}$ & $55^{\circ} 55^{\prime} 12^{\prime \prime}$ & $55^{\circ} 55^{\prime} 10.3^{\prime \prime}$ \\
\hline Long (E) & $160^{\circ} 27^{\prime} 18^{\prime \prime}$ & $160^{\circ} 27^{\prime} 42.4^{\prime \prime}$ & $160^{\circ} 28^{\prime} 58^{\prime \prime}$ & $160^{\circ} 18^{\prime} 4.6^{\prime \prime}$ & $160^{\circ} 18^{\prime} 5.1^{\prime \prime}$ & $160^{\circ} 18^{\prime} 6^{\prime \prime}$ \\
\hline $\mathrm{SiO}_{2}$ & 54.37 & 54.18 & 53.96 & 55.06 & 55.45 & 55.11 \\
\hline $\mathrm{TiO}_{2}$ & 0.97 & 0.99 & 1.24 & 1.72 & 1.86 & 1.75 \\
\hline $\mathrm{Al}_{2} \mathrm{O}_{3}$ & 16.13 & 17.10 & 17.92 & 16.77 & 16.46 & 16.89 \\
\hline $\mathrm{FeO}$ & 8.26 & 8.16 & 9.07 & 9.32 & 9.20 & 8.92 \\
\hline $\mathrm{MnO}$ & 0.14 & 0.15 & 0.16 & 0.17 & 0.16 & 0.15 \\
\hline $\mathrm{MgO}$ & 6.55 & 5.63 & 3.76 & 3.36 & 3.20 & 3.46 \\
\hline $\mathrm{CaO}$ & 8.78 & 9.03 & 8.31 & 7.09 & 6.92 & 7.21 \\
\hline $\mathrm{Na}_{2} \mathrm{O}$ & 3.16 & 3.30 & 3.49 & 3.74 & 3.71 & 3.63 \\
\hline $\mathrm{K}_{2} \mathrm{O}$ & 1.39 & 1.24 & 1.76 & 2.17 & 2.34 & 2.22 \\
\hline $\mathrm{P}_{2} \mathrm{O}_{5}$ & 0.25 & 0.23 & 0.33 & 0.61 & 0.70 & 0.65 \\
\hline $\mathrm{Cr}$ & 145 & 91 & 39 & 16 & 14 & 20 \\
\hline $\mathrm{Ni}$ & 35 & 27 & 17 & 27 & 26 & 26 \\
\hline $\mathrm{Cu}$ & 52 & 70 & 61 & 265 & 239 & 201 \\
\hline $\mathrm{Zn}$ & 75 & 69 & 70 & 103 & 103 & 96 \\
\hline $\mathrm{Rb}$ & 23.41 & 16.11 & 31.16 & 49.99 & 54.85 & 51.76 \\
\hline $\mathrm{Sr}$ & 462 & 401 & 444 & 384 & 354 & 369 \\
\hline $\mathrm{Y}$ & 20.86 & 19.89 & 21.78 & 34.18 & 37.02 & 34.38 \\
\hline $\mathrm{Zr}$ & 99 & 97 & 135 & 264 & 282 & 259 \\
\hline $\mathrm{Nb}$ & 2.70 & 2.62 & 3.59 & 6.13 & 5.52 & 4.91 \\
\hline $\mathrm{Cd}$ & 0.09 & 0.08 & 0.10 & 0.16 & 0.19 & 0.17 \\
\hline Sn & 0.72 & 1.04 & 0.60 & 1.96 & 2.45 & 2.18 \\
\hline Cs & 0.60 & 0.26 & 0.78 & 1.20 & 2.09 & 1.88 \\
\hline $\mathrm{Ba}$ & 596 & 417 & 627 & 687 & 713 & 685 \\
\hline $\mathrm{La}$ & 10.58 & 8.85 & 12.72 & 16.04 & 22.70 & 18.80 \\
\hline $\mathrm{Ce}$ & 24.54 & 20.72 & 29.21 & 46.81 & 52.16 & 48.89 \\
\hline $\mathrm{Pr}$ & 3.46 & 2.94 & 4.00 & 7.08 & 7.89 & 7.30 \\
\hline $\mathrm{Nd}$ & 16.49 & 14.13 & 18.43 & 32.99 & 36.75 & 34.04 \\
\hline $\mathrm{Sm}$ & 4.11 & 3.64 & 4.39 & 7.52 & 8.34 & 7.72 \\
\hline $\mathrm{Eu}$ & 1.30 & 1.17 & 1.36 & 1.99 & 2.15 & 2.03 \\
\hline Gd & 4.25 & 3.84 & 4.50 & 8.07 & 9.09 & 8.43 \\
\hline $\mathrm{Tb}$ & 0.61 & 0.57 & 0.64 & 1.16 & 1.29 & 1.20 \\
\hline Dy & 3.60 & 3.49 & 3.81 & 6.97 & 7.76 & 7.17 \\
\hline Ho & 0.63 & 0.61 & 0.67 & 1.39 & 1.54 & 1.43 \\
\hline $\mathrm{Er}$ & 1.97 & 1.91 & 2.13 & 3.95 & 4.38 & 4.08 \\
\hline $\mathrm{Tm}$ & 0.33 & 0.32 & 0.36 & 0.55 & 0.61 & 0.57 \\
\hline $\mathrm{Yb}$ & 2.03 & 2.01 & 2.26 & 3.62 & 4.04 & 3.75 \\
\hline $\mathrm{Lu}$ & 0.31 & 0.30 & 0.35 & 0.55 & 0.61 & 0.57 \\
\hline $\mathrm{Hf}$ & 2.61 & 2.58 & 3.44 & 6.21 & 6.73 & 6.14 \\
\hline $\mathrm{Ta}$ & 0.17 & 0.17 & 0.22 & 0.37 & 0.31 & 0.28 \\
\hline $\mathrm{Pb}$ & 4.41 & 4.03 & 5.49 & 7.92 & 9.00 & 8.31 \\
\hline Th & 1.13 & 1.02 & 1.46 & 2.72 & 3.19 & 2.93 \\
\hline $\mathrm{U}$ & 0.81 & 0.63 & 0.91 & 1.63 & 1.95 & 1.80 \\
\hline
\end{tabular}

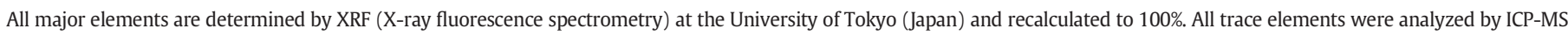

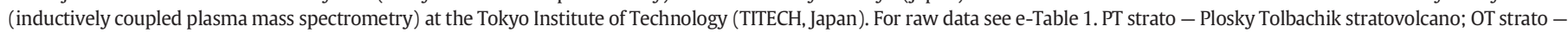
Ostry Tolbachik stratovolcano. FeO is the total Fe content expressed as FeO. Major elements are presented in wt.\%, trace elements - in ppm. 
Table 2

Results of the $\mathrm{K}-\mathrm{Ar}$ isotope dating of the volcanic rocks from the Tolbachik pedestal and Mount Povorotnaya.

\begin{tabular}{|c|c|c|c|c|c|}
\hline Sample & Geological object & $\begin{array}{l}\mathrm{K}_{2} \mathrm{O} \\
((\mathrm{wt} . \%)\end{array}$ & $\begin{array}{l}{ }^{40} \mathrm{Ar}_{\mathrm{rad}} \\
\left(10^{-6} \mathrm{ml} \mathrm{STP} / \mathrm{g}\right)\end{array}$ & $\begin{array}{l}\text { Age }( \pm 1 \mathrm{~s}) \\
(\mathrm{Ma})\end{array}$ & $\begin{array}{l}{ }^{40} \mathrm{Ar}_{\text {air }} \\
(\%)\end{array}$ \\
\hline TOL-13-03 & Tolbachik basement, northern part, augite-phyric & 1.320 & 0.0037 & $0.086 \pm 0.016$ & 97 \\
\hline POV-10-05 & Mount Povorotnaya, lowest flow, augite-phyric & 1.450 & 0.0143 & $0.306 \pm 0.010$ & 84.1 \\
\hline
\end{tabular}

performed using a stainless steel ultra-high vacuum extraction line. Samples of $0.35-0.4 \mathrm{~g}$ were wrapped in 10- $\mu \mathrm{m}$ copper foil and fused at $1500{ }^{\circ} \mathrm{C}$ using a double-vacuum tantalum resistance furnace following a bakeout at $120{ }^{\circ} \mathrm{C}$ for $72 \mathrm{~h}$. An ${ }^{38} \mathrm{Ar}$ spike was removed from the tank using a pipette valve and was mixed with the sample gas during fusion of the sample. Purification of the sample gas was achieved with two SAES Getters NP-10 Sorb-Ac pumps and one zirconium and titanium plate getter. Argon isotopes were measured on a VG Isotopes $1200 \mathrm{C}$ mass spectrometer equipped with a Nier-type ion source and a single Faraday collector. Argon isotopes of air standard were analyzed once or twice a day for mass discrimination correction.

The K content was determined based on about $200 \mathrm{mg}$ of each sample by flame emission spectrometry, using peak integration and a lithium internal standard method (Matsumoto, 1989). Error for K analysis was estimated to be $0.5 \%$ based on replicate analyses of reference materials (Matsumoto, 1989).

\section{Results and discussion}

\subsection{Petrography}

Tolbachik massif rocks can be divided into three associations and 8 types based on petrography. They are Ol-bearing rocks $(\mathrm{Ol}-\mathrm{Cpx}-\mathrm{Pl}$, $\mathrm{Ol}-\mathrm{Pl}, \mathrm{Ol}-\mathrm{Cpx}$, and $\mathrm{Ol}-\mathrm{Opx}-\mathrm{Pl}$ ), Ol-free lavas (2Px-Pl, $\mathrm{Cpx}-\mathrm{Pl}$, and $\mathrm{Pl}$ ), and subaphyric rocks. About $80 \%$ of all Tolbachik rocks are Ol-bearing. The samples from Mount Povorotnaya are similar to those of the pedestal rocks and belong to the $\mathrm{Cpx}-\mathrm{Pl}$ association, including the presence of augite-phyric rocks. All rock types show a range of vesicularities (from dense to highly vesicular) and phenocryst abundances.

Olivine-clinopyroxene-plagioclase lavas are the most common for the Tolbachik massif. They are represented at all stages of volcano activity: they occur in the pedestal rocks, compose the main part of Ostry and Plosky stratovolcanoes, the majority of cinder cone-lava fields, and are found as megaplagiophyric rocks in dikes. These rocks are characterized by porphyritic and serioporphyritic textures as well as their megaplagiophyric varieties in dikes. The modes of mafic minerals vary from $5 \%$ to $20 \%$. The relative proportions of phenocrysts, the proportions of $\mathrm{Ol}, \mathrm{Cpx}$ and $\mathrm{Pl}$, as well as rock porosity varies significantly from subaphyric to megaporphyritic, from Ol-Cpx-rich to substantially Plrich, and from dense to highly porous lavas. Groundmass textures are hyalopilitic, pilotaxitic, or microdoleritic. The groundmass comprises the same minerals, with the color changing from light gray to black depending on magnetite ( $\mathrm{mt}$ ) abundance. The groundmass can change inside one sample from vitrophyric to holocrystalline. All phenocrysts are euhedral. Plagioclase is often the most abundant phenocryst in Ol-Cpx$\mathrm{Pl}$ rocks, forming twins and intergrowths. The Pl size varies from several millimeters to $2 \mathrm{~cm}$ in megaplagiophyric lavas. Plagioclase phenocrysts are often strongly resorbed in the core or along mantle zones. Clinopyroxene also has many intergrowth forms and sometimes has inclusions of Ol. Olivine normally is less than $3 \mathrm{~mm}$ in size and locally contains melt inclusions and spinel (Sp).

Olivine-plagioclase rocks are represented in all parts of the volcanic complex except for the pedestal suite. They are the prevalent rocks at Ostry Tolbachik stratovolcano. The textures of these rocks are most commonly subaphyric and they are characterized by a lack of Cpx crystals, or contain sub-phenocrysts less than $1 \mathrm{~mm}$ in size. In amount and size, Pl phenocrysts are dominant. Olivine is normally less than $2 \mathrm{~mm}$ in size, but reaches $1 \mathrm{~cm}$ in megaplagiophyric lavas. Groundmass textures typically vary from pilotaxitic or vitrophyric to cryptofelsitic in cinder-like varieties, and are rarely hyalopilitic and intersertal. Olivine-Pl rocks are characterized by the presence of quenched volcanic glass.

Olivine-clinopyroxene lavas are less common compared to the first two types. These associations exist at the pedestal, stratovolcano and dike complexes. Among the monogenetic cones such associations were observed only in high-Mg basalts of the 1941 and 1975-1976 eruptions. The textures of these rocks vary from subaphyric to highly porphyritic. The modes of mafic minerals vary from $1 \%$ to $15 \%$ and the sizes of minerals reach up to $1 \mathrm{~cm}$. Olivine is unaltered, euhedral, frequently has $\mathrm{Sp}$ inclusions, and is intergrown with Cpx. Groundmass textures are vitrophyric and hyalopilitic. Volcanic glass appears to be brown due to a high content of Mt microlites.

Olivine-orthopyroxene-plagioclase rocks are rare in the massif and were observed only in the upper part of Ostry Tolbachik. The amount of Pl is ten times higher than that of the mafic minerals. The texture of these samples is hyalopilitic with elongated opx phenocrysts.

Clinopyroxene-plagioclase lavas are only prevalent among the pedestal rocks and were found in the lower parts of both stratovolcanoes and in the dike complexes. The amount of $\mathrm{Cpx}$ and $\mathrm{Pl}$ may vary dramatically from Pl-rich rocks (see below) to Cpx-rich samples (e.g. augite-phyric rocks) without Pl phenocrysts. The rocks have a porphyritic texture with up to $20 \%$ Cpx as the only phenocryst. Olivine grains less than $1 \mathrm{~mm}$ in size are observed very rarely. Clinopyroxene crystals and/or intergrowths up to $8-10 \mathrm{~mm}$ frequently have zoning and can be surrounded by glass. In Cpx-Pl lavas Pl is elongated, its crystals do not exceed 2-3 mm, they often have zoning and resorption. The groundmass typically has hyalopilitic, vitrophyric, or microdoleritic textures. Clinopyroxene-plagioclase and especially augite-phyric rocks are abundant within the pedestal complex and are very typical for Mount Povorotnaya.

Two-pyroxene-plagioclase rocks are rarely observed; similar to CpxPl lavas they exist in the pedestal suite, in stratovolcanoes, and dikes. Despite higher content of Pl in these rocks, Opx crystals can be up to $1 \mathrm{~cm}$ in size, zoned and their mode can reach up to $20 \%$.

Pl-rich samples occur in all complexes of the massif, but they prevail in the upper parts of stratovolcanoes and in monogenetic cinder cone/ lava field, including the 2012-2013 eruption. Olivine and Cpx can also occur in these rocks, but their mode does not exceed $1 \%$ of the less than 1-3 mm size. Pl crystals and intergrowth of euhedral shape have different sizes forming serioporphyritic structure of the rocks. The ground mass structure is hyalopilitic or vitrophyric.

Subaphyric rocks were also found in all parts of the complex, but as opposed to Pl-rich samples, they rarely occur in the monogenetic cinder cone/lava fields. They are characterized by fine textures, different degrees of groundmass crystallization, and the lack of any phenocrysts. Olivine, Cpx, Opx and Pl microlites occur in the groundmass. The fresh glass color varies from clear to brown. The groundmass texture is hyalopilitic or vitrophyric.

\subsection{Major and trace elements}

\subsubsection{Major elements}

All studied rocks of Tolbachik volcanic massif belong to a medium-K and high-K tholeiitic basalt-basaltic-andesitic series (Fig. 4a, b; Table 1, e-Table 1). The detailed data, however, show 
some systematic differences in the trends for all major element diagrams. On Harker diagrams (Fig. 4), the Tolbachik suite splits into two trends, as well as a separate area, which includes only samples from the first three days of the 2012 eruption (the so-called Menyailov Vent). However, on plots of major elements vs. MgO samples from the Menyailov Vent clearly belong to one of the mentioned trends. The first trend (trend 1 ) consists of medium-K basalts and basaltic andesites (Figs. 4, 5, trend 1). This trend takes a boundary position between the tholeiitic and calc-alkaline fields on a Miyashiro diagram with a slight slope to tholeiitic field from basalts to basaltic andesites (Fig. $4 \mathrm{~b}$ ). In the basalt to basaltic andesite range this trend shows increasing $\mathrm{K}_{2} \mathrm{O}, \mathrm{Na}_{2} \mathrm{O}$, and $\mathrm{P}_{2} \mathrm{O}_{5}$, but decreasing $\mathrm{Al}_{2} \mathrm{O}_{3}, \mathrm{TiO}_{2}, \mathrm{CaO}, \mathrm{FeO}_{\mathrm{T}}$, and $\mathrm{MgO}$. The major elements in lavas of Mount Povorotnaya are plotted similar to those of trend 1.

The second trend (trend 2) is medium-high-K and is in line with most of previously published data for Tolbachik. With increasing $\mathrm{SiO}_{2}$ content, even by only $2.5 \mathrm{wt}$.\%, the trend shows a sharp increase in alkalis, $\mathrm{P}_{2} \mathrm{O}_{5}, \mathrm{TiO}_{2}$ and a sharp decrease in $\mathrm{MgO}, \mathrm{MnO}, \mathrm{FeO}_{\mathrm{T}}$, and $\mathrm{CaO}$ (Figs. 4, 5 , trend 2). The major elements in KGV basement lavas are similar to those of the Menyailov Vent samples. Trend 2 is enriched in $\mathrm{K}_{2} \mathrm{O}, \mathrm{TiO}_{2}$, $\mathrm{FeO}_{\mathrm{T}}, \mathrm{P}_{2} \mathrm{O}_{5}$ and depleted in $\mathrm{SiO}_{2}$ and $\mathrm{Al}_{2} \mathrm{O}_{3}$ compared to trend 1 (Figs. 4, 5).

\subsubsection{Trace elements}

Trace element patterns for all studied rocks have typical arc signatures with strong but variable LILE and LREE enrichment and low HFSE, which are consistent with fluid addition to all studied rocks (Fig. 6). Despite rather uniform trace element curves for all studied samples, the two groups of rocks have some systematic differences. Trend 1 samples show consistently lower values for REE, $\mathrm{Nb}$, and Ta compared to the samples of trend 2 . The rocks from the Menyailov Vent are mostly enriched in all incompatible elements not only within the Tolbachik massif lavas, but also within all rocks of the KGV. High-Mg basalts of the 1975-1976 Northern Breakthrough analyzed in this study and from previously published data (e.g. Churikova et al., 2001) show the lowest concentrations in all incompatible elements at Tolbachik massif. They clearly demonstrate trace element distributions similar to the distribution in samples from trend 1. The high-K rocks of the 1975-1976 Southern Breakthrough and 1941 eruptions have similar trace element distributions as in trend 2 . The samples from trend 1 are systematically depleted in Nb, Ta, and REE compared to NMORB, while the rocks within trend 2 are systematically enriched. The trace elements in lavas of Mount Povorotnaya plot similarly to those of trend 1. On trace element and trace element ratio binary diagrams, the samples of two trends are also consistently different (Fig. 7a, b).

\subsubsection{Isotope data}

Isotope data for the Tolbachik volcanic massif and surrounding volcanic vents plot close to the NMORB field and within the previously reported data ranges for CKD (Figs. 8 and 9; Table 3). However, unlike the previously published data for the massif (Kersting and Arculus, 1995; Tatsumi et al., 1995; Kepezhinskas et al., 1997; Turner et al., 1998; Volynets et al., 2000; Churikova et al., 2001; Dosseto et al., 2003; Bindeman et al., 2004; Portnyagin et al., 2007a), some of our data show isotope ratios enriched in Sr- and depleted in Nd- (Fig. 9) with the exception of one sample from Plosky Tolbachik (Dosseto et al., 2003).

Due to a more representative sampling, we can identify some systematic changes within the different units of the Tolbachik massif. There is a general increase in ${ }^{87} \mathrm{Sr} /{ }^{86} \mathrm{Sr}$ and a less pronounced decrease in ${ }^{143} \mathrm{Nd} /{ }^{144} \mathrm{Nd}$ across the entire range of data. Samples of basalt from cinder cones and upper lava flows of Plosky Tolbachik summit are low in $\mathrm{Sr}$ - and high in Nd-isotope ratios and are plotted the closest to NMORB values. The rocks of the pedestal, stratovolcanoes, and dikes are higher in ${ }^{87} \mathrm{Sr} /{ }^{86} \mathrm{Sr}$ at nearly the same or slightly lower ${ }^{143} \mathrm{Nd} /{ }^{144} \mathrm{Nd}$ ratios. Mount Povorotnaya samples have the highest $\mathrm{Sr}$ - and the lowest
Nd-isotope ratios (Fig. 8). The KGV plateau lavas are practically identical in $\mathrm{Sr}-\mathrm{Nd}$-isotope space with pedestal/stratovolcano/dike complexes.
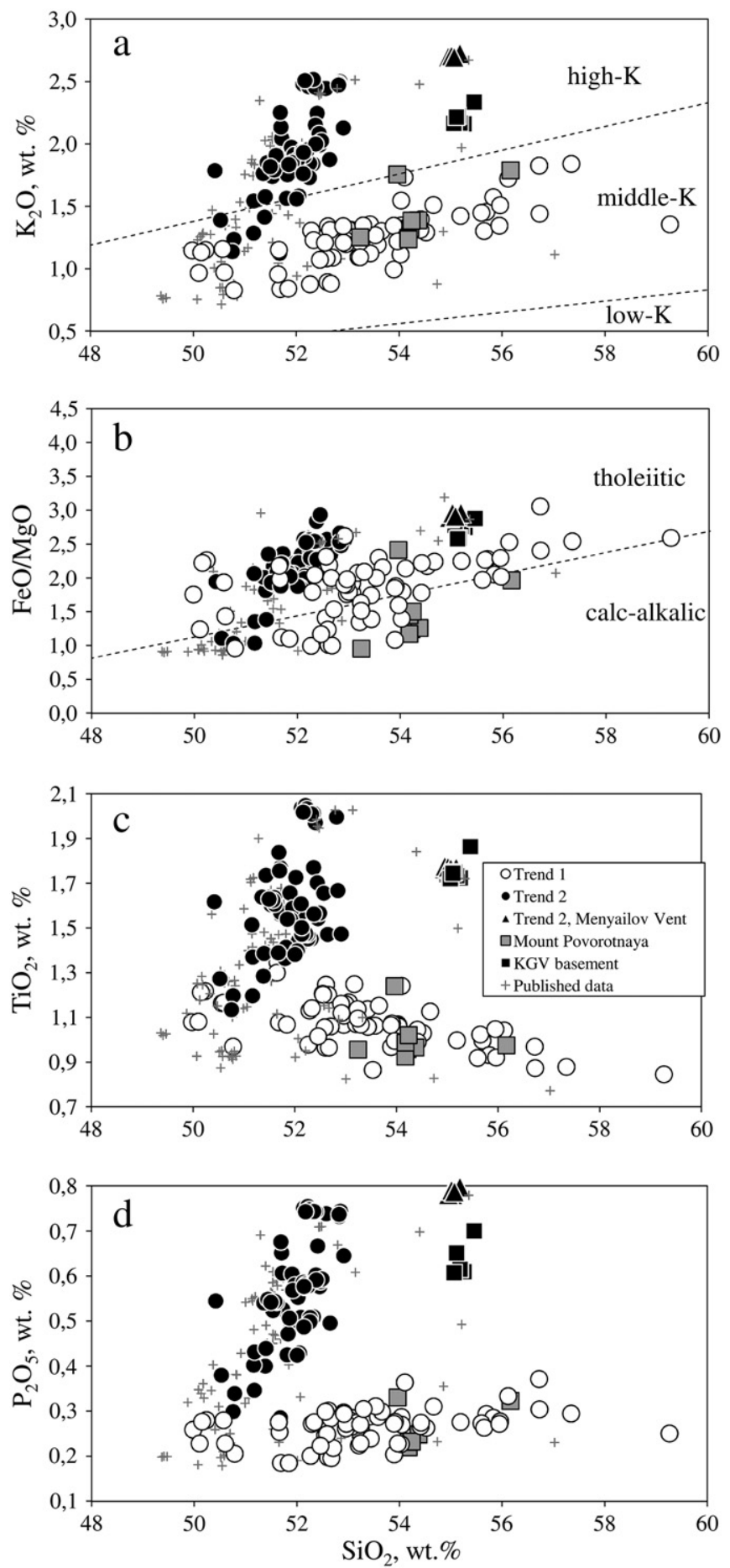

Fig. 4. Harker diagrams for Tolbachik volcanic massif rocks, Mount Povorotnaya and KGV basement. (a) $\mathrm{K}_{2} \mathrm{O}$ vs. $\mathrm{SiO}_{2}$, classification lines after Le Maitre et al. (2002) showing fields of low-, medium-, and high-K arc lavas. (b) $\mathrm{FeO} / \mathrm{MgO}$ vs. $\mathrm{SiO}_{2}$, classification lines after Miyashiro (1974) showing fields of tholeiitic and calc-alkaline rocks. (c-d) $\mathrm{TiO}_{2}$ and $\mathrm{P}_{2} \mathrm{O}_{5}$ vs. $\mathrm{SiO}_{2}$, showing the splitting of all Tolbachik rocks into two trends. Menyailov Vent and KGV basement rocks are enriched in $\mathrm{SiO}_{2}$ compared to lavas of trend 2. Previously published data shown for comparison.

Data are from several sources (Kersting, 1995; Tatsumi et al., 1995; Hochstaedter et al., 1996; Kepezhinskas et al., 1997; Turner et al., 1998; Pineau et al., 1999; Churikova et al., 2001; Bindeman et al., 2004; Portnyagin et al., 2007a; Bryant et al., 2011; Volynets et al., 2013). 

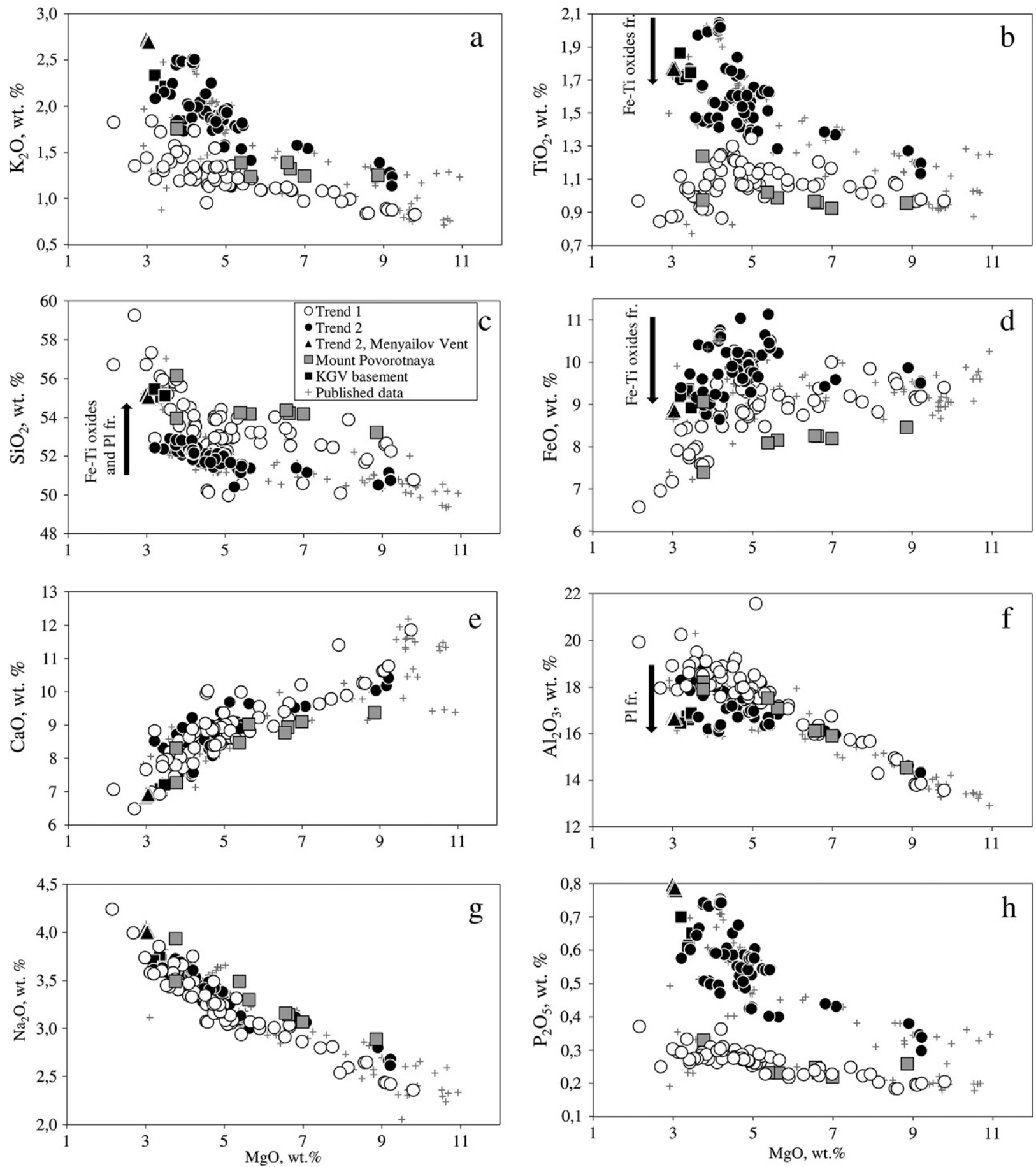

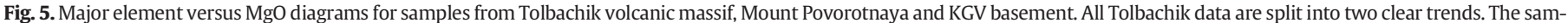

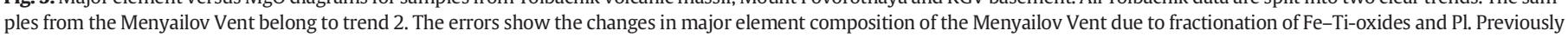
published data shown for comparison. The data sources are the same as in Fig. 4.

\subsubsection{K-Ar dating}

To clarify the geochronology of the Tolbachik volcanic massif, we determined the age of two oldest rocks in this area by a K-Ar method, analyzing one sample from the pedestal complex of the volcano and one sample from Mount Povorotnaya. Both samples are augite-phyric basalts with large (up to $2 \mathrm{~cm}$ ) Cpx phenocrysts, unaltered and sampled from the lowest lava flow in each suite. The age of pedestal rocks is $0.086 \pm 0.016 \mathrm{Ma}$, and is considered to be the beginning of the Tolbachik volcanic massif formation. The sample of Mount Povorotnaya gives an age of $0.306 \pm 0.01 \mathrm{Ma}$ (Fig. 3a, Table 2).

\section{Interpretation}

\subsection{Geochemical evolution of the Tolbachik volcanic massif}

Samples from the pedestal complex form trend 1 (Fig. 10a, b) and show large variations in major elements: $51-56$ wt.\% $\mathrm{SiO}_{2}, 13-19$ wt.\% $\mathrm{Al}_{2} \mathrm{O}_{3}, 3-9$ wt.\% $\mathrm{MgO}$ at rather similar alkalis and $\mathrm{P}_{2} \mathrm{O}_{5}$ values (2.43.7 wt.\% $\mathrm{Na}_{2} \mathrm{O}, 0.9-1.4$ wt.\% $\mathrm{K}_{2} \mathrm{O}, 0.2-0.3$ wt.\% $\mathrm{P}_{2} \mathrm{O}_{5}$ ) and $\mathrm{Mg} \# 43-64$. Trace element distribution paths in all pedestal rocks are very similar and subparallel, and have some enrichment within the basalt to basaltic 


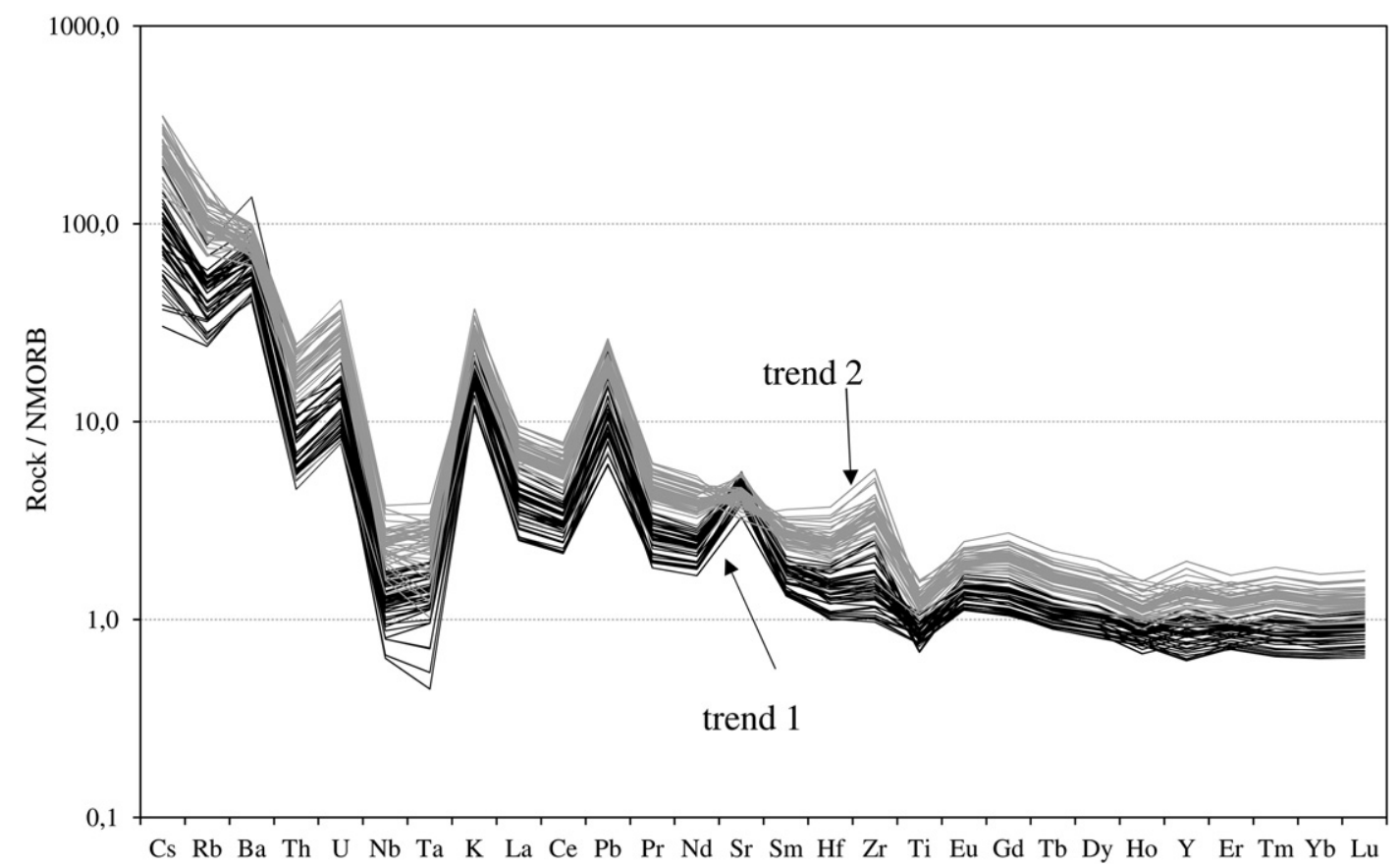

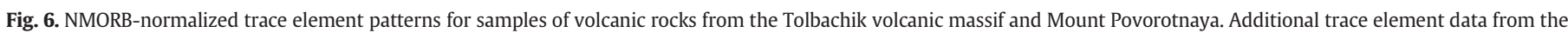

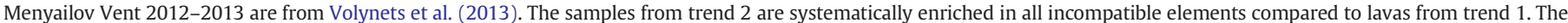
order of incompatible elements is derived from Hofmann (1988) with Cs and all REE added. NMORB values after Sun and McDonough (1989).

andesite range, providing clear evidence of fractional crystallization (Fig. 11a). The rocks of this complex are the most depleted ones compared to all other rocks of the massif with the depletion factor (derived from the relative HREE concentrations of N-MORB and studied rocks), relative to N-MORB source, of about 1/1.6.

Samples of Ostry Tolbachik stratovolcano are distributed across both trends (Fig. 10c-d). All samples from the lower part of stratovolcano, up to an elevation of $2000 \mathrm{~m}$, and several samples from the upper part fall into trend 1 , while the majority of samples from the upper part belong to trend 2. Trace element distributions for samples of trend 1 and 2 are also systematically different (Fig. 11b). Trace element patterns from trend 1 samples have a well-expressed positive $\mathrm{Sr}$ anomaly with the $\mathrm{Rb}_{\mathrm{n}} / \mathrm{Ba}_{\mathrm{n}}<1$ ("n" throughout this paper refers to normalized values of trace elements to N-MORB), whereas those from trend 2 show a very weak positive $\mathrm{Sr}$ anomaly and $\mathrm{Rb}_{\mathrm{n}} / \mathrm{Ba}_{\mathrm{n}}>1$. Because the values of the partition coefficients between silicate melt and plagioclase decrease in the following order $\mathrm{Sr}>\mathrm{Ba}>\mathrm{Rb}$, the observed difference between two trends in the Sr anomaly and in the $\mathrm{Rb}_{\mathrm{n}} / \mathrm{Ba}_{\mathrm{n}}$ ratio could indicate more extensive plagioclase crystallization for trend 2 than for trend 1 . Furthermore, trace element concentrations within trend 2 are two times higher in almost all incompatible elements compared to those within trend 1 (Fig. 11b). While the depletion factor for samples within trend 1 varies from $1 / 1.15$ to $1 / 1.4$, in trend 2 rocks the factor ranges from $1 / 0.7$ to $1 / 0.8$

Plosky Tolbachik stratovolcano is also composed of rocks falling within both trends (Figs. 10e-f and 11c). Similar to samples from Ostry Tolbachik, the lavas from the low part of Plosky Tolbachik fall within trend 1 , and in the upper part of the stratovolcano lavas falling within both trends are observed; but in Plosky Tolbachik this sequence starts from an elevation of $1400 \mathrm{~m}$. All Plosky Tolbachik stratovolcano trace element patterns, falling within trends 1 and 2, are similar to the ones for Ostry Tolbachik samples.

The samples of the dike complex also fall within both trends on both volcanoes overlapping with stratovolcano lavas in major and trace elements and their ratios (Figs. 10g-h and 11d). The similarity of dikes and stratovolcanoes in compositional range suggests that the dikes are the feeders to stratovolcano lavas and that they have close genetic relationships and were formed from the same parental melts under comparable P-T conditions.

The major and trace element compositions of lavas from most of the monogenetic cones (including the historical 1941 eruption, high-K basalts of the 1975-1976, and lavas of 2012-2013 eruption) are part of trend 2 (Figs. 10i-j and 11e). These lavas are characterized by weak positive or negligible negative $\mathrm{Sr}$ anomalies with $\mathrm{Rb}_{\mathrm{n}} / \mathrm{Ba}_{\mathrm{n}}>1$ or close to 1 . The lavas of 2012-2013 are the most evolved in the trend, and no rocks with similar major element compositions have been previously found at the massif. Nevertheless, in terms of trace element compositions similar lavas were erupted at older cones in southwest-west sector of the Tolbachik massif (samples TOL-12-30 and TOL-12-31).

The samples of the Menyailov Vent differ from other rocks of trend 2 on some major element diagrams (Figs. 4, 5). The main differences are in higher $\mathrm{SiO}_{2}$ and lower $\mathrm{Al}_{2} \mathrm{O}_{3}, \mathrm{FeO}$ and $\mathrm{TiO}_{2}$ contents (shown as arrows in Fig. 5). These differences can be explained by simultaneous crystallization of Fe-Ti-oxides and plagioclase, which is consistent with macroscopic observations. The change in $\mathrm{TiO}_{2}$ content in trend 1 indicates that Fe-Ti-oxide saturation occurred in magmas containing about $4.5 \mathrm{wt} . \%$ $\mathrm{MgO}$, while in trend 2 the crystallization of Fe-Ti-oxide started at 3.5\% MgO. This difference can also be observed in a plot of $\mathrm{Cr} / \mathrm{Ni}$ vs. $\mathrm{TiO}_{2}$ (Fig. 7e). Fractionation of Fe-Ti-oxide in trend 1 started at a $\mathrm{Cr} / \mathrm{Ni}$ ratio in the melt of approximately 2 , while in trend 2 it started only at 0.5 .

The high-Mg basalts of the 1975-1976 Northern Breakthrough, lava of the monogenetic cone to the northeast of Plosky Tolbachik (TOL-1343), and sample TOL-96-04b (Churikova et al., 2001) are the most mafic samples from the monogenetic cones. Their compositions plot at a crossover position and can be attributed in terms of major elements to either of the two trends. However, in terms of trace element compositions these lavas belong to trend 1 (Fig. 11e). The rocks of these monogenetic cones are most depleted in HREE and are characterized by a more pronounced negative $\mathrm{Nb}-\mathrm{Ta}$ anomaly compared to samples from trend 2 . The trace element patterns of these lavas are very similar to those of pedestal rocks with positive $\mathrm{Sr}$ anomalies and $\mathrm{Rb}_{\mathrm{n}} / \mathrm{Ba}_{\mathrm{n}}<1$. 

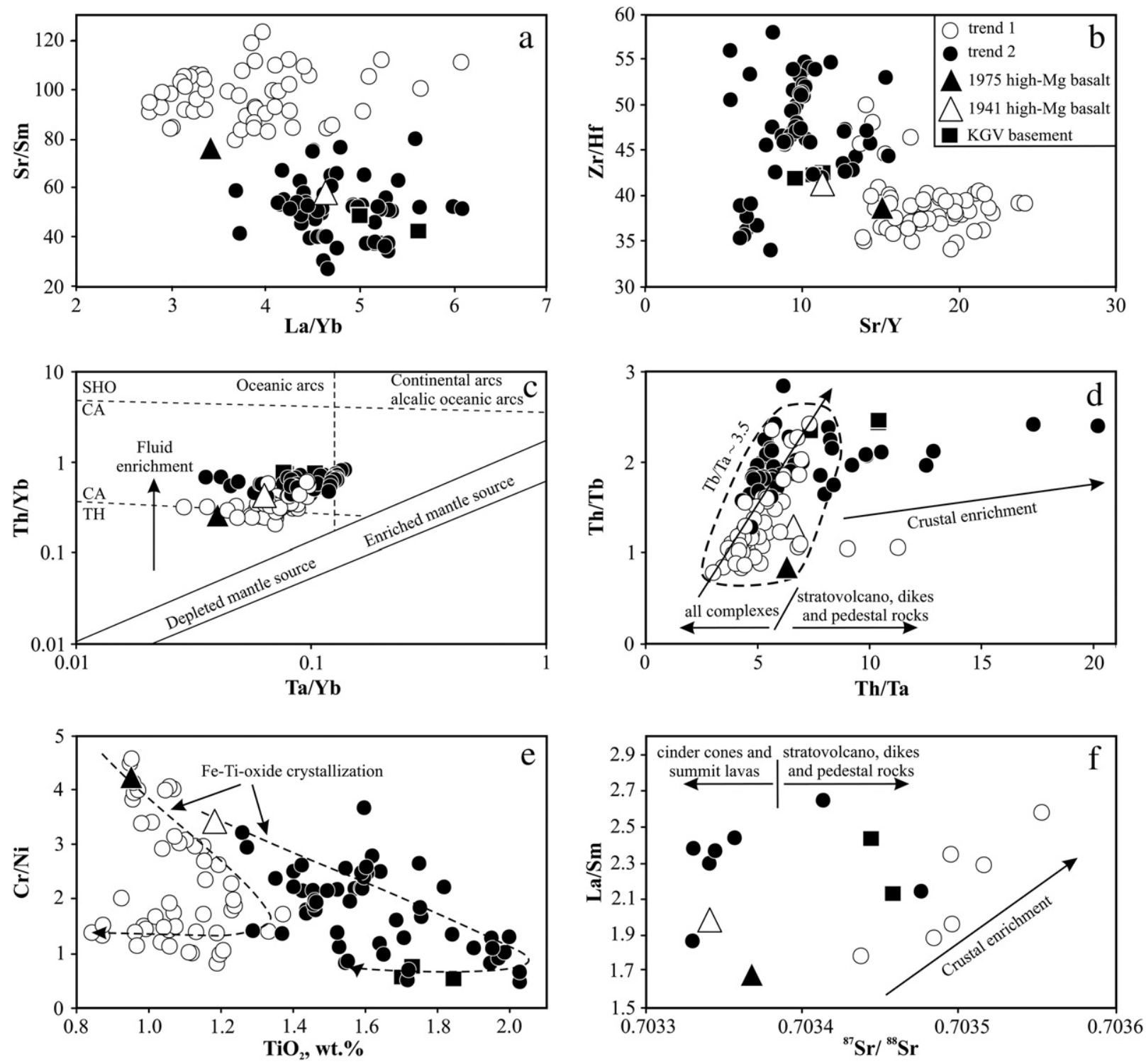

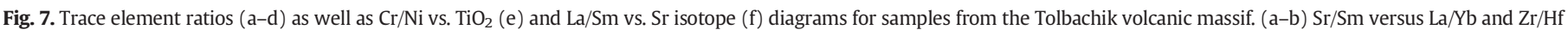

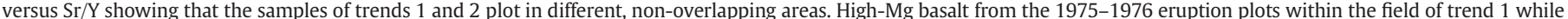

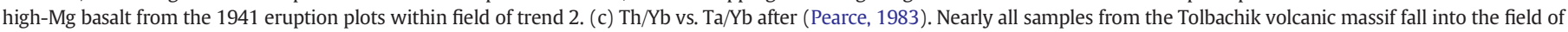

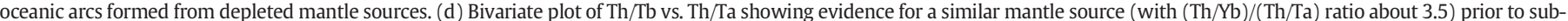

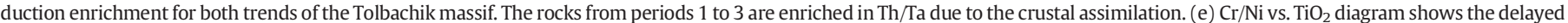

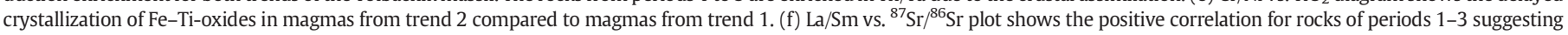
small amounts of crustal assimilation. Holocene cinder cone lavas have variable La/Sm ratio at relatively narrow range in Sr-isotopes. Symbols as in Fig. 7.

Thus, trend 1 covers all samples of the massif pedestal, the lower parts of stratovolcanoes up to elevations of 1400-2000 masl, most of the upper parts of stratovolcanoes, some dikes, high-Mg basalts of 1975-1976 Northern Breakthrough and sample TOL-13-43. Trend 1 samples are depleted in HREE relative to N-MORB and have an $\mathrm{Rb}_{\mathrm{n}} /$ $\mathrm{Ba}_{\mathrm{n}}<1$, and some of them are depleted in HFSE. Trend 1 is dominated by fractional crystallization processes and is typical of many arc magma series and for the KGV area (e.g. Churikova et al., 2001, 2013).

Trend 2 comprises the following samples: the upper parts of stratovolcanoes, some dikes and the majority of the monogenetic cinder cone and lava fields, including samples from the Southern Breakthrough of 1975-1976, the 2012-2013 eruptions and most of the previously published data. The samples from trend 2 are all enriched in LILE, HFSE and REE relative to N-MORB and have $\mathrm{Rb}_{\mathrm{n}} / \mathrm{Ba}_{\mathrm{n}}<1$. Trend 2 has an unusually steep slope on the $\mathrm{SiO}_{2}-\mathrm{K}_{2} \mathrm{O}$ diagram (Fig. 4a), shows a clear tholeiitic signature (Fig. 4b) and is not typical for the KGV. Previously, the origin of this trend was explained by having two mantle sources, high-Mg for the 1975-1976 Northern Breakthrough and high-K for Southern Breakthrough of 1975-1976, that mixed (Flerov et al., 1984), or by the addition of different amounts of slab-derived fluids (Churikova et al., 2001). Low Sr isotopic values for all Tolbachik monogenetic cones (Figs. 8, 9) rule out crustal contamination processes during formation of trend 2 lavas.

\subsection{Fractional crystallization}

Systematic differences in distributions of samples from trend 1 and trend 2 in major (Figs. 4, 5 and 10) and trace (Figs. 6 and 11) elements suggest that these two trends cannot be related by the same processes from the same source magma. We hypothesized 


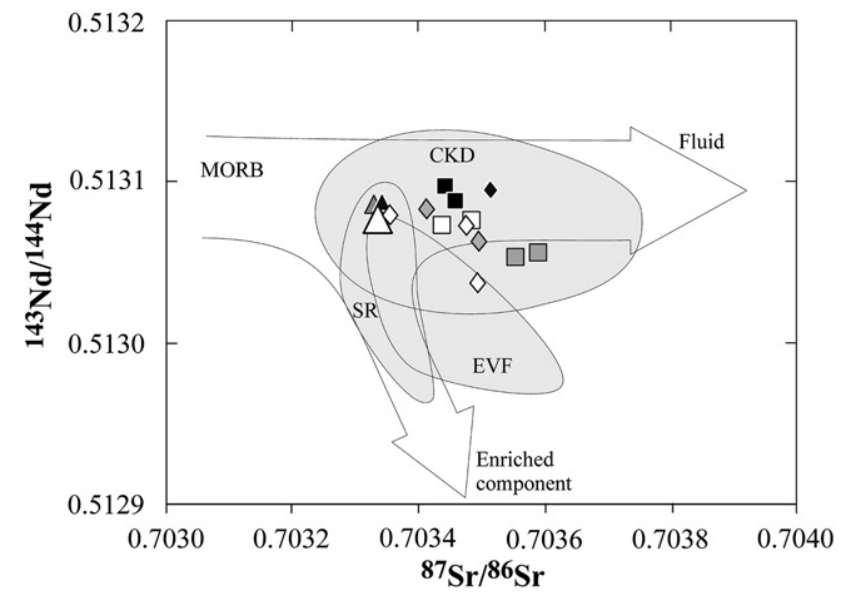

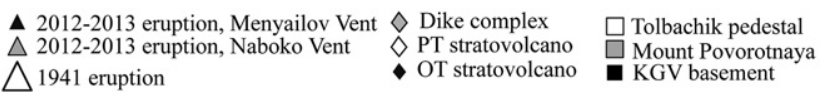

Fig. 8. ${ }^{143} \mathrm{Nd} /{ }^{144} \mathrm{Nd} \mathrm{vs}{ }^{87} \mathrm{Sr} /{ }^{86} \mathrm{Sr}$ for the different rock complexes of the Tolbachik volcanic massif, Mount Povorotnaya and KGV basement. EVF - Eastern Volcanic Front, CKD Central Kamchatka Depression, SR - Sredinny Range, PT - Plosky Tolbachik, OT - Ostry Tolbachik.

The data for CKD field are from several sources (Kersting, 1995; Turner et al., 1998, 2007; Churikova et al., 2001; Dosseto et al., 2003; Bindeman et al., 2004, 2005; Portnyagin et al., 2007a; Almeev et al., 2013), the arrows and data for EVF and SR are from Churikova et al. (2001).

that the main process of rock formation within trend 1 and trend 2 is fractional crystallization at different conditions. In order to constrain the crystallization conditions we used published results based on mineral compositions as well as experimental studies (Flerov et al., 1980; Lukanin et al., 1980; Romanchev and Flerov, 1980; Flerov et al., 2015; see also Table 3 in Churikova et al., 2015). Then we used the COMAGMAT 3.57 software (Ariskin, 1999) to calculate the fractional crystallization paths for trend 1 and trend 2 .

\subsubsection{Parental melts}

Two high-Mg lavas, one of the 1975-1976 Northern Breakthrough (sample 655) and the other of the 1941 eruption (sample 201) that have been previously analyzed for major and trace elements (Churikova et al., 2001; Fig. 12), are used as parental melts. Despite

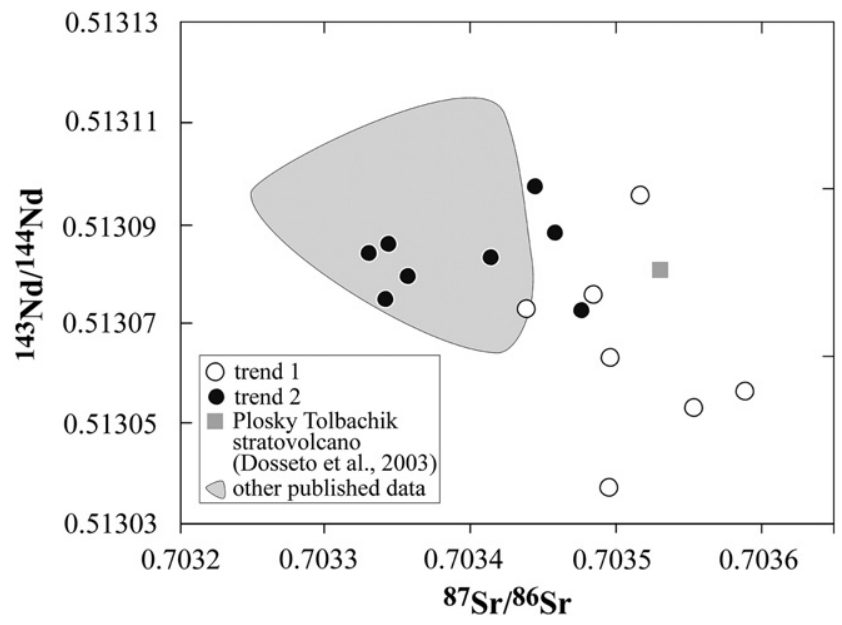

Fig. 9. ${ }^{143} \mathrm{Nd} /{ }^{144} \mathrm{Nd} \mathrm{vs}{ }^{87} \mathrm{Sr} /{ }^{86} \mathrm{Sr}$ for samples from trend 1 (white circles) and trend 2 (black circles). Gray field - previously published data on the Tolbachik monogenetic lavas.

Data are from several published sources (Kersting and Arculus, 1995; Tatsumi et al., 1995; Kepezhinskas et al., 1997; Churikova et al., 2001; Dosseto et al., 2003; Bindeman et al., 2004; Portnyagin et al., 2007a).
Table 3

Sr- and Nd-isotope compositions of selected rocks of the Tolbachik volcanic massif, Mount Povorotnaya and KGV basement.

\begin{tabular}{|c|c|c|c|c|c|c|}
\hline Sample & $\begin{array}{l}\text { Sample } \\
\text { description }\end{array}$ & Trend & ${ }^{87} \mathrm{Sr} /{ }^{86} \mathrm{Sr}$ & $\begin{array}{l} \pm 2 \\
\text { s.e. }\end{array}$ & ${ }^{143} \mathrm{Nd} /{ }^{144} \mathrm{Nd}$ & $\begin{array}{l} \pm 2 \\
\text { s.e. }\end{array}$ \\
\hline \multicolumn{7}{|c|}{ Tolbachik pedestal } \\
\hline TOL-13-07 & Northern sector & 1 & 0.703438 & \pm 6 & 0.513073 & \pm 8 \\
\hline TOL-12-33 & SW sector & 1 & 0.703484 & \pm 7 & 0.513076 & \pm 10 \\
\hline \multicolumn{7}{|c|}{ Plosky Tolbachik stratovolcano } \\
\hline TOL-12-15 & Crater cone & 2 & 0.703357 & \pm 6 & 0.513080 & \pm 9 \\
\hline TOL-12-38 & SE sector & 2 & 0.703476 & \pm 7 & 0.513073 & \pm 9 \\
\hline TOL-12-53 & Eastern sector & 1 & 0.703495 & \pm 7 & 0.513037 & \pm 9 \\
\hline \multicolumn{7}{|c|}{ Ostry Tolbachik stratovolcano } \\
\hline TOL-13-24 & Northern sector & 1 & 0.703516 & \pm 8 & 0.513096 & \pm 8 \\
\hline \multicolumn{7}{|l|}{ Dike complex } \\
\hline TOL-12-50 & Plosky Tolbachik dike & 2 & 0.703414 & \pm 6 & 0.513083 & \pm 8 \\
\hline TOL-13-34 & Ostry Tolbachik dike & 1 & 0.703496 & \pm 7 & 0.513063 & \pm 8 \\
\hline \multicolumn{7}{|c|}{ Monogenetic cinder-lava cones } \\
\hline TOL-12-29 & 1941 eruption & 2 & 0.703341 & \pm 6 & 0.513075 & \pm 4 \\
\hline TOL-13-56 & Menyailov Vent & 2 & 0.703344 & \pm 6 & 0.513086 & \pm 7 \\
\hline TOL-13-59 & Naboko Vent & 2 & 0.703330 & \pm 6 & 0.513084 & \pm 6 \\
\hline \multicolumn{7}{|c|}{ Povorotnaya Mount } \\
\hline POV-10-03 & Low part & 1 & 0.703553 & \pm 7 & 0.513053 & \pm 7 \\
\hline POV-10-04 & Upper part & 1 & 0.703589 & \pm 7 & 0.513056 & \pm 7 \\
\hline \multicolumn{7}{|c|}{ KGV basement } \\
\hline PLAT-13-03 & Upper part & & 0.703458 & \pm 7 & 0.513088 & \pm 7 \\
\hline PLAT-13-06 & Low part & & 0.703444 & \pm 6 & 0.513097 & \pm 9 \\
\hline
\end{tabular}

very similar major element compositions and high Mg\# (65.5 for high-Mg 1975-1976 Northern Breakthrough and 64 for 1941 eruption) both samples show different trace element distributions. Trace elements in high-Mg basalts of 1975-1976 Northern Breakthrough are similar to trace elements in lavas within trend 1 with $\mathrm{Rb}_{\mathrm{n}} / \mathrm{Ba}_{\mathrm{n}}<1$ and clear positive $\mathrm{Sr}$ anomaly. At the same time, trace element distribution in 1941 eruption basalts is identical to trace element distributions in lavas within trend 2 with $\mathrm{Rb}_{\mathrm{n}} / \mathrm{Ba}_{\mathrm{n}}>1$ and a weak positive $\mathrm{Sr}$ anomaly. In addition to that, in 1941 basalts all incompatible elements, $\mathrm{K}_{2} \mathrm{O}$, $\mathrm{P}_{2} \mathrm{O}_{5}$, and $\mathrm{TiO}_{2}$, are twice as high relative to high-Mg basalts of 19751976 Northern Breakthrough.

Due to higher partition coefficients of $\mathrm{Sr}$ and $\mathrm{Ba}$ compared to $\mathrm{Rb}$ in plagioclase (Bindeman and Davis, 2000), it is possible that the $\mathrm{Rb} / \mathrm{Ba}$ ratio and $\mathrm{Sr}$ anomaly could have been affected by Pl crystallization and fractionation. However, neither high-Mg lavas of 1975-1976 nor 1941 lavas have Pl phenocrysts so change in these ratios cannot be explained by Pl crystallization. Also it is impossible to get a two-fold enrichment occurring in all incompatible elements between two high-Mg basalts just due to fractional crystallization.

This difference in trace element compositions could be explained by one of the following: different melting conditions of the same mantle source, two different mantle sources, or the same but variably depleted mantle source.

Churikova et al. (2001) showed that the degree of melting for middle-K and high-K rocks from the massif is rather similar and that high-K lavas are enriched in fluid mobile elements compared to middle-K ones, which is confirmed by the $\mathrm{Th} / \mathrm{Yb}$ enrichment of lavas from trend 2 compared to rocks of trend 1 (Fig. 7c). Higher fluid addition would result in lower melting temperature in the source region and a higher degree of melting for samples from trend 2 with subsequent depletion in trace element abundances compared to trend 1. Meanwhile, in trend 2 samples we observe just the opposite correlation. Therefore, different melting conditions of the same mantle source are unlikely.

Parallel trace element patterns and very similar isotopic values in both samples (Figs. 6, 8 and 9) suggest that they could have originated from the same source. Using the approach of Saunders et al. (1988) we examined the variability of three highly incompatible elements 

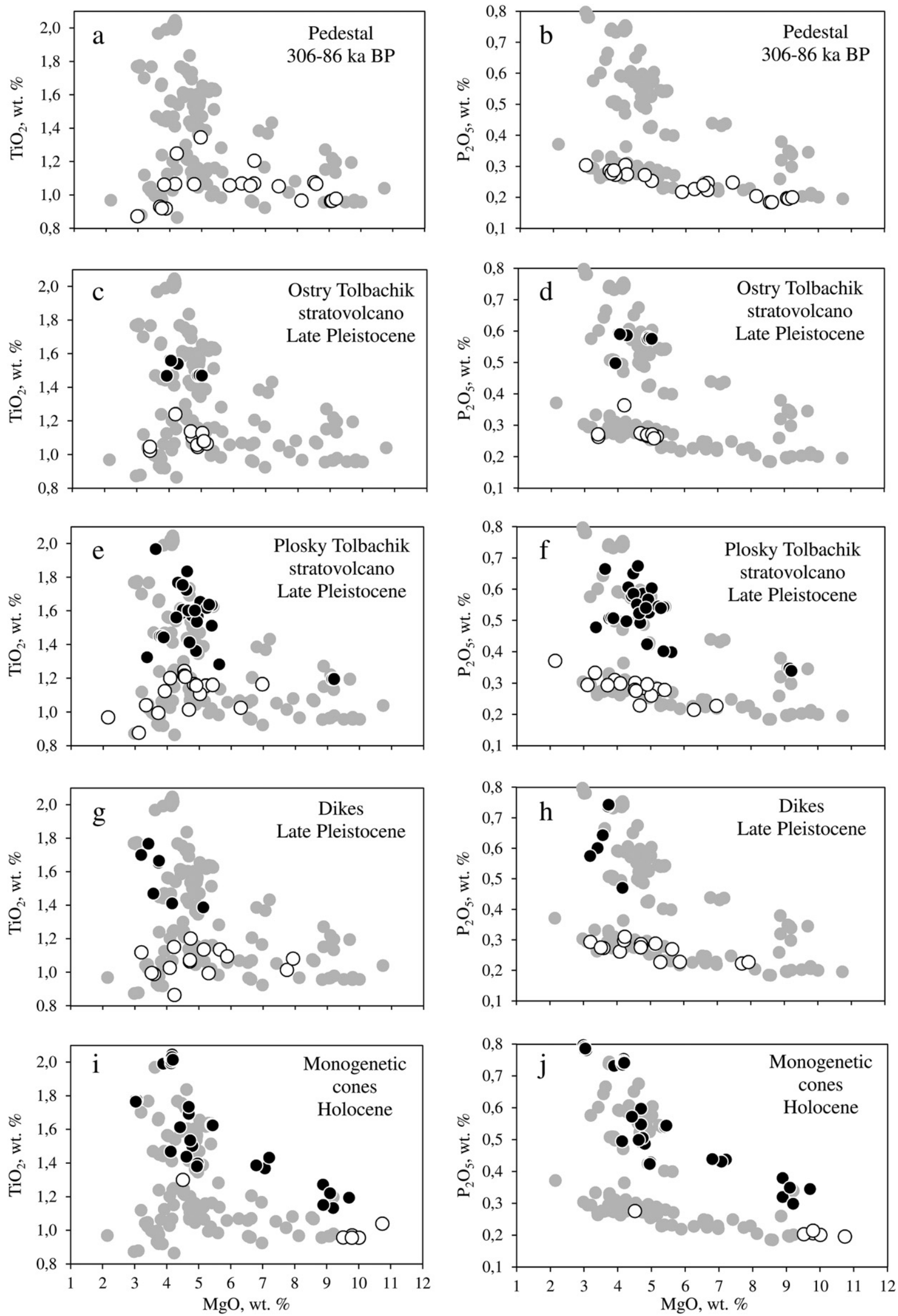

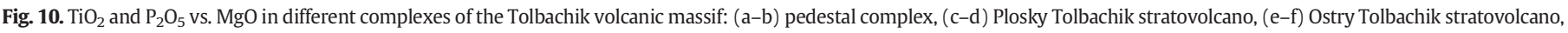
$(\mathrm{g}-\mathrm{h})$ dike complex, and $(\mathrm{i}-\mathrm{j})$ monogenetic cones. The ages for volcanic complexes are written on each panel. The gray points show all studied samples. See text for discussion. 

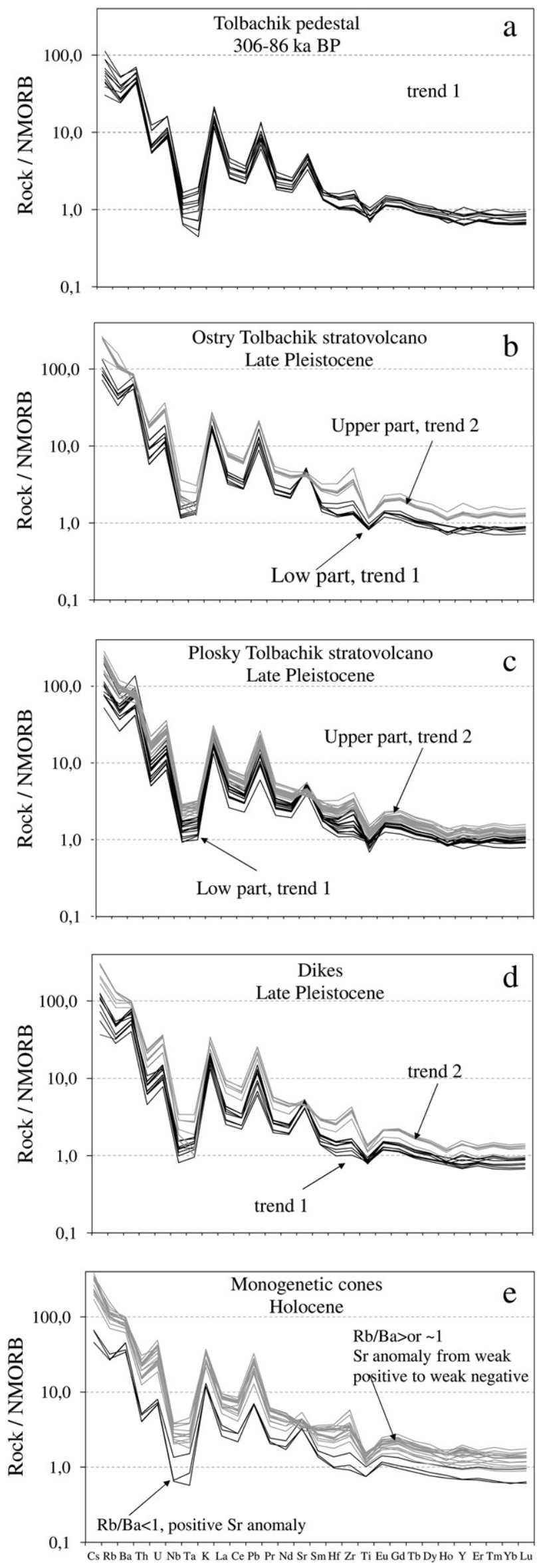

that have identical bulk partitioning coefficients (Th, Tb and Ta) for all samples of the Tolbachik volcanic massif (Fig. 7d). All cinder cone lavas and most rocks from other complexes show nearly the same ( Th/Ta)/(Th/Tb) ratio of approximately 3.5 , suggesting the same mantle source prior to subduction enrichment for all Tolbachik massif rocks. The Th-enrichment, due to the subduction slab fluid (or melt), does not change the $(\mathrm{Th} / \mathrm{Ta}) /(\mathrm{Th} / \mathrm{Tb})$ ratio of these rocks. Some enrichment in $\mathrm{Th} / \mathrm{Ta}$ ratio is observed for samples from the stratovolcanoes, dikes, and basement, which could suggest some crustal assimilation during periods 1-3 (Fig. 7d). On Th/Yb vs. Ta/Yb diagram all samples from the Tolbachik massif also fall in the same field within the depleted mantle sources (Fig. 7c). Some samples of trend 2 take the intermediate position between volcanic and continental arc. All of these observations are consistent with the rocks of the Tolbachik massif having originated from the same, but variably depleted mantle source.

On many trace element discrimination diagrams, samples from trend 1 fall into the field of volcanic arcs, while the lavas from trend 2 show a rift-like signature (e.g. Fig. 13). We suggest that during basement and stratovolcano formation the melts originated from the depleted mantle source supplying lavas of trend 1 to the surface (e.g. sample 655). After KGV extension in the Late Pleistocene-Holocene and formation of the rift-like structure at the Tolbachik massif, the lower, less depleted and more fluid enriched layers of the same mantle source were upwelling and produced the more enriched parental melts of trend 2 (e.g. sample 201).

On trace element ratio diagrams, high-Mg samples of the 1975-1976 Northern Breakthrough fall close to the trend 1 field while samples of 1941 lava are within trend 2 (Fig. 7a, b). Based on the differences in trace element distributions, we suggest that the high-Mg melt of the 1975-1976 Northern Breakthrough is the parental melt for trend 1 and the lava of 1941 is the parental melt for trend 2. But, because the high-Mg samples of 1975-1976 Northern Breakthrough are plotted at the crossover of two trends on major element diagrams, this melt potentially can be the parental melt for both trends at different P-T$\mathrm{H}_{2} \mathrm{O}-\mathrm{fO}_{2}$ conditions. Thus, sample 655 (1975-1976 Northern Breakthrough) was selected as a parental melt for modeling of trend 1, and two samples were selected as the parental melts for modeling of trend 2: 655 (1975-1976 Northern Breakthrough) and 201 (1941 eruption).

\subsubsection{Simulation approach}

Trend 1 comprises samples from the pedestal, stratovolcanoes, dikes, one cone on the northern slope of Plosky Tolbachik (sample TOL-13-43) and initial high-Mg lavas of 1975-1976 Northern Breakthrough. The pressure of crystallization estimated for stratovolcano rocks by $\mathrm{Ol}-\mathrm{Cpx}$-melt equilibrium is $2-3 \mathrm{kbar}$ at $2-3 \mathrm{wt} . \% \mathrm{H}_{2} \mathrm{O}$ (Flerov et al., 2015). A fractional crystallization model was tested in a series of computations at $1-5 \%$ of $\mathrm{H}_{2} \mathrm{O}$, NNO oxygen buffer, and pressures of $1-$ 10 kbar under constant pressure, assuming that the magma chamber was not at a significantly different depth during melt evolution, and at polybaric conditions. For sample 655 with water content of $3 \%$ and a constant pressure of $2 \mathrm{kbar}$, the modeled compositions fall close to the observed magma compositions of trend 1 at Tolbachik massif (Fig. 14).

Trend 2 comprises mainly samples from the stratovolcanoes, dikes, and cinder cone-lava field samples. Experiments on rocks of the 19751976 eruption suggested that high-Mg basalts of 1975-1976 Northern Breakthrough were formed under anhydrous conditions at initial crystallization pressures of 4-7 kbar and final crystallization pressures of

Fig. 11. Distribution of trace elements in different complexes of the Tolbachik volcanic massif: (a) pedestal complex, (b) Plosky Tolbachik stratovolcano, (c) Ostry Tolbachik stratovolcano, (d) dike complex, and (e) monogenetic cones. Even though all trace element patterns are subparallel (except $\mathrm{Rb}, \mathrm{Ba}$ and $\mathrm{Sr}$ ), the samples from two trends show systematically different trace element abundances. The order of incompatible elements is derived from Hofmann (1988) with Cs and all REE added. NMORB values after Sun and McDonough (1989).

Additional data for the monogenetic cones are from several sources (Churikova et al., 2001; Bryant et al., 2011; Volynets et al., 2013). 


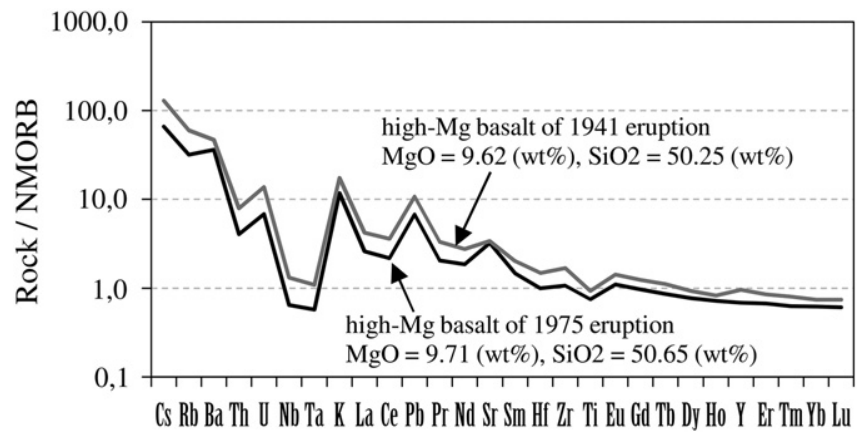

Fig. 12. Trace element distribution in high-Mg lavas of the 1975-1976 and 1941 eruptions normalized to NMORB.

1 kbar (Flerov et al., 1984). Using samples 655 and 201, we simulated trend 2 in a series of computations under dry conditions $(0-0.5 \%$ water content), NNO, QFM, IW oxygen buffers, and pressures of 110 kbar both under constant pressure and decompression. Both fractional and equilibrium crystallization were used to model trend 2. This approach replicated trend 2 for major element fields, including the samples from the Menyailov Vent, under water content of $0 \%$, NNO oxygen buffer, constant pressure 2 kbar with fractional crystallization (Fig. 14).

Recently it was shown by $\mathrm{H}_{2} \mathrm{O}$ measurements in melt inclusions from high-Mg olivines from sample of 1004 (an analog of the 1941 eruption) that the water content in an early olivine phase was up to 2.6 wt.\% (Portnyagin et al., 2007b). However, earlier experimental results suggest anhydrous conditions for crystallization of the entire mineral assemblage. These data and the existence of two olivine generations in high-Mg lavas (Flerov et al., 1980, 1984) testify that the high-K melts were degassed during their ascent prior to the main phase of crystallization.

The $\mathrm{K}_{2} \mathrm{O} / \mathrm{Na}_{2} \mathrm{O}$ ratio as well as the incompatible elements should not vary during early $\mathrm{Ol}-\mathrm{Cpx}$-fractionation and regression lines for that ratio should have a small angle for trend 1 . Trend 2 shows the double increase of $\mathrm{K}_{2} \mathrm{O} / \mathrm{Na}_{2} \mathrm{O}$ with fractionation. This is evidence for massive $\mathrm{Pl}$ crystallization in trend 2 samples in comparison with those of trend 1. The higher concentrations of $\mathrm{Na}_{2} \mathrm{O}, \mathrm{Ba}$, and $\mathrm{Sr}$ in $\mathrm{Pl}$ compared to $\mathrm{K}_{2} \mathrm{O}$ and $\mathrm{Rb}$, and the $\mathrm{REE}, \mathrm{K}_{2} \mathrm{O} / \mathrm{Na}_{2} \mathrm{O}$ and $\mathrm{Rb} \mathrm{b}_{\mathrm{n}} / \mathrm{Ba}_{\mathrm{n}}$ ratio increases and $\mathrm{Sr}$ anomaly decreases are all expected during the course of Pl crystallization. The result is that the $R b_{n} / B a_{n}$ ratio increases and becomes higher than 1 and the $\mathrm{Sr}$ anomaly is negative (Fig. 15). Trend 1 evolution retains a $\mathrm{Rb}_{\mathrm{n}} / \mathrm{Ba}_{\mathrm{n}}$ ratio $<1$ and Sr-maximum (Fig. 15a), whereas trend 2 evolution shows an increase from $\mathrm{Rb}_{\mathrm{n}} / \mathrm{Ba}_{\mathrm{n}}<1$ to $\mathrm{Rb}_{\mathrm{n}} / \mathrm{Ba}_{\mathrm{n}}>1$ and a drop from Sr-maximum to Sr-minimum (Fig. 15b and c). These calculations confirm the petrological observations that the high-K trachybasalts are rich in $\mathrm{Pl}$ and have negative $\mathrm{Sr}$ anomalies.

The major element compositions in samples 655 and 201 are close to each other (except $\mathrm{K}_{2} \mathrm{O}$ and $\mathrm{P}_{2} \mathrm{O}_{5}$ ) and thus do not allow us to discriminate between them as potential parental melts for trend 2 rocks. Modeling of $\mathrm{Rb}$ and $\mathrm{Yb}$ aligns well with rock compositions from trend 1 for sample 655 under hydrous conditions and with rocks compositions from trend 2 for sample 201 under anhydrous conditions (Fig. 16). At the same time results of modeling for sample 655 under anhydrous conditions have systematically underestimated concentrations of $\mathrm{Rb}$ and of $\mathrm{Yb}$. Thus modeling of trace element compositions allows us to select sample 201 as parental melt for rocks of trend 2 .

Our results are not strictly dependent on the choice of $\mathrm{P}-\mathrm{T}-\mathrm{H}_{2} \mathrm{O}-\mathrm{fO}_{2}$ conditions for differentiation of the Tolbachik massif magmas (Figs. 1416). Calculations that differ solely by water content emphasize the main difference between the two trends: hydrous and anhydrous conditions. Our natural data and calculated results are in line with the differentiation trends obtained experimentally for hydrous and anhydrous conditions for ferrobasaltic magmas (Botcharnikov et al., 2008).
Another important conclusion relates to the choice of parental melts. Sample 655 (high-Mg basalts of 1975-1976 Northern Breakthrough) is plotted on major element diagrams at the crossover position of both trends. In terms of major element calculations, both trends are well reproduced from sample 655 . However, the enhanced concentrations of trace elements in the trend 2 rocks cannot be explained by fractional crystallization from sample 655 . So, in terms of major and trace element calculations trend 1 is well reproduced from sample 655 , and trend 2 is well reproduced from sample 201. Based on this calculation we argue that sample 655 (high-Mg basalt of the 1975-1976 Northern Breakthrough) is a parental melt for trend 1 rocks and sample 201 (highMg basalt from the 1941 eruption) is a parental melt for trend 2 lavas. We also found no need to call upon magma mixing to explain any of the data.

\subsection{Isotope observations}

The samples with different major and trace element signatures show consistent variations in $\mathrm{Sr}-\mathrm{Nd}$ space. All cinder cone lavas, including high-Mg basalts of the 1941 eruption and 1975-1976 Northern Breakthrough, as well as high-K trachybasalts, plot close together with low $\mathrm{Sr}-$ and high Nd-isotope ratios. All stratovolcano and pedestal lavas have higher ${ }^{87} \mathrm{Sr} /{ }^{86} \mathrm{Sr}$ and similar or slightly lower ${ }^{143} \mathrm{Nd} /{ }^{144} \mathrm{Nd}$ ratios (Fig. 8). Even samples from Plosky Tolbachik stratovolcano and a dike (TOL-12-38 and TOL-12-50, respectively) that show high-K geochemical signatures of trend 2 in major and trace elements are more enriched in Sr-isotopes compared to the cones (Fig. 9).

These data are indicative of the fact that enrichment in $\mathrm{Sr}$ isotopes and accompanying slight depletion in Nd isotopes are not related to geochemical variations between trends 1 and 2, but were caused by processes that were ongoing within the crustal magma chamber prior to eruption. Although addition of subduction zone fluid could increase the radiogenic Sr, there is no systematic enrichment in fluid-mobile elements of the rocks from stratovolcano, pedestal, and dike complexes compared to the monogenetic cones.

Crustal contamination within the crustal magma chamber can account for the observed shift in $\mathrm{Sr}-\mathrm{Nd}$ isotope systematics. Overall ${ }^{87} \mathrm{Sr} /{ }^{86} \mathrm{Sr}$ in the lavas varies from 0.70333 to 0.70359 (Figs. 8, 9). The lowest values of $0.703343 \pm 6$ (the average for 4 samples: three from cinder cones and one summit lava) indicate a negligible contribution of crustal material to the mantle-related parental magmas and are characteristic of the monogenetic basalts. In the samples from the

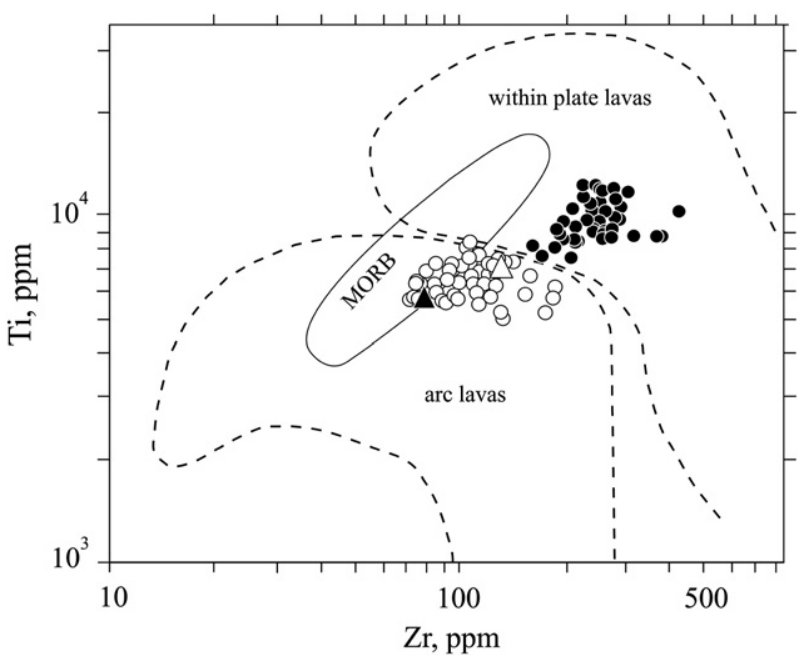

Fig. 13. Concentration of Ti versus Zr (after Pearce, 1982) showing the fields of volcanicarc basalts, MORB and within-plate basalts (note logarithmic scales). While all samples from trend 1 plot inside the field of volcanic-arc basalts, all samples from trend 2 (except 1941 eruption) plot inside the field of within-plate basalts. Symbols are the same as for Fig. 7. 

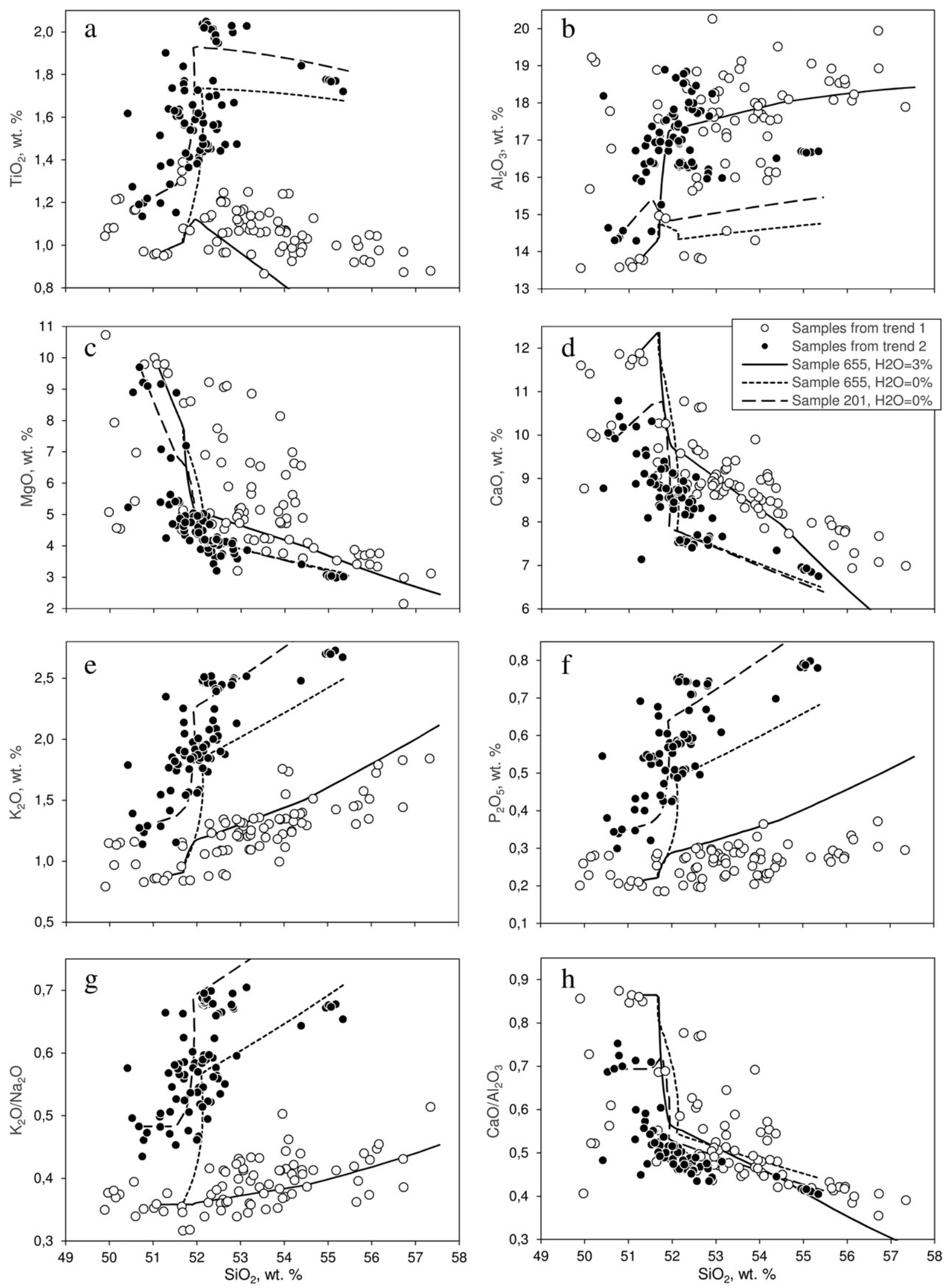

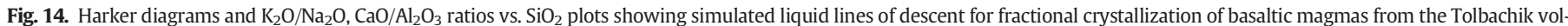

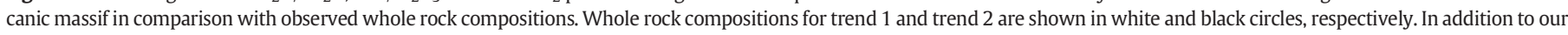

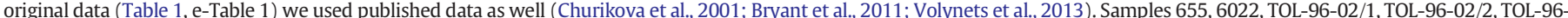

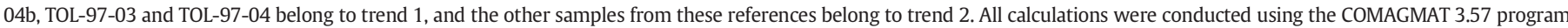

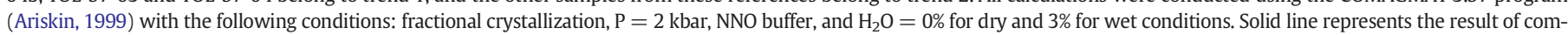

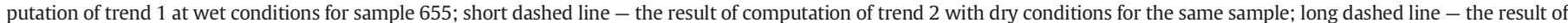
computation of trend 2 with dry conditions for sample 201. 

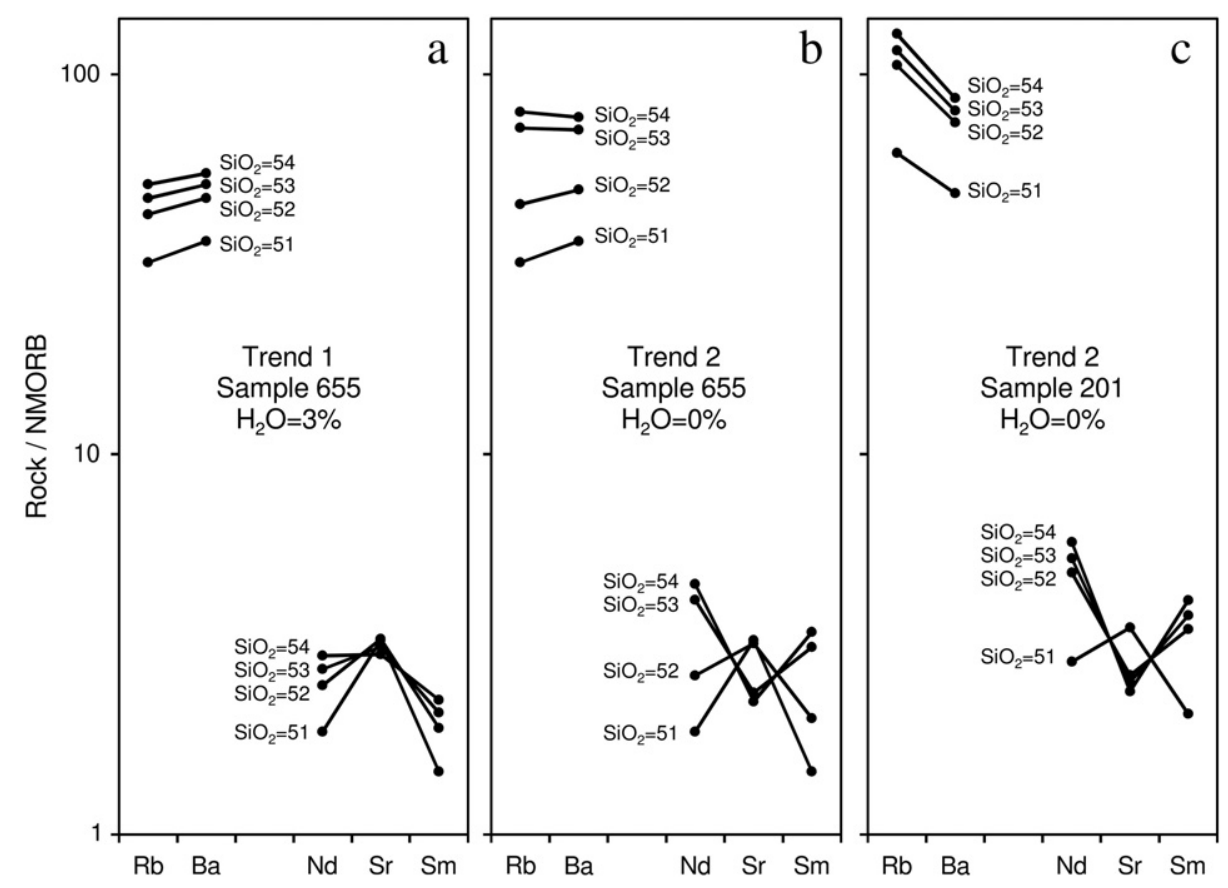

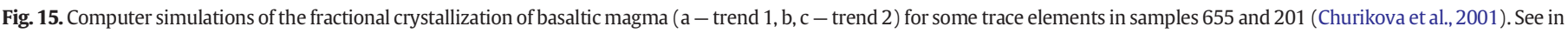

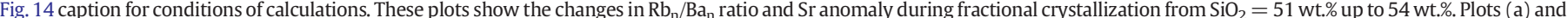

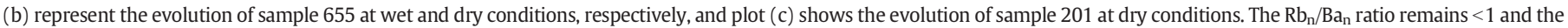

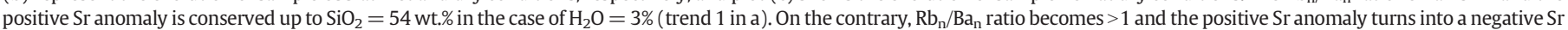

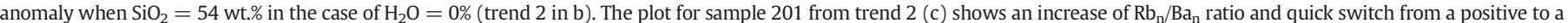
negative $\mathrm{Sr}$ anomaly.

stratovolcanoes, pedestal and dikes this ratio is higher $(0.703474 \pm 8$ : the average for 7 samples), and are the highest for Mount Povorotnaya $(0.703571 \pm 7$ : the average for 2 samples) (Table 2, Fig. 8). The enrichment in Th/Ta (Fig. 7d), Th/Nb and La/Sm (Fig. 7f) ratios in the rocks of periods 1-3 compared to Holocene lavas also is consistent with the above assumption.

AFC model was considered for the interpretation of these results. In our model the initial melt has $340 \mathrm{ppm} \mathrm{Sr}$ with ${ }^{87} \mathrm{Sr} /{ }^{86} \mathrm{Sr}=0.70333$, which corresponds to average values for the cinder cones lavas. Although pre-Quaternary basement rocks have not been reported for the KGV area, the geological setting as well as geophysical data of the area indicates that Paleozoic crystalline basement is represented by the Kamchatskaya and the Kolpakovskaya series. Rocks of these series are mainly schists and gneisses with an average $\mathrm{Sr}$ concentrations of $240 \mathrm{ppm}$ and ${ }^{87} \mathrm{Sr} /{ }^{86} \mathrm{Sr}=0.7070$ (Vinogradov et al., 1988, 1991). As these rocks are not characterized by Nd isotopes, we only used $\mathrm{Sr}$ isotope data for the AFC model.

The relationship between $\mathrm{Sr}$ isotope compositions and $\mathrm{Sr}$ concentrations for Tolbachik rocks and the model calculations for simultaneous assimilation and fractional crystallization (AFC model of DePaolo, 1981) are shown in Fig. 17. The analytical results are compatible with the model if we assume that not all crystals have been removed from the liquid: the compositional changes to the crystals and melts predicted by the model are shown by solid lines (Fig. 17). The calculated ratio (r) of the mass assimilated to the mass crystallized is 0.2 . The bulk distribution coefficient is $D_{S r}=3.5$, as deduced from the average ratio of phenocrysts in volcanic rocks: Pl:Ol:Aug:Mt = 75:10:10:5. The mineral distribution coefficients are from Henderson (1982). The amount of assimilated material is shown to the right of the crystal evolution line in Fig. 17.

Our simple calculations suggest the involvement of about $2 \%$ crustal contamination in the petrogenesis of the stratovolcano, pedestal, and dike complexes and about 3-4\% contamination for the Mount Povorotnaya rocks. High $\delta^{18} \mathrm{O}\left(7.1 \%\right.$ ) and high ${ }^{187} \mathrm{Re} /{ }^{188} \mathrm{Os}$ (five times higher in a single Plosky Tolbachik stratovolcano sample than in monogenetic cones; Alves et al., 1999; Pineau et al., 1999) may also be also the result of crustal assimilation processes. These data are consistent with the occurrence of crustal xenoliths in Ostry Tolbachik stratovolcano (Flerov et al., 2015).

\subsection{Menyailov Vent and KGV basement - similarities and differences}

High-K rocks are rather rare in Kamchatka and inside the CKD are found only in the Ploskye Sopki and Tolbachik massifs and Nikolka volcano, although only limited data exist for Nikolka volcano. The origin of the high-K rocks is still under debate. We analyzed six samples from the KGV basement of basalt-basaltic andesite composition centered on the Ploskye Sopki volcanic massif (Tables 1, 3, and e-Table 1). These samples represent high-K lavas identical to other high-K basalts and basaltic andesites from Ploskye Sopki massif in major and trace elements (Figs. 4, 5 and 11 in Churikova et al., 2015).

As shown earlier, KGV basement rocks fall close to the Menyailov Vent samples in most of the major element diagrams (Figs. 4, 5) suggesting a similar origin for both sets of samples. Most trace element ratios in the lavas of trend 2 and KGV basement are also similar (Fig. 7a, b, e).

But some differences in trace element distributions and isotopic ratios exist. The $\mathrm{Rb} / \mathrm{Ba}_{\mathrm{n}}$ ratio in lavas of the Menyailov Vent is greater than 1 , while in the KGV plateau basalts $\mathrm{Rb}_{\mathrm{n}} / \mathrm{Ba}_{\mathrm{n}}<1$ at nearly the same $\mathrm{Sr}$ concentrations (Fig. 18a). High-K rocks from Ploskye Sopki are higher in ${ }^{87} \mathrm{Sr} /{ }^{86} \mathrm{Sr}$ compared to high-K lavas from Tolbachik. Moreover, high-K rocks from Ploskye Sopki are enriched in radiogenic $\mathrm{Sr}$ compared to middle-K rocks, while the high-K lavas from Tolbachik are depleted in ${ }^{87} \mathrm{Sr} /{ }^{86} \mathrm{Sr}$ compared to the middle-K ones (Fig. 18b).

Based on these data we conclude that the high-K rock series on different KGV volcanoes may not be genetically related. Churikova (1993) showed that fractional crystallization at hydrous and anhydrous conditions could account for rock variations at Ploskye Sopki massif. In this case the difference in high-K rock geochemistry could reflect the difference in parental melt compositions underneath both massifs. 

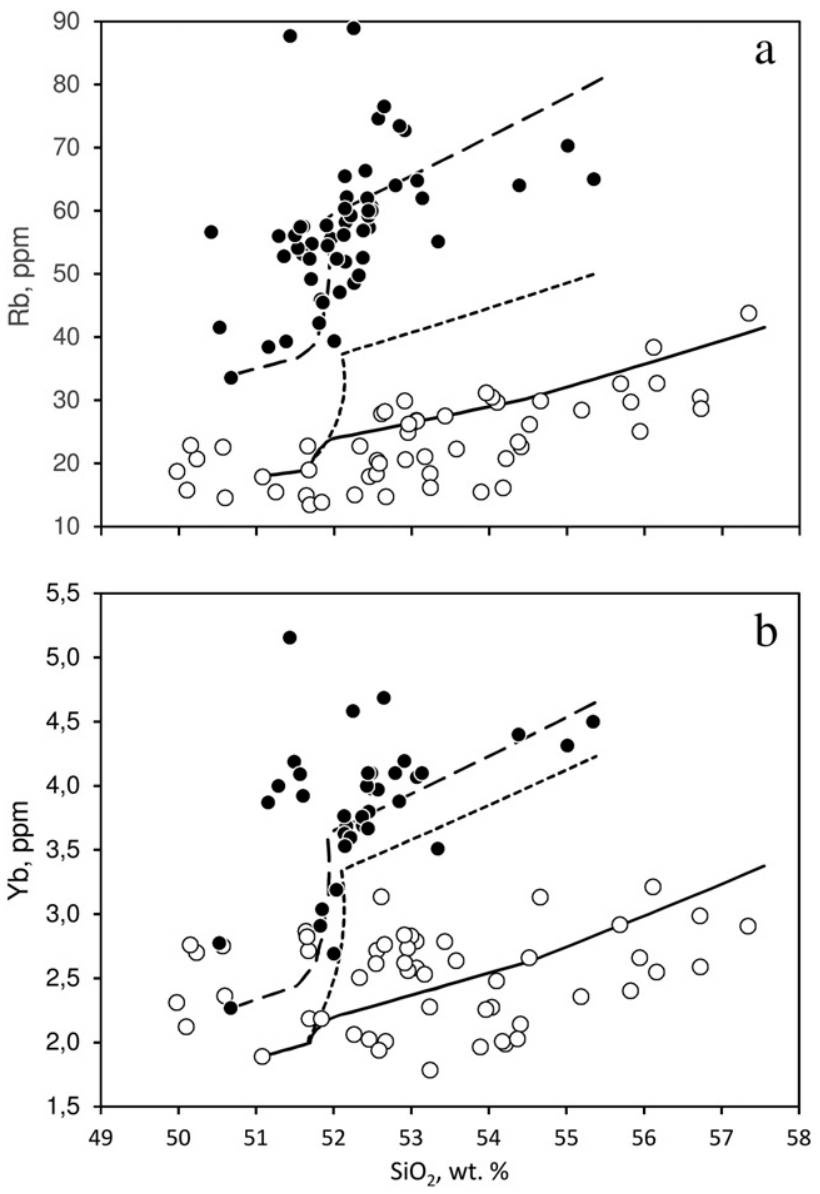

Fig. 16. Computer simulations of the fractional crystallization of basaltic magma for $\mathrm{Rb}$ (a) and $\mathrm{Yb}$ (b) in samples 655 and 201 (Churikova et al., 2001). The symbols and the conditions of calculations are the same as in Fig. 14. As addition to our ICP-MS data, we used the trace element data from Churikova et al. (2001), Bryant et al. (2011), and Volynets et al. (2013). Based on the trace element distributions, samples 655 and 6022 belong to trend 1 , while other samples from these references belong to trend 2 . The computational results reasonably reproduce the data from sample 655 for wet conditions and from sample 201 for dry conditions. The computational results for sample 655 for dry conditions are systematically understated.

\subsection{Origin of Mount Povorotnaya}

Mount Povorotnaya is a separate edifice on the northeastern slope of the Tolbachik volcanic massif (Fig. 3a), located 8 km from Plosky Tolbachik summit. Its origin has always been subject to disputes. Ermakov and Vazheevskaya (1973) considered this edifice to be a block of the Tolbachik volcano pedestal, while others suggested that it was a large toreva block-an intact fragment of the collapsed volcanic edifice (Reiche, 1937)-that slid down from the top of Plosky Tolbachik stratovolcano about $6500 \mathrm{yr} \mathrm{BP}\left({ }^{14} \mathrm{C}\right.$ ) (Melekestsev and Braitseva, 1984; Ponomareva et al., 2006).

Our field observations show that the edifice of Mount Povorotnaya has no stratovolcano-like structure and consists of two different rock suites that show clear misalignment. Lava flows in the lower part of Mount Povorotnaya have angles of inclination about $30^{\circ}$ to the W-SW, while the upper block has a sub-horizontal bedding. In this lower part augite-phyric rocks are abundant. The lavas of the upper part are more enriched in $\mathrm{Pl}$ and $\mathrm{Ol}$, but still contain $\mathrm{Cpx}$ as the main phenocryst with large phenocrysts up to $0.5-1 \mathrm{~cm}$. Such Cpx-phyric rocks were also frequently found in the Tolbachik pedestal complex, but were never found in other complexes of the Tolbachik massif.

Geochemical features of the Mount Povorotnaya rocks are shown in Figs. 4, 5, 18c. For major elements, all lavas of Mount Povorotnaya fall

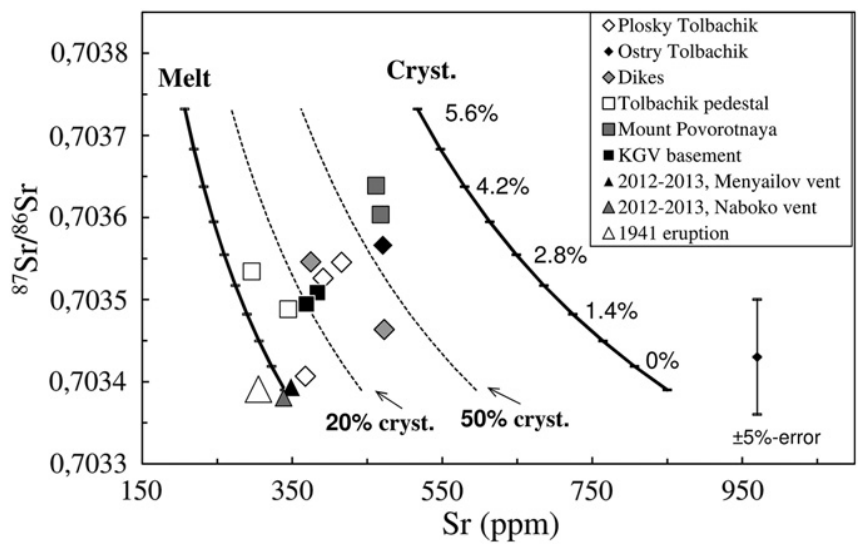

Fig. 17. Results from AFC modeling of whole rock compositions from the Tolbachik volcanic massif. Symbols are the same as for Fig. 2. Solid lines - calculated trajectories for the evolution of melt and crystals; the amount of assimilated material is shown as percentages to the right of the line of crystal evolution. Dotted lines - calculated trajectories with $20 \%$ and $50 \%$ crystals in the melt. See the text for details.

within trend 1. All samples are significantly evolved (52.7-55.7 wt.\% $\mathrm{SiO}_{2}$ ) and have a range of $\mathrm{Mg \#} \mathrm{(43-60).} \mathrm{The} \mathrm{distribution} \mathrm{of} \mathrm{trace} \mathrm{ele-}$ ments in Mount Povorotnaya rocks is also similar to that of the pedestal samples with rather depleted trace element patterns and low HFSE (Fig. 18c). But in contrast to pedestal samples, Mount Povorotnaya lavas are enriched in LILE such as $\mathrm{Cs}, \mathrm{Ba}, \mathrm{Pb}$ and $\mathrm{Sr}$ (Fig. 18c) suggesting a higher amount of crustal material in their melts. In $\mathrm{Sr}-\mathrm{Nd}$ isotope systematics, Mount Povorotnaya lavas are the most enriched in ${ }^{87} \mathrm{Sr} /{ }^{86} \mathrm{Sr}$ and depleted in ${ }^{143} \mathrm{Nd} /{ }^{144} \mathrm{Nd}$, suggesting some more crustal material in their melts compared to other Tolbachik rocks (Figs. 8, 9).

Our petrological and geochemical data suggest that Mount Povorotnaya cannot be a toreva block of Plosky Tolbachik volcano, but is a block of the Tolbachik pedestal. These data are consistent by $\mathrm{K}-\mathrm{Ag}$ dating. Mount Povorotnaya, with the age of $0.306 \pm 0.01 \mathrm{Ma}$, is not only older than the pedestal rocks of the Tolbachik volcanic massif in vicinity of this volcano $(0.086 \pm 0.016 \mathrm{Ma})$, but is the oldest isotopically dated edifice within the entire KGV.

\subsection{Formation of the Tolbachik volcanic massif}

Based on our new data and analysis from all of the different magmatic components of the Tolbachik volcanic massif, we can construct a history for the geochemical evolution of the massif as follows:

In Middle-Late Pleistocene, the pedestal of Tolbachik volcanic massif began to form. At that time, only one rock series, referred to here as trend 1, was active, producing depleted high-Mg (Mg\# 43-64) and low-viscosity lavas, resulting in formation of a low, sloping shield volcano. The rocks of trend 1 continued to be erupted during the first stages of stratovolcano formation. During these periods more evolved (Mg\# 37-55) lavas were erupted. The viscosity of stratigraphically lower stratovolcano lavas was higher compared to that of the pedestal lavas, resulting in steeper slopes with inclinations of up to $20-30^{\circ}$. According to our data both Ostry Tolbachik and Plosky Tolbachik stratovolcanoes have had a very similar geochemical evolution in terms of major and trace elements. During formation of the pedestal, the lower parts of stratovolcanoes and the dikes, the parental melts were stored at relatively shallow depth within a crustal magma chamber. The upper mantle layers, depleted relative to N-MORB, supplied middle-K melts to the same chamber. The middle- $K$ melts with island arc characteristics fractionated at water-rich conditions, partially assimilating crustal material from the chamber walls. The rocks of these periods clearly form trend 1 on major and trace element diagrams and show small amounts of crustal assimilation on isotopic plots.

At the Late Pleistocene-Holocene boundary, the geodynamic regime changed resulting in the opening of a large fissure zone. The 

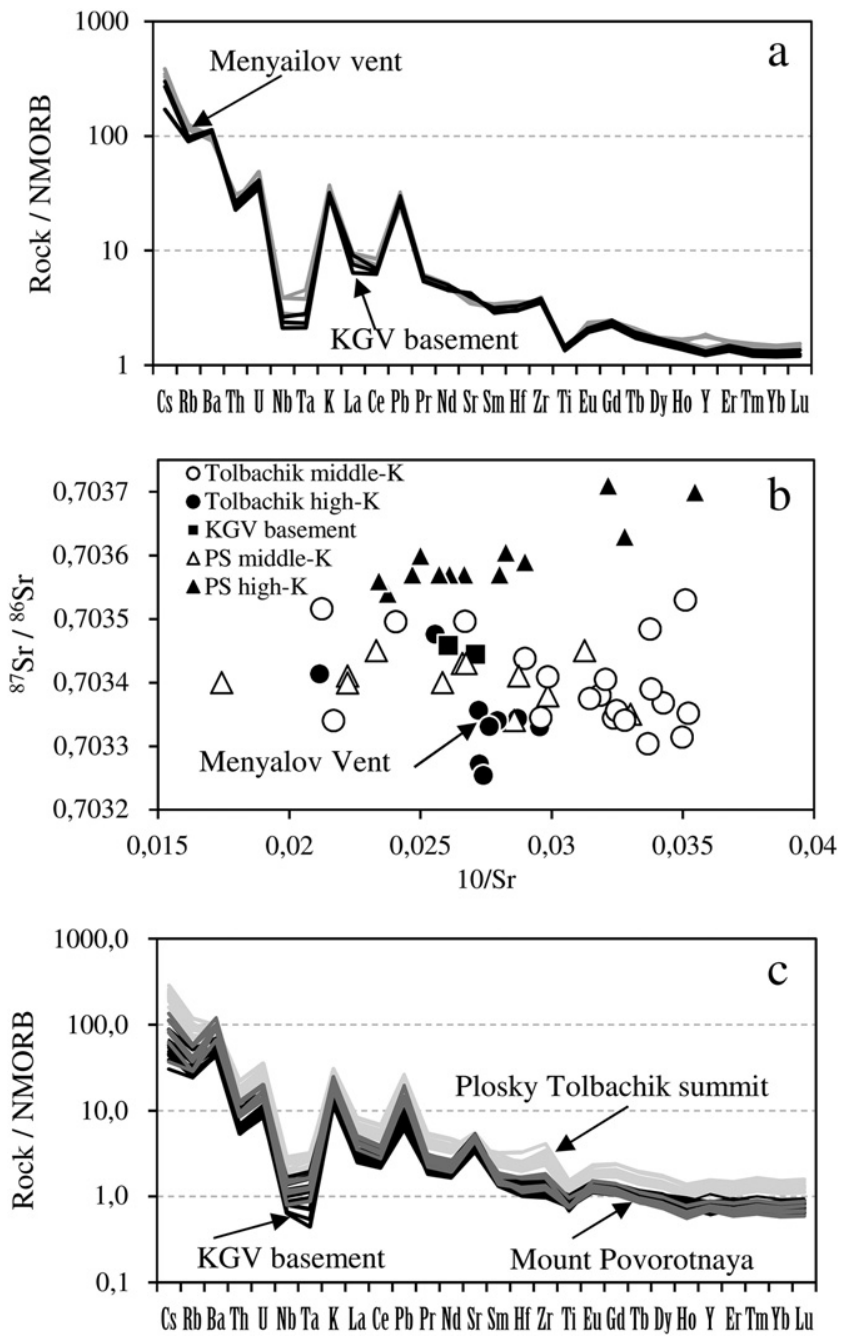

Fig. 18. Geochemical features of the Menyailov Vent, Mount Povorotnaya and KGV basement: (a) trace element distribution in lavas of the Menyailov Vent (black lines) and KGV basement (gray lines). Additional data for the Menyailov Vent are from Volynets et al. (2013); (b) ${ }^{87} \mathrm{Sr} /{ }^{86} \mathrm{Sr}$ vs. $10 / \mathrm{Sr}$ in rocks from the Tolbachik and Ploskye Sopki massifs. Additional data are from Churikova and Sokolov (1993) and Portnyagin et al. (2007a); (c) trace element compositions in Mount Povorotnaya rocks. Geochemically the rocks of Mount Povorotnaya fall into the area of pedestal rocks of the Tolbachik volcanic massif with enrichment in fluid-mobile elements. The rocks of the Plosky Tolbachik summit are given for the comparison.

accompanying extension and upwelling, a result of intra-arc rifting, produced high-K melts, not depleted relative to N-MORB, but with island arc signatures and rift-like characteristics. Fissure eruptions allowed for degassing of the melts at shallow depth, and parental melts fractionated at anhydrous conditions as opposed to magmas that produced the rocks of trend 1 , which fractionated at water-rich conditions. These high-K rocks contributed to the formation of the upper parts of stratovolcanoes. Such rocks were enriched in $\mathrm{K}, \mathrm{P}$, Ti, and all incompatible trace elements, and clearly form trend 2 on major and trace element diagrams. The rocks of trend 2 , which form the upper parts of the stratovolcanoes, were also affected by crustal assimilation, but to a lesser extent compared to samples from trend 1 . The first high-K rocks were found at an elevation of 1400 masl on Plosky Tolbachik and at an elevation of 2000 masl on Ostry Tolbachik. As the pedestal lavas formed the edifice up to elevations of 1000-1500 masl (Ermakov and Vazheevskaya, 1973), this means that the high-K rocks of trend 2 started to erupt approximately at the beginning of stratovolcano formation. The change of geodynamic regime was not instantaneous, as confirmed by intermittent lavas of both trends in the upper parts of the stratovolcanoes and by the existence of numerous dikes generated by both magma series, which were the feeding channels to stratovolcano lavas. During the first stages of stratovolcano formation, the lavas of trend 1 were predominant, whereas during the formation of the upper parts of both stratovolcanoes the lavas of trend 2 became prevalent.

At the beginning of Holocene, the last deglaciation in KGV enhanced decompression effect (Sigvaldson et al., 1992). During this time the majority of monogenetic cones along the $70 \mathrm{~km}$ long fissure zone and the Hawaiian-type pit crater/caldera with the diameter of about $3 \mathrm{~km}$ at the Plosky Tolbachik summit were formed. The rocks of trend 2 became prevalent during this period and formed cinder cone-lava fields along the fissure zone. It is possible that the parental melts of this period were not resident in a crustal magma chamber or the residence time in the chamber was very short, thus, the parental melts of trend 2 rocks were rising to the surface faster thus avoiding crustal assimilation. The prevalence of the high-K lavas has remained during the period of monogenetic cones formation up to the recent time. And yet, the existence of several cones of both old and young age with geochemical characteristics of lavas from trend 1 (e.g. high-Mg basalts of 19751976 Northern Breakthrough) suggests that middle-K parental melts still exist.

\section{Conclusions}

(1) A wide ranging investigation of the Tolbachik volcanic massif with sampling of its different complexes, such as pedestal, stratovolcanoes, dikes, cinder cone-lava fields, and lava flows has identified the presence of middle-K (trend 1 ) and high-K (trend 2 ) rock series with differences in major and trace elements, and trace element ratios.

(2) We attribute the occurrence of the trend 2 rocks at Tolbachik massif to a change in the geodynamic conditions in this area at the Late Pleistocene-Holocene boundary resulting in the establishment of a fissure system, upwelling as a result of intra-arc rifting, and degassing of melts at shallow depths.

(3) As a result of melts degassing, the conditions of crystallization changed from water-rich for trend 1 lavas to anhydrous for trend 2 rocks. This shows that crystallization at different water activities can be one of the important processes responsible for variations in rock compositions at the Tolbachik volcanic massif.

(4) Mantle upwelling due to extension contributed to the enhanced trace element abundances in melts of trend 2. Variable depletion of the same mantle source can explain all trace element variations at Tolbachik volcanic massif.

(5) The enrichment of the pedestal, stratovolcano and dike complexes in radiogenic $\mathrm{Sr}$ and depletion in $\mathrm{Nd}$-isotopes is explained by low percentage ( $2-4 \%$ ) crustal assimilation, but the lavas from monogenetic cones do not show evidence for crustal assimilation. The magma storage conditions prior to eruption may be the main contributing factor for the addition of crustal material.

(6) The rocks of Mount Povorotnaya are similar in petrography and chemical composition to those of the Tolbachik massif pedestal. Based on $\mathrm{K}-\mathrm{Ar}$ dating, we conclude that the Mount Povorotnaya is an old block of the Tolbachik massif pedestal and for the moment the oldest known volcanic feature in Klyuchevskaya group of volcanoes.

Supplementary data to this article can be found online at http://dx. doi.org/10.1016/j.jvolgeores.2015.10.026.

\section{Acknowledgments}

We are very grateful to Roman Botcharnikov and an anonymous reviewer for their useful comments on an earlier version of the manuscript. The authors thank colleagues from the IViS FEB RAS who helped 
us in organization of field works: O.E. Bograd, Yu.V. Demyanchuk, N.P. Egorova, Ya.D. Muraviev, and V.P. Fedulova. We appreciate contribution of our selfless volunteers who worked with us on the Tolbachik volcanic massif: M.M. Yarin, E.I. Chesalova, K.D. Gorokhov, E.A. Nikonova, A.Ya. Muraviev, D.V. Okopny, and A.V. Kolchina. We also thank G.B. Flerov who provided us with some samples of 2012-2013 eruption. We had very useful discussions with G.B. Flerov, I.V. Melekestsev, and P.E. Izbekov. Our sincere appreciation to E.L. Rossovskaya and B. Edwards for their language improvements of the manuscript. This research was supported by RFBR grant \# 08-05-00600 and RFBR-JSPS grant \# 13-05-92104.

\section{References}

Almeev, R.R., Kimura, J.-I., Ariskin, A.A., Ozerov, A.Y., 2013. Decoding crystal fractionation in calc-alkaline magmas from the Bezymianny Volcano (Kamchatka, Russia) using mineral and bulk rock compositions. J. Volcanol. Geotherm. Res. 263, 141-171.

Alves, S., Schiano, P., Allègre, C.J., 1999. Rhenium-osmium isotopic investigation of Java subduction zone lavas. Earth Planet. Sci. Lett. 168 (1-2), 65-77.

Ariskin, A.A., 1999. Phase equilibria modeling in igneous petrology: use of COMAGMAT model for simulating fractionation of ferro-basaltic magmas and the genesis of high-alumina basalt. J. Volcanol. Geotherm. Res. 90 (1-2), 115-162.

Bindeman, I.N., Davis, A.M., 2000. Trace element partitioning between plagioclase and melt: investigation of dopant influence on partition behavior. Geochim. Cosmochim. Acta 64 (16), 2863-2878.

Bindeman, I.N., Ponomareva, V.V., Bailey, J.C., Valley, J.W., 2004. Volcanic arc of Kamchatka: a province with high- $\delta^{18} \mathrm{O}$ magma sources and large-scale ${ }^{18} \mathrm{O} /{ }^{16} \mathrm{O}$ depletion of the upper crust. Geochim. Cosmochim. Acta 68 (4), 841-865.

Bindeman, I.N., Eiler, J.M., Yogodzinski, G.M., Tatsumi, Y., Stern, C.R., Grove, T.L., Portnyagin, M., Hoernle, K., Danyushevsky, L.V., 2005. Oxygen isotope evidence for slab melting in modern and ancient subduction zones. Earth Planet. Sci. Lett. 235 (3-4), 480-496.

Botcharnikov, R.E., Almeev, R.R., Koepke, J., Holtz, F., 2008. Phase relations and liquid lines of descent in hydrous ferrobasalt - implications for the Skaergaard Intrusion and Columbia River Flood Basalts. J. Petrol. 49 (9), 1687-1727.

Bryant, J.A., Yogodzinski, G.M., Churikova, T.G., 2011. High-Mg\# andesitic lavas of the Shisheisky Complex, Northern Kamchatka: implications for primitive calc-alkaline magmatism. Contrib. Mineral. Petrol. 161 (5), 791-810.

Calkins, J.A., 2004. ${ }^{40} \mathrm{Ar} /{ }^{39} \mathrm{Ar}$ geochronology of Khapitsa plateau and Studyonaya River basalts and basaltic andesites in Central Kamchatka Depression, Kamchatka, Russia. In: Gordeev, E.I. (Ed.), Linkages Among Tectonics, Seismicity, Magma Genesis, and Eruption in Volcanic Arcs. IV International Biennial Workshop on Subduction Processes Emphasizing the Japan-Kurile-Kamchatka-Aleutian Arcs. August 21-27, 2004. Institute of Volcanology and Seismology FEB RAS, Petropavlovsk-Kamchatsky, pp. 53-54 (http://www.kscnet.ru/ivs/conferences/kasp/tez/ab21en.doc).

Churikova, T.G., 1993. Geochemistry and Modeling of the Magmatic Processes of the Klyuchevskaya Group of Volcanoes PhD thesis Lomonosov Moscow State University, Moscow (155 pp., (in Russian). http://www.dissercat.com/content/geokhimiya-imodelirovanie-magmaticheskogo-protsessa-vulkanov-klyuchevskoi-gruppy).

Churikova, T.G., Sokolov, S.Y., 1993. The magmatic evolution of Ploskye Sopki volcano, Kamchatka (analyses of Sr isotopic geochemistry). Geokhimiya 10, 1439-1448 (In Russian).

Churikova, T., Dorendorf, F., Wörner, G., 2001. Sources and fluids in the mantle wedge below Kamchatka, evidence from across-arc geochemical variation. J. Petrol. 42 (8), 1567-1593.

Churikova, T., Wörner, G., Mironov, N., Kronz, A., 2007. Volatile (S, Cl and F) and fluid mobile trace element compositions in melt inclusions: implications for variable fluid sources across the Kamchatka arc. Contrib. Mineral. Petrol. 154 (2), 217-239.

Churikova, T.G., Gordeychik, B.N., Ivanov, B.V., Wörner, G., 2013. Relationship between Kamen Volcano and the Klyuchevskaya group of volcanoes (Kamchatka). J. Volcanol. Geotherm. Res. 263, 3-21.

Churikova, T.G., Gordeychik, B.N., Edwards, B., Ponomareva, V.V., Zelenin, E.A., 2015. The Tolbachik volcanic massif: a review of the petrology, volcanology and eruption history prior to the 2012-2013 eruption. J. Volcanol. Geotherm. Res. 307, 3-21.

DePaolo, D.J., 1981. Trace element and isotopic effects of combined wallrock assimilation and fractional crystallization. Earth Planet. Sci. Lett. 53 (2), 189-202.

Dorendorf, F., Wiechert, U., Wörner, G., 2000. Hydrated sub-arc mantle: a source for the Kluchevskoy volcano, Kamchatka/Russia. Earth Planet. Sci. Lett. 175 (1-2), 69-86.

Dosseto, A., Bourdon, B., Joron, J.-L., Dupré, B., 2003. U-TH-PA-RA study of the Kamchatka arc: new constraints on the genesis of arc lavas. Geochim. Cosmochim. Acta 67 (15), 2857-2877.

Erlich, E.N., Gorshkov, G.S. (Eds.), 1979. Quaternary volcanism and tectonics in Kamchatka. Bulletin Volcanologique 42(1-4), pp. 1-298.

Ermakov, V.A., 1977. Stratigraphic Subdivision of the Quaternary Volcanic Rocks. Nedra, Moscow (223 pp. (in Russian)).

Ermakov, V.A., Vazheevskaya, A.A., 1973. Ostry and Plosky Tolbachik volcanoes. Biull. Vulkanol. Stantsii 49, 43-53 (In Russian).

Fedotov, S.A. (Ed.), 1984. The Large Fissure Tolbachik Eruption: Kamchatka 1975-1976. Nauka, Moscow (637 pp. (In Russian))

Fedotov, S.A., Markhinin, Y.K. (Eds.), 1983. The Great Tolbachik Fissure Eruption. Geological and Geophysical Data 1975-1976. Cambridge University Press, Cambridge (353 pp.).
Flerov, G.B., Melekestsev, I.V., 2013. Eruption 2012-2013 as a result of the ongoing activity of Tolbachik regional zone of cinder cones (Klyuchevskaya group of volcanoes, Kamchatka). In: Gordeev, E.I. (Ed.), Volcanism and Associated Processes. Regional Scientific Conference Dedicated to the Day of Volcanologist. March 29-30, 2013. Institute of Volcanology and Seismology FEB RAS, Petropavlovsk-Kamchatsky, pp. 139-144 ((In Russian). http://www.kscnet.ru/ivs/publication/volc_day/2013/art20.pdf).

Flerov, G.B., Ovsyannikov, A.A., 1991. Ushkovsky volcano. In: Fedotov, S.A., Masurenkov, Y.P. (Eds.), Active Volcanoes of Kamchatka 1. Nauka, Moscow, pp. 164-165.

Flerov, G.B., Sobolev, A.V., Koloskov, A.V., 1980. Pyroxenes, olivines and spinels in the rocks of Grate Fissure Tolbachik Eruption. Vulkanol. Seisol. 3, 3-15 (In Russian)

Flerov, G.B., Andreev, V.N., Budnikov, V.A., Tsyurupa, A.I., 1984. Petrology of the eruption products. In: Fedotov, S.A. (Ed.), The Large Fissure Tolbachik Eruption: Kamchatka 1975-1976. Nauka, Moscow, pp. 223-284 (In Russian).

Flerov, G.B., Anan'ev, V.V., Ponomarev, G.P., 2015. The petrogenesis of rocks of the Ostry and Ploskii volcanoes and the relationship between volcanic occurrences of basaltic and trachybasaltic magmas in the Tolbachik Dol Area, Kamchatka. J. Volcanol. Seismol. 9 (3), 162-181. http://dx.doi.org/10.1134/S0742046315030021.

Henderson, P., 1982. Inorganic Geochemistry. Pergamon Press, Oxford, p. 353.

Hirahara, Y., Takahashi, T., Miyazaki, T., Vaglarov, B.S., Chang, Q., Kimura, J.-I., Tatsumi, Y., 2009. Precise Nd isotope analysis of igneous rocks using cation exchange chromatography and thermal ionization mass spectrometry (TIMS). JAMSTEC Report of Research and Development Special Issue: IFREE Technical Report, pp. 65-71 (http:// www.godac.jamstec.go.jp/catalog/data/doc_catalog/media/JAM_RandDsp_09.pdf).

Hochstaedter, A.G., Kepezhinskas, P., Defant, M., Drummond, M., Koloskov, A., 1996. Insights into the volcanic arc mantle wedge from magnesian lavas from the Kamchatka arc. J. Geophys. Res. Solid Earth 101 (B1), 697-712.

Hofmann, A.W., 1988. Chemical differentiation of the Earth; the relationship between mantle, continental crust, and oceanic crust. Earth Planet. Sci. Lett. 90, 297-314

Ivanov, B.V., Khrenov, A.P., 1979. The state of the craters of active volcanoes of Kamchatka in 1977-1978. Vulkanol. Seisol. 1, 97-101 (In Russian).

Kepezhinskas, P., McDermott, F., Defant, M.J., Hochstaedter, A., Drummond, M.S. Hawkesworth, C.J., Koloskov, A., Maury, R.C., Bellon, H., 1997. Trace element and $\mathrm{Sr}-\mathrm{Nd}-\mathrm{Pb}$ isotopic constraints on a three-component model of Kamchatka Arc petrogenesis. Geochim. Cosmochim. Acta 61 (3), 577-600.

Kersting, A.B., 1995. Pb isotope ratios of North Pacific sediments, sites 881, 883, and 884 implications for sediment recycling in the Kamchatkan Arc. Proc. Ocean Drill. Program Sci. Results 145, 383-388. http://dx.doi.org/10.2973/odp.proc.sr.145.152.1995.

Kersting, A.B., Arculus, R.J., 1994. Klyuchevskoy Volcano, Kamchatka, Russia: the role of high-fluxed, recharged, tapped and fractionated magma chamber(s) in the genesis of high- $\mathrm{Al}_{2} \mathrm{O}_{3}$ basalt from high-MgO basalt. J. Petrol. 35 (1), 1-42.

Kersting, A.B., Arculus, R.J., 1995. Pb isotope composition of Klyuchevskoy volcano, Kamchatka and North Pacific sediments: implications for magma genesis and crustal recycling in the Kamchatkan arc. Earth Planet. Sci. Lett. 136 (3-4), 133-148.

Khrenov, A.P., Dvigalo, V.N., Kirsanov, I.T., Fedotov, S.A., Gorelchik, V.I., Zharinov, N.A. 1991. Klyuchevskoy Volcano. In: Fedotov, S.A., Masurenkov, Y.P. (Eds.), Active Volcanoes of Kamchatka 1. Nauka, Moscow, pp. 146-153.

Kirsanov, I.T., Ponomarev, G.P., 1974. The eruption of Posky Tolbachik volcano and some of the features of its products. Biull. Vulkanol. Stantsii 50, 53-63 (In Russian).

Krivenko, A.P. (Ed.), 1990. Geochemical Types of Magmatic and Metamorphic Rocks of Kamchatka. Institute of Geology and Geophysics SB AN USSR, Novosibirsk (259 pp. (In Russian)).

Le Maitre, R.W., Streckeisen, A., Zanettin, B., Le Bas, M.J., Bonin, B., Bateman, P. (Eds.) 2002. Igneous Rocks: A Classification and Glossary of Terms: Recommendations of the International Union of Geological Sciences Subcommission on the Systematics of Igneous Rocks. Cambridge University Press (252 pp.).

Lukanin, O.A., Kadik, A.A., Biggar, G.M., Fedotov, S.A., 1980. Physico-chemical conditions of crystallization of the basalts of Grate fissure Tolbachik eruption 1975-1976. Vulkanol. Seisol. 3, 16-50.

Matsumoto, A., 1989. Improvement for determination of potassium in $\mathrm{K}-\mathrm{Ar}$ dating. Bull. Geol. Surv. Jpn. 40 (2), 65-70 (In Japanese).

Melekestsev, I.V., Braitseva, O.A., 1984. Gigantic rockslide avalanches on volcanoes. Volcanol. Seismol. 6, 495-508.

Melekestsev, I.V., Braitseva, O.A., Erlich, E.N., Kozhemyaka, N.N., 1974. Volcanic mountains and plains. In: Luchitsky, I.V. (Ed.), Kamchatka, Kurile and Commander Islands. Nauka, Moscow, pp. 162-234 (In Russian)

Melekestsev, I.V., Volynets, O.N., Ermakov, V.A., Kirsanova, T.P., Masurenkov, Yu.P., 1991 Sheveluch Volcano. In: Fedotov, S.A., Masurenkov, Y.P. (Eds.), Active Volcanoes of Kamchatka 1. Nauka, Moscow, pp. 98-103.

Menyailov, A.A., 1953. The state of Tolbachik volcano in 1946-1948. Biull. Vulkanol. Stantsii 17, 41-45.

Mironov, N.L., Portnyagin, M.V., Pletchov, P.Y., Khubunaya, S.A., 2001. Final stages of magma evolution in Klyuchevskoy Volcano, Kamchatka: evidence from melt inclusions in minerals of high-alumina basalts. Petrology 9 (1), 46-62.

Miyashiro, A., 1974. Volcanic rock series in island arcs and active continental margins. Am. J. Sci. 274 (4), 321-355.

Miyazaki, T., Vaglarov, B.S., Takei, M., Suzuki, M., Suzuki, H., Ohsawa, K., Chang, Q., Takahashi, T., Hirahara, Y., Hanyu, T., Kimura, J.-I., Tatsumi, Y., 2012. Development of a fully automated open-column chemical-separation system - COLUMNSPIDER - and its application to $\mathrm{Sr}-\mathrm{Nd}-\mathrm{Pb}$ isotope analyses of igneous rock samples. J. Mineral. Petrol. Sci. 107 (2), 74-86 (https://www.jstage.jst.go.jp/article/jmps/107/2/107_110520/_pdf).

Münker, C., Wörner, G., Yogodzinski, G.M., Churikova, T., 2004. Behaviour of high field strength elements in subduction zones: constraints from Kamchatka-Aleutian arc lavas. Earth Planet. Sci. Lett. 224 (3-4), 275-293.

Ozerov, A.Y., 2000. The evolution of high-alumina basalts of the Klyuchevskoy volcano, Kamchatka, Russia, based on microprobe analyses of mineral inclusions. J. Volcanol. Geotherm. Res. 95 (1-4), 65-79. 
Pearce, J.A., 1982. Trace element characteristics of lavas from destructive plate boundaries. In: Thorpe, R.S. (Ed.), Andesites: Orogenic Andesites and Related Rocks. Wiley, New York, pp. 525-548.

Pearce, J.A., 1983. Role of the sub-continental lithosphere in magma genesis at active continental margins. In: Hawkesworth, C.J., Norry, M.J. (Eds.), Continental Basalts and Mantle Xenoliths. Shiva, Nantwich, pp. 230-249.

Piip, B.I., 1946. The activity of the Tolbachik volcano (January 1941). Biulleten vulkanologicheskoi stantsii na Kamchatke 12 pp. 70-73 (In Russian).

Piip, B.I., 1954. Tolbachik volcano. Biull. Vulkanol. Stantsii 20, 69-71 (In Russian).

Pineau, F., Semet, M.P., Grassineau, N., Okrugin, V.M., Javoy, M., 1999. The genesis of the stable isotope $(\mathrm{O}, \mathrm{H})$ record in arc magmas: the Kamtchatka's case. Chem. Geol. 153 (1-4), 93-124.

Ponomareva, V.V., Melekestsev, I.V., Dirksen, O.V., 2006. Sector collapses and large landslides on Late Pleistocene-Holocene volcanoes in Kamchatka, Russia. J. Volcanol. Geotherm. Res. 158 (1-2), 117-138.

Popkov, V.F., 1946. Volcanic activity of Plosky Tolbachik in 1940. Biulleten vulkanologicheskoi stantsii na Kamchatke 12 pp. 54-63 (In Russian).

Portnyagin, M., Hoernle, K., Plechov, P., Mironov, N., Khubunaya, S., 2007a. Constraints on mantle melting and composition and nature of slab components in volcanic arcs from volatiles $\left(\mathrm{H}_{2} \mathrm{O}, \mathrm{S}, \mathrm{Cl}, \mathrm{F}\right)$ and trace elements in melt inclusions from the Kamchatka Arc. Earth Planet. Sci. Lett. 255 (1-2), 53-69.

Portnyagin, M., Bindeman, I., Hoernle, K., Hauff, F., 2007b. Geochemistry of primitive lavas of the Central Kamchatka Depression: magma generation at the edge of the Pacific Plate. In: Eichelberger, J., Gordeev, E., Izbekov, P., Kasahara, M., Lees, J. (Eds.), Volcanism and subduction: the Kamchatka region. Geophysical Monograph Series 172 pp. 199-239.

Reiche, P., 1937. The toreva-block - a distinctive landslide type. J. Geol. 45 (5), 538-548

Romanchev, B.P., Flerov, G.B., 1980. The temperatures of lavas of Grate Fissure Tolbachik Eruption 1975-1976 (Kamchatka), Geokhimiia 5, 688-697 (In Russian).

Saunders, A.D., Norry, M.J., Tarney, J., 1988. Origin of MORB and geochemically-depleted mantle reservoirs: trace element constraints. J. Petrol. Spec. 1, 415-445. http://dx. doi.org/10.1093/petrology/Special_Volume.1.415.

Sigvaldson, G.E., Annertz, K., Nilsson, M., 1992. Effect of glacier loading/deloading on volcanism: postglacial volcanic eruption rate of the Dyngjufjoll area, central Iceland. Bull. Volcanol. 54 (5), 385-392.
Sirin, A.N., Farberov, A.I., 1963. The eruption of Plosky Tolbachik volcano in 1961/62. Biull. Vulkanol. Stantsii 34, 8-11 (In Russian).

Sun, S.S., McDonough, W.F., 1989. Chemical and isotopic systematics of oceanic basalts; implications for mantle composition and processes. In: Saunders, A.D., Norry, M.J. (Eds.), Magmatism in the Ocean Basins. Geological Society of London, London, pp. 313-345.

Takahashi, T., Hirahara, Y., Miyazaki, T., Vaglarov, B.S., Chang, Q., Kimura, J.-I., Tatsumi, Y., 2009. Precise determination of $\mathrm{Sr}$ isotope ratios in igneous rock samples and application to micro-analysis of plagioclase phenocrysts. JAMSTEC Report of Research and Development Special Issue: IFREE Technical Report, pp. 59-64 (http://www.godac. jamstec.go.jp/catalog/data/doc_catalog/media/JAM_RandDsp_08.pdf).

Tatsumi, Y., Kogiso, T., Nohda, S., 1995. Formation of a third volcanic chain in Kamchatka: generation of unusual subduction-related magmas. Contrib. Mineral. Petrol. 120 (2), 117-128.

Timerbaeva, K.M., 1967. Petrology of Klyuchevskoy Volcanoes in Kamchatka. Nauka, Moscow (209 pp. (In Russian). http://www.kscnet.ru/ivs/bibl/srab/timerbaeva.djvu).

Turner, S., McDermott, F., Hawkesworth, C., Kepezhinskas, P., 1998. A U-series study of lavas from Kamchatka and the Aleutians: constraints on source composition and melting processes. Contrib. Mineral. Petrol. 133 (3), 217-234.

Turner, S., Sims, K.W.W., Reagan, M., Cook, C., 2007. A ${ }^{210} \mathrm{~Pb}-{ }^{226}{ }^{R a}-{ }^{230}{ }^{2}{ }^{2}-{ }^{238} \mathrm{U}$ study of Klyuchevskoy and Bezymianny volcanoes, Kamchatka. Geochim. Cosmochim. Acta 71 (19), 4771-4785.

Vinogradov, V.I. Grigoriev, V.S. Leites, A.M., 1988. Age of the metamorphism of the rocks of the Kamchatka Sredinny Range. Izv. Akad. Nauk. SSSR Ser. Geogr. 9, 30-38 (In Russian).

Vinogradov, V.I., Grigoriev, V.S., Kastrykina, V.M., 1991. Age of the metamorphic rocks of the Kamchatka basement. Soviet Geol. 7, 58-65 (In Russian).

Vlodavets, V.I., 1937. Visit the crater of the volcano Plosky Tolbachik on August 13, 1936. Biulleten vulkanologicheskoi stantsii na Kamchatke 2 pp. 27-34 (In Russian).

Volynets, O.N., Babanskii, A.D., Gol'tsman, Y.V., 2000. Variations in isotopic and traceelement composition of lavas from volcanoes of the Northern Group, Kamchatka, in relation to specific features of subduction. Geochem. Int. 38 (10), 974-989.

Volynets, A.O., Melnikov, D.V., Yakushev, A.I., 2013. First data on composition of the volcanic rocks of the IVS 50th anniversary Fissure Tolbachik eruption (Kamchatka). Dokl. Earth Sci. 452 (1), 953-957. http://dx.doi.org/10.1134/S1028334X13090201. 